\title{
1 The evolutionary history of Neandertal and Denisovan 2 Y chromosomes
}

3

4 Martin Petr $^{1 *}$, Mateja Hajdinjak ${ }^{1,13}$, Qiaomei $\mathrm{Fu}^{2,3,4}$, Elena Essel ${ }^{1}$, Hélène Rougier ${ }^{5}$, Isabelle

5 Crevecoeur $^{6}$, Patrick Semal ${ }^{7}$, Liubov V. Golovanova ${ }^{8}$, Vladimir B. Doronichev $^{8}$, Carles Lalueza-

6 Fox $^{9}$, Marco de la Rasilla ${ }^{10}$, Antonio $\operatorname{Rosas}^{11}$, Michael V. Shunkov ${ }^{12}$, Maxim B. Kozlikin ${ }^{12}$,

7 Anatoli P. Derevianko ${ }^{12}$, Benjamin Vernot ${ }^{1}$, Matthias Meyer ${ }^{1}$, Janet Kelso $^{1 *}$

${ }^{1}$ Department of Evolutionary Genetics, Max Planck Institute for Evolutionary Anthropology, D-04103 Leipzig, Germany.

${ }^{2}$ Key Laboratory of Vertebrate Evolution and Human Origins of Chinese Academy of Sciences, IVPP, CAS, Beijing 100044, China.

${ }^{3}$ CAS Center for Excellence in Life and Paleoenvironment, Beijing 100044, China.

${ }^{4}$ University of Chinese Academy of Sciences, Beijing 100049, China.

${ }^{5}$ Department of Anthropology, California State University Northridge, Northridge, California 91330-8244, USA.

${ }^{6}$ Université de Bordeaux, CNRS, UMR 5199-PACEA, 33615 Pessac Cedex, France.

${ }^{7}$ Royal Belgian Institute of Natural Sciences, 1000 Brussels, Belgium.

${ }^{8}$ ANO Laboratory of Prehistory 14 Linia 3-11, St Petersburg 1990 34, Russia.

${ }^{9}$ Institute of Evolutionary Biology, Consejo Superior de Investigaciones Científicas, Universitat Pompeu Fabra, 08003 Barcelona, Spain.

10 Área de Prehistoria, Departamento de Historia, Universidad de Oviedo, 33011 Oviedo, Spain.

${ }^{11}$ Departamento de Paleobiología, Museo Nacional de Ciencias Naturales, Consejo Superior de Investigaciones Científicas, 28006 Madrid, Spain.

12 Institute of Archeology and Ethnography, Siberian Branch, Russian Academy of Sciences, Novosibirsk, Russia

13 The Francis Crick Institute, NW1 1AT London, United Kingdom.

* Correspondence should be addressed to: mp@bodkan.net or kelso@eva.mpg.de 


\section{Abstract}

28 Ancient DNA has allowed the study of various aspects of human history in unprecedented detail.

29 However, because the majority of archaic human specimens preserved well enough for genome

30 sequencing have been female, comprehensive studies of $\mathrm{Y}$ chromosomes of Denisovans and

31 Neandertals have not yet been possible. Here we present sequences of the first Denisovan Y

32 chromosomes (Denisova 4 and Denisova 8), as well as the Y chromosomes of three late

33 Neandertals (Spy 94a, Mezmaiskaya 2 and El Sidrón 1253). We find that the Denisovan Y

34 chromosomes split around 700 thousand years ago (kya) from a lineage shared by Neandertal and

35 modern human $\mathrm{Y}$ chromosomes, which diverged from each other around 370 kya. The

36 phylogenetic relationships of archaic and modern human Y chromosomes therefore differ from

37 population relationships inferred from their autosomal genomes, and mirror the relationships

38 observed on the level of mitochondrial DNA. This provides strong evidence that gene flow from

39 an early lineage related to modern humans resulted in the replacement of both the mitochondrial

40 and Y chromosomal gene pools in late Neandertals. Although unlikely under neutrality, we show

41 that this replacement is plausible if the low effective population size of Neandertals resulted in an

42 increased genetic load in their $\mathrm{Y}$ chromosomes and mitochondrial DNA relative to modern

43 humans. 


\section{Introduction}

46

47

48

49

50

51

52

53

54

Ancient DNA (aDNA) has transformed our understanding of human evolutionary history, revealing complex patterns of population migration, turnover and gene flow, including admixture from archaic humans into modern humans around 55 thousand years ago (kya) (1-4). The majority of insights into the relationships between archaic and modern humans have been based on autosomal sequences, which represent a composite of genealogies of any individual's ancestors. Although mitochondrial DNA (mtDNA) and Y chromosomes only provide information about single maternal and paternal lineages, they offer a unique perspective on various aspects of population history such as sex-specific migration and other cultural phenomena (5-7). Furthermore, because of their lower effective population size $\left(N_{e}\right)$ compared to autosomal loci, coalescent times of mtDNA and $\mathrm{Y}$ chromosomes sampled from two populations provide an upper bound for the last time they experienced gene flow. In this respect, the mtDNA and autosomal sequences of Neandertals, Denisovans and modern humans have revealed puzzling phylogenetic discrepancies. Their autosomal genomes show that Neandertals and Denisovans are sister groups that split from a common ancestor with modern humans between 550-765 kya (8). In contrast, with the TMRCA with modern humans of 360-468 kya, mtDNAs of Neandertals are more similar to the mtDNAs of modern humans than to those of Denisovans (9). Intriguingly, $\sim 400 \mathrm{ky}$ old early Neandertals from Sima de los Huesos were shown to carry mitochondrial genomes related to Denisovan mtDNAs, which is concordant with the autosomal relationships between these groups of archaic humans $(10,11)$. Based on these results it has been suggested that Neandertals originally carried a Denisovan-like mtDNA which was later completely replaced via gene flow from an early lineage related to modern humans $(9,11)$. 
Y chromosomes of Neandertals and Denisovans could provide an important additional source of information about population splits and gene flow events between archaic and modern humans or populations related to them. However, with the exception of a small amount of

70 Neandertal Y chromosome coding sequence $(118 \mathrm{~kb},(12))$, none of the male Neandertals or

71 Denisovans studied to date have yielded sufficient amounts of endogenous DNA to allow

72 comprehensive studies of entire Y chromosomes.

\section{Specimens, DNA capture and genotyping}

74 Previous genetic studies identified two male Denisovans, Denisova 4 (55-84 ky old) and Denisova

758 (106-136 ky old) $(13,14)$, and two male late Neandertals, Spy 94a (38-39 ky old) and

76 Mezmaiskaya 2 (43-45 ky old) (15) (Figure 1A). To enrich for hominin Y chromosome DNA of

77 these individuals, we designed DNA capture probes targeting $\sim 6.9 \mathrm{Mb}$ of the non-recombining

78 portion of the human Y chromosome sequence (Figure 1C, Supplementary Information). Using

79 these probes, we performed hybridization capture on selected single-stranded DNA libraries from

80 Denisova 4, Denisova 8, Spy 94a, and Mezmaiskaya 2 (Supplementary Information). The captured

81 DNA molecules were sequenced from both ends, overlapping reads were merged and aligned to

82 the human reference genome (hg19/GRCh37) (Supplementary Information). Reads of at least 35

83 base-pair (bp) in length that aligned uniquely to the capture target regions were retained for further

84 analysis.

Of the total $\sim 6.9 \mathrm{Mb}$ of $\mathrm{Y}$ chromosome capture target regions, we generated $1.4 \mathrm{X}$ for

87 Table S4.1). In addition, we sequenced 7.9X coverage of a smaller subset of the Y chromosome

88 of the $46-53$-ky-old El Sidrón 1253 Neandertal from Spain (Figure 1B, Table S4.1) (16) by

89 capturing a set of previously generated double-stranded, UDG-treated libraries (17) using a $~ 560$ 
$90 \mathrm{~kb}$ capture array designed to study modern human Y chromosome variation (5). This data provides

91 an opportunity for validating our results with different sample preparation and capture strategies.

To call genotypes for the captured archaic human Y chromosomes, we leveraged the

93 haploid nature of the human Y chromosome and implemented a consensus approach that requires

$9490 \%$ of the reads observed at each site to agree on a single allele, restricting to sites covered by at

95 least three reads (Supplementary information; Table S5.1). This approach minimizes the impact of

96 aDNA damage on genotyping accuracy (Figure S5.1) while allowing for a small proportion of

97 sequencing errors, contamination or misalignment (Supplementary Information). To obtain a

98 reference panel of modern human Y chromosomes for the analyses below, we applied the same

99 genotype calling procedure to a set of previously published modern human $\mathrm{Y}$ chromosomes $(6$,

$10018,19)$ (Supplementary Information).

\section{Archaic Y chromosome phylogeny}

102 To determine the relationships between Denisovan, Neandertal and modern human Y

103 chromosomes we constructed a neighbor-joining tree from the alignment of Y chromosome 104 genotype calls (Supplementary Information). Unlike the rest of the nuclear genome, which puts

105 Denisovans and Neandertals as sister groups to modern humans (2), we found that the Denisovan

106 Y chromosomes form a separate lineage that split before Neandertal and modern human Y 107 chromosomes diverged from each other (Figure 2A, 100\% bootstrap support for both ancestral 108 nodes). Notably, all three late Neandertal Y chromosomes cluster together and fall outside of the 109 variation of present-day human Y chromosomes (Figure 2A, 100\% bootstrap support), which 110 includes the African Y chromosome lineage A00 known to have diverged from all other present111 day human Y chromosomes around 250 kya (6). 


\section{Ages of Y chromosomal ancestors}

113 To estimate the time to the most recent common ancestor (TMRCA) of archaic and modern human

114 Y chromosomes we followed an approach similar to that taken by Mendez et al., expressing this

115 TMRCA relative to the deepest known split within present-human Y chromosomes (African Y

116 chromosome lineage A00, $(6,12)$, Supplementary Information). This has the advantage of not

117 relying on private mutations on the archaic human branch which makes it robust to low coverage

118 and aDNA damage which vary significantly between samples (Figure 2A, Table S4.1, Figure 119 S5.1).

We first calculated the mutation rate in the total $6.9 \mathrm{Mb}$ target regions to be $7.34 \times 10^{-10}$ per

121 bp per year (bootstrap CI: 6.27-8.46 $\times 10^{-10}$; Figure S7.1, Table S7.2, Supplementary Information).

122 Using this mutation rate, we estimated the TMRCA of the African A00 lineage and a set of non-

123 African Y chromosomes from the SGDP panel $(6,19)$ at 249 kya (bootstrap CI: 213-293 kya;

124 Figure S7.1, Table S7.2, Supplementary information). These estimates are consistent with values

125 inferred from larger-scale studies of present-day human Y chromosomes $(6,18)$, suggesting that

126 the Y chromosomal regions we defined for capture are not unusual in terms of their mutation rate.

127 Second, assuming the A00 divergence time of 249 kya, we inferred TMRCAs between

128 archaic $\mathrm{Y}$ chromosomes and present-day non-African $\mathrm{Y}$ chromosomes for each archaic individual

129 at a time (Figure S7.4, Table S7.3, Supplementary information). We found that the two Denisovan

130 Y chromosomes (Denisova 4 and Denisova 8) split from the modern human lineage around 700

131 kya (CI: 607-833 kya for the higher coverage Denisova 8, Figure 2B, Table S7.3). In contrast, the

132 three Neandertal Y chromosomes split from the modern human lineage about 350 kya: 353 kya

133 for Spy $94 a$ (286-449 kya), 369 kya for Mezmaiskaya 2 (326-419 kya) and 339 kya for El Sidrón

$134 \quad 1253$ (275-408 kya) (Figure 2B, Table S7.3). Additionally, we used the proportions of sharing of 
derived alleles with the high-coverage Mezmaiskaya 2 Y chromosome to estimate the TMRCA of the three Neanderthal Y chromosomes at around 100 kya (Figure S7.14 and S7.15). We validated the robustness of all TMRCA estimates by repeating the analyses using filters of varying levels of

138 stringency and different genotype calling methods (Figures S7.9, S7.11, S7.13). Similarly, 139 although we detected some evidence of capture bias in the data (Figure S4.5), we observed no 140 significant differences between capture data and shotgun sequences or between individuals 141 showing different read length distributions, indicating that the effect of technical biases on our 142 inferences is negligible (Figure S7.11).

The Denisovan-modern human Y chromosome TMRCA estimates are in good agreement

144 with population split times inferred from autosomal sequences, suggesting that the differentiation

145 of Denisovan Y chromosomes from modern humans occurred through a simple population split

146 (20). In contrast, the Neandertal-modern human Y chromosome TMRCAs are significantly

147 younger than the inferred population split time (Figure 3A) and consistent with a time window for 148 gene flow from a lineage related modern humans into Neandertals inferred from mtDNA (9) and 149 autosomal sequences $(21,22)$.

\section{Disagreement with the previous Y chromosome TMRCA}

151 Our estimates of the Neandertal-modern human TMRCA, including those obtained using the larger 152 amount of data we generated from the same individual (TMRCA of 339 kya, Figure 2B), are 153 substantially younger than the previous estimate of 588 kya from the El Sidrón 1253 Neandertal 154 (12). The previous estimate was based on $\sim 3 \mathrm{X}$ coverage of $118 \mathrm{~kb}$ of exome capture sequence and, 155 due to the limited amount of data, used SNPs supported even by single reads $(12,17)$. Although 156 the TMRCA inference procedure used by (12) does not rely directly on the counts of private 157 mutations on the archaic lineage (Figure S7.4, Supplementary Information), it can still be affected 
by erroneous genotype calls, which can lead to shared derived variants being converted to the ancestral state, increasing the apparent TMRCA. Indeed, when we applied our stricter filtering criteria to the El Sidrón 1253 data analyzed previously, we arrived at TMRCA estimates for El

Sidrón 1253 that are consistent with the other Neandertals in our study (Figure S7.12).

The phylogenetic relationships of archaic and modern human $\mathrm{Y}$ chromosomes are similar to the observations made from mtDNA genomes $(9,10)$, suggesting that both mtDNA and $\mathrm{Y}$ chromosomes of early Neandertals have been replaced via gene flow from an early lineage related to modern humans, possibly as a result of the same population contact (Figure 3A, (9)). Although such contact has been proposed, previous work suggests gene flow from modern humans into Neandertals on the order of only a few percent $(21,23)$. Assuming neutrality, the fixation probability of a locus is equal to its initial frequency in a population (24). Therefore, the joint probability of both Neandertal mtDNA and Y chromosome replacements by their modern human counterparts under neutrality is even lower. However, several studies have suggested that due to

172 their low $N_{e}$ and reduced efficacy of purifying selection, Neandertals may have accumulated an 173 excess of deleterious variation compared to modern humans $(25,26)$ and sequencing of the exomes

174 of three Neandertals directly demonstrated that they carried more deleterious alleles than present175 day humans (17). To explore the dynamics of introgression into Neandertals, we simulated the 176 frequency trajectories of a non-recombining, uniparental locus under a model of purifying 177 selection (Supplementary Information, (27)). We simulated lower $N_{e}$ on the Neandertal lineage 178 after its split from modern humans and accumulation of deleterious variants on both lineages 179 across a grid of several relevant parameters (such as the time of the split between both populations 180 or the amount of sequence under negative selection). For each combination of parameters, we then 
181 calculated the ratio of fitnesses of average Neandertal and average modern Y chromosomes

182 produced by the simulation, and traced the trajectory of introgressed modern human $\mathrm{Y}$

183 chromosomes in Neandertals over 100 ky following 5\% admixture (Figures S8.1 and S8.2,

184 Supplementary information).

We found that even a small reduction in fitness of Neandertal Y chromosomes compared to modern human $\mathrm{Y}$ chromosomes has a strong effect on the probability of a complete replacement by introgressed modern human Y chromosomes (Figure 3B). Specifically, even a 1\% reduction in

188 Neandertal Y chromosome fitness increases the probability of replacement after $20 \mathrm{ky}$ to $\sim 25 \%$ and 189 a $2 \%$ reduction in fitness increases this probability to $\sim 50 \%$. This fitness reduction measure is an 190 aggregate over all linked deleterious mutations on the $\mathrm{Y}$ chromosome and integrates a number of 191 biological parameters, only a subset of which we consider here (Figure S8.1 and S8.3).

192 Importantly, we note that although we simulated introgression of Y chromosomes, the abstract 193 measure of fitness reduction of a non-recombining, uniparental locus can also be generalized to 194 the case of mtDNA introgression (Figure 3B).

These results show that a model of higher genetic load in Neandertals is compatible with 196 an increased probability of replacement of Neandertal mtDNA and Y chromosomes with their 197 introgressed modern human counterparts. Furthermore, given the crucial role of the Y 198 chromosome in reproduction and fertility, and its haploid nature, it is possible that deleterious 199 mutations or structural variants on the Y chromosome have a dramatically larger impact on fitness 200 than we considered in our simulations (28).

\section{Conclusions}

202 Our results show that the Y chromosomes of late Neandertals represent an extinct lineage related 203 to modern human Y chromosomes that introgressed into Neandertals some time between $\sim 370$ kya 
204 and $\sim 100$ kya. The presence of this Y chromosome lineage in all late Neandertals makes it unlikely

205 that genetic changes that accumulated in Neandertal and modern human Y chromosomes prior to

206 the introgression lead to incompatibilities between these groups of humans. We predict that the

$207 \sim 400$ ky old Sima de los Huesos individuals, who are early Neandertals but carry a Denisovan-like

208 mtDNA $(10,11)$, should also carry a Y chromosome lineage more similar to Denisovans than to

209 later Neandertals. Although complete replacement of mtDNA and Y chromosomes might seem

210 surprising given that limited modern human gene flow has been detected in the genomes of late

211 Neandertals $(15,21-23)$, mitochondrial-autosomal discrepancies are predicted by population

212 genetic theory, and are relatively common during interspecific hybridization in the animal

213 kingdom (29-31). Our simulations show that differences in genetic loads in uniparental loci

214 between the two hybridizing populations is a plausible driver of this phenomenon. 


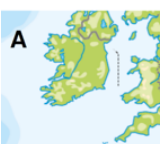

(E)

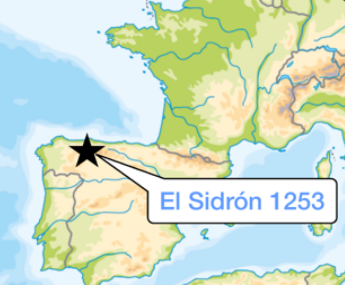

(Spy $94 a$
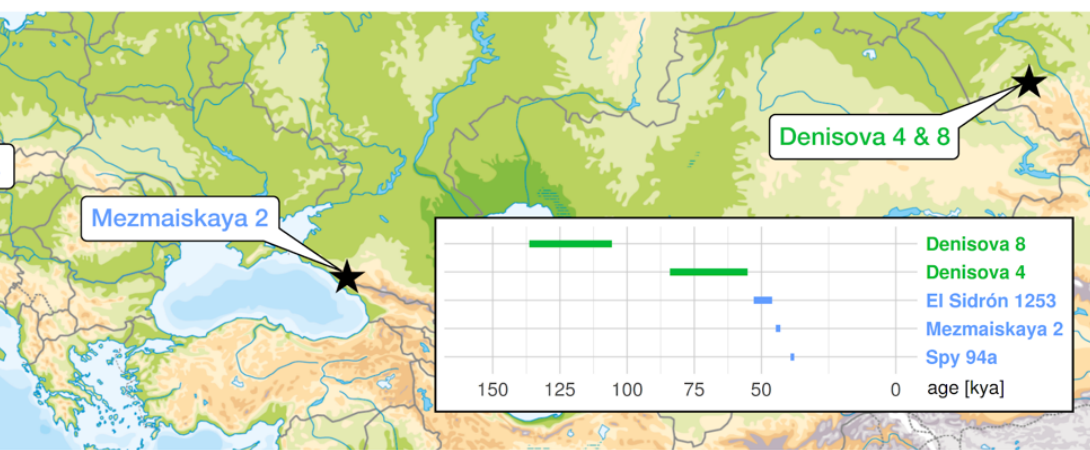

B

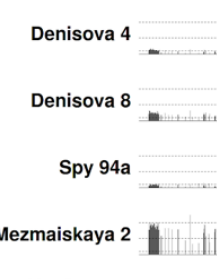

El Sidrón 1253

$(560 \mathrm{~kb})$

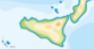

$\begin{array}{lllll}150 & 125 & 100 & 75 & 50\end{array}$

0 age [kya]

C

Figure 1. Geographical locations, ages and sequencing coverage of the male archaic humans

in our study. (A) Locations of archaeological sites where the five archaic human specimens have sequencing coverage for each archaic human $\mathrm{Y}$ chromosome along the $\sim 6.9 \mathrm{Mb}$ of capture target regions. The heights of the thin vertical bars represent average coverage in each target region. The

224 chromosomal coordinates are aligned to match the $\mathrm{Y}$ chromosome structure depicted in panel C.

225 Right - Distribution of coverage across all target sites for each archaic Y chromosome on the left.

226 (C) Genomic structure of the portion of the human Y chromosome targeted for capture. Thin black

227 vertical lines show the position of individual target capture regions. The coordinates of $\mathrm{Y}$

228 chromosome regions were taken from (32). 
A

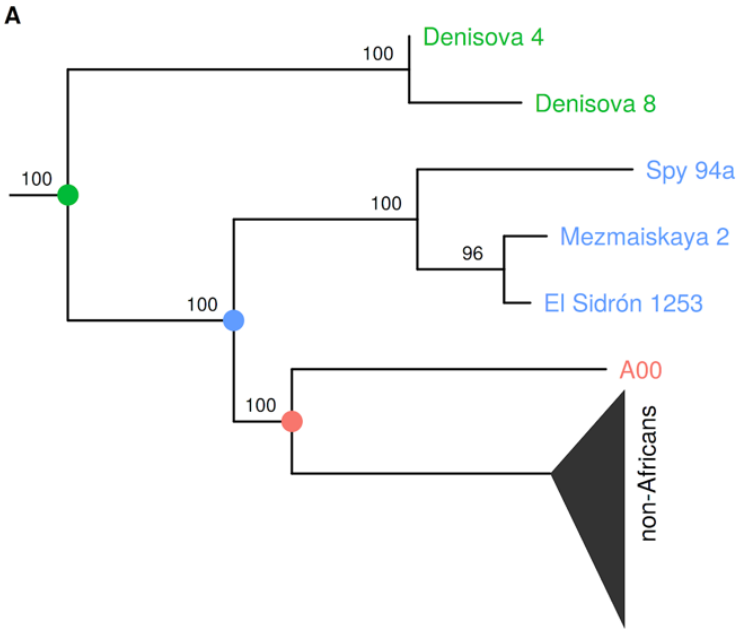

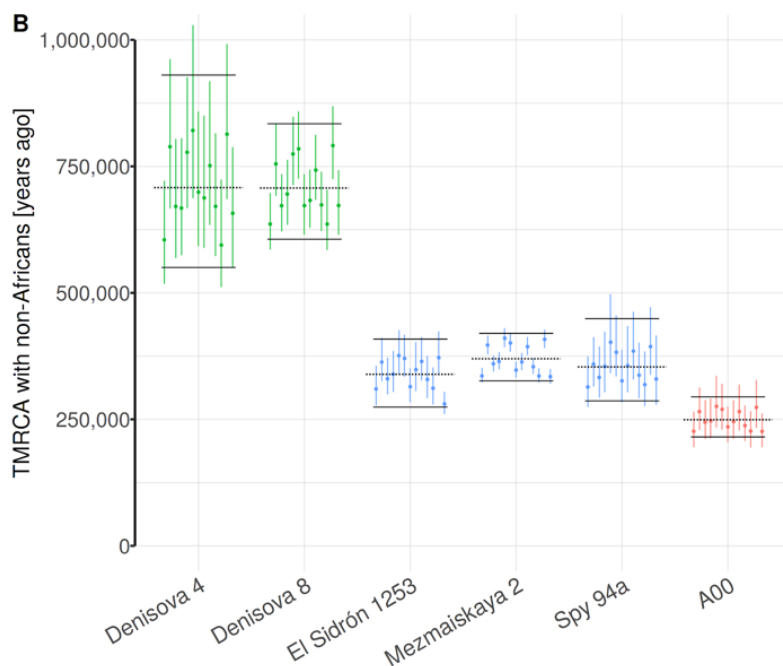

Figure 2. Phylogenetic relationships between archaic and modern human Y chromosomes.

(A) Neighbor-joining tree based on the alignment of Y chromosome genotype calls, excluding Cto-T and G-to-A polymorphisms to mitigate the effects of aDNA damage. Numbers next to the internal nodes show bootstrap support for the three major clades (green - Denisovans, blue Neandertals, red - deeply divergent African lineage A00) based on 100 bootstrap replicates. The

237 tree was rooted using a chimpanzee Y chromosome as the outgroup. We note that the terminal

238 branch lengths are not informative about the ages of specimens due to differences in sequence 239 quality (Figure 1A). (B) Distributions of the times to the most recent common ancestor (TMRCA)

240 between $\mathrm{Y}$ chromosomes listed along the x-axis and a panel of 13 non-African Y chromosomes.

241 Each dot represents a TMRCA estimate based on a single non-African Y chromosome, with error

242 bars showing approximate 95\% C.I. based on resampling of branch counts (Supplementary

243 Information). Black horizontal lines show the mean TMRCA calculated across the full non-African 244 panel (dotted lines) with bootstrap-based 95\% C.I. (solid lines). 

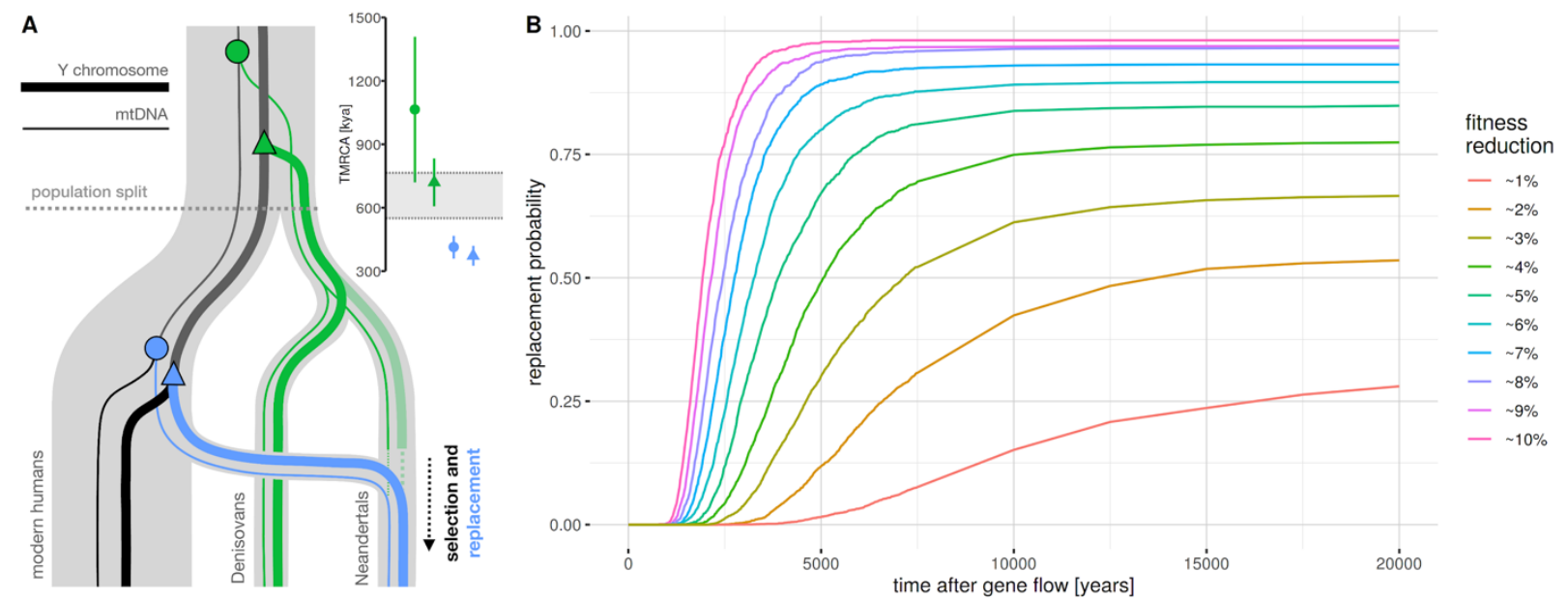

Figure 3. Proposed model for the replacement of Neandertal Y chromosomes and mtDNA.

(A) Schematic representation of the relationships between archaic and modern human mtDNA

(thin lines) and Y chromosomes (thick lines) based on current phylogenetic inferences, with the

yet unsampled, hypothetical Y chromosome which was replaced by an early lineage related to

modern human Y chromosomes. Positions of relevant most recent common ancestors with modern shows TMRCA estimates for the four nodes in the diagram: Y chromosome TMRCAs as estimated by our study, mtDNA TMRCAs estimates from the literature $(9,10)$. The grey horizontal bar highlights the $95 \%$ C.I. for the population split time between archaic and modern humans (8). (B) Probability of replacement of a non-recombining, uniparental Neandertal locus as a function of time after gene flow, assuming a given level of fitness burden relative to its modern human counterpart. Trajectories are based on forward simulations across a grid of parameters (Figure pulse at $5 \%$. 
263 1. R. E. Green et al., Science 328, 710 (2010).

264 2. M. Meyer et al., Science 338, 222 (2012).

265 3. I. Lazaridis et al., Nature 513, 409 (2014).

266 4. Q. Fu et al., Nature 534, 200 (2016).

267 5. S. Lippold et al., Investig Genet 5, 13 (2014).

268 6. M. Karmin et al., Genome Research 25, 459 (2015).

269 7. I. Olalde et al., Science 363, 1230 (2019).

270 8. K. Prüfer et al., Nature 505, 43 (2014).

271 9. C. Posth et al., Nat Commun 8, 16046 (2017).

272 10. M. Meyer et al., Nature 505, 403 (2014).

273 11. M. Meyer et al., Nature 531, 504 (2016).

274 12. F. Mendez, G. Poznik, S. Castellano, C. Bustamante, The American Journal of Human

275 Genetics 98, 728 (2016).

276 13. S. Sawyer et al., Proc Natl Acad Sci U S A 112, 15696 (2015).

277 14. K. Douka et al., Nature 565, 640 (2019).

278 15. M. Hajdinjak et al., Nature 555, 652 (2018).

279 16. R. E. Wood et al., Archaeometry 55, 148 (2013).

280 17. S. Castellano et al., Proceedings of the National Academy of Sciences 111, 6666 (2014).

281 18. Q. Fu et al., Nature 514, 445 (2014).

282 19. S. Mallick et al., Nature 538, 201 (2016).

283 20. K. Prüfer et al., Science 358, 655 (2017).

284 21. M. J. Hubisz, A. L. Williams, A. Siepel, (2019).

285 22. L. Chen, A. B. Wolf, W. Fu, L. Li, J. M. Akey, Cell 180, 677 (2020).

286 23. M. Kuhlwilm et al., Nature 530, 429 (2016).

287 24. J. F. Crow, M. Kimura, An Introduction to Population Genetics Theory 1970), pp. 591.

288 25. K. Harris, R. Nielsen, Genetics 203, 881 (2016).

289 26. I. Juric, S. Aeschbacher, G. Coop, PLoS Genet 12, e1006340 (2016).

290 27. B. C. Haller, P. W. Messer, Molecular biology and evolution 36, 632 (2019).

291 28. S. Colaco, D. Modi, Reproductive biology and endocrinology 16, 14 (2018).

292 29. J. W. Ballard, M. C. Whitlock, Mol Ecol 13, 729 (2004).

293 30. T. Bonnet, R. Leblois, F. Rousset, P.-A. Crochet, Evolution 71, 2140 (2017).

294 31. F. A. Seixas, P. Boursot, J. Melo-Ferreira, Genome Biol 19, 91 (2018).

295

296

297

\section{Acknowledgements}

299 We would like to thank Svante Pääbo, Mark Stoneking, Benjamin Peter, Montgomery Slatkin,

300 Laurits Skov and Elena Zavala for helpful discussions and comments on the manuscript. Q.F. was

301 supported by funding from the Chinese Academy of Sciences (XDB26000000), and the National

302 Natural Science Foundation of China (91731303, 41925009,41630102). A.R. was funded by 
304 the Spy collection by H.R., I.C. and P.S. was supported by the Belgian Science Policy Office

305 (BELSPO 2004-2007, MO/36/0112). M.S., M.K. and A.D. were supported by the Russian 306 Foundation for Basic Research (RFBR 17-29-04206). This study was funded by the Max Planck 307 Society and the European Research Council (grant agreement number 694707). M.P., J.K. 308 analyzed data. M.H., Q.F., E.E. performed laboratory experiments. H.R., I.C., P.S., L.V.G., 309 V.B.D., C.L.-F., M.d.1.R., A.R., M.V.S., M.B.K., A.P.D. provided samples. B.V., M.M., J.K. 310 supervised the project. M.P., J.K. wrote and edited the manuscript with input from all co-authors.

312 Data and materials availability: All sequence data are available from the European Nucleotide 313 Archive under accession numbers xxx (Mezmaiskaya 2), xxx (Spy 94a), xxx (Denisova 4), xxx 314 (Denisova 8) and xxx (El Sidrón 1253).

316 Complete source code for data processing and simulations, as well as Jupyter notebooks with all 317 analyses and results can be found at https://github.com/bodkan/archaic-ychr. All data is available 318 from https://bioinf.eva.mpg.de/archaic-ychr. 


\section{Supplementary Information}

11 Martin Petr, Mateja Hajdinjak, Qiaomei Fu, Elena Essel, Hélène Rougier, Isabelle

12 Crevecoeur, Patrick Semal, Liubov V. Golovanova, Vladimir B. Doronichev, Carles

13 Lalueza-Fox, Marco de la Rasilla, Antonio Rosas, Michael V. Shunkov, Maxim B. Kozlikin,

14 Anatoli P. Derevianko, Benjamin Vernot, Matthias Meyer, Janet Kelso 
17 Complete source code for data processing and simulations, as well as Jupyter notebooks 18 with all analyses can be found at https://github.com/bodkan/archaic-ychr. All data is 19 available from https://bioinf.eva.mpg.de/archaic-ychr.

3. Sequencing and data processing

4. Coverage and measures of ancient DNA quality

4.2. Patterns of ancient DNA damage

4.3. Read length distribution

4.4. Modern human contamination

5.1. Consensus genotype calling

7. Estimating the TMRCA of archaic and modern human $\mathrm{Y}$ chromosomes

7.1. TMRCA of Africans and non-Africans $T M R C A_{A F R}$ 


\section{Y chromosome DNA capture design}

47 To design a set of DNA capture probes, we identified regions of the human $Y$

48 chromosome that are uniquely mappable with short sequence reads. Starting from the

49 entire human $\mathrm{Y}$ chromosome reference sequence (version hg19), we removed regions

50 that overlap those found by the Tandem Repeats Finder (1) and those identified by a

51 previously described mappability track as regions that may result in ambiguous alignment

52 of short reads (so called "map35_50\%" filter, (2)). We then removed any regions that were

53 shorter than $99 \mathrm{bp}$ of continuous sequence. In total, this process yielded 6,912,728 bp

54 ( 6.9 Mb) of the $\mathrm{Y}$ chromosome suitable for use as an ancient DNA capture target.

$55 \quad$ We designed 52 bp oligonucleotide probes by tiling the identified $6.9 \mathrm{Mb}$ of target

56 sequence with $52 \mathrm{bp}$ fragments in steps of $3 \mathrm{bp}$. This resulted in 2,049,846 individual

57 oligonucleotide probes. To verify that the probe sequences are unique genome-wide, we

58 aligned each probe to the complete hg19 reference sequence and confirmed that they all

59 aligned only to their expected position on the $Y$ chromosome with mapping quality of at

60 least 30 . The files containing the coordinates of target regions, as well as the coordinates

61 and sequences of all capture probes, including 8 bp adapters, are freely available from

62 https://bioinf.eva.mpg.de/archaic-ychr.

64 Following the approach taken by Fu et al. (3), 60 bp oligonucleotides containing the probe 65 sequences as well as an 8 bp universal linker sequence were synthesized on three One 66 Million Feature Arrays (Agilent Technologies), converted into probe libraries and 67 amplified. Single-stranded biotinylated DNA probes were generated using a linear 68 amplification reaction with a single biotinylated primer (3). 
70 We also co-analyzed data from two additional captures carried out previously: (i) 120 kb

71 of $Y$ chromosome sequence from the El Sidrón 1253 Neandertal that was targeted as a

72 part of an exome capture study (4) and has been analyzed previously (5), and (ii) a larger

73 amount of data ( $560 \mathrm{~kb})$ from the same El Sidrón 1253 individual which we captured

74 using probes designed for a previously published set of $Y$ chromosome target regions (6).

75 The file containing the coordinates of target regions and coordinates and sequences of

76 all capture probes, including 8 bp adapters, is freely available from

77 http://bioinf.eva.mpg.de/archaic-ychr.

78

79 The features of our new capture design, as well as a comparison with the $Y$ chromosome

80 target regions on the exome capture $(4,5)$ and the $\sim 560 \mathrm{~kb}$ capture $(6)$ are reported in

\section{Table S1.1.}

82 


\begin{tabular}{|l|l|l|l|l|l|l|}
\hline \multicolumn{1}{|c|}{ target } & total [bp] & \# of regions & $\begin{array}{c}\text { min } \\
{[\text { [bp] }}\end{array}$ & $\begin{array}{c}\text { median } \\
{[\text { [bp] }}\end{array}$ & $\begin{array}{c}\text { mean } \\
{[\text { bp] }}\end{array}$ & $\begin{array}{c}\text { max } \\
{[\text { [bp] }}\end{array}$ \\
\hline entire mappable $Y$ & $6,912,728$ & 15,903 & 99.0 & 240.0 & 434.7 & $9,425.0$ \\
\hline$\sim 560$ kb capture $\left(^{*}\right)$ & 573,657 & 1,251 & 60.0 & 151.0 & 458.6 & 3899.0 \\
\hline$\sim 560$ kb capture & 556,259 & 1,779 & 1.0 & 119.0 & 312.7 & $2,829.0$ \\
\hline exome subset & 118,643 & 2,519 & 1.0 & 3.0 & 47.1 & $1,257.0$ \\
\hline
\end{tabular}

86 Table S1.1. Characteristics of the three sets of $Y$ chromosome capture targets

87 analyzed in our study. "Exome subset" refers to a $Y$ chromosome subset of the exome

88 capture sequence generated by Castellano et al. and analyzed by Mendez et al. (called

89 "filter 1") $(4,5)$, “ 560 kb capture” refers to target regions originally designed for studying

90 present-day human $\mathrm{Y}$ chromosome variation (6), star $\left(^{*}\right)$ signifies statistics before

91 intersecting the original set of target regions with the "map35_50\%" filter (2), "entire

92 mappable $Y^{\prime \prime}$ represents capture regions targeting the entire mappable portion of the

93 human Y chromosome designed for our study.

94

95

96 
$97 \quad$ 2. Sampling, DNA extraction, library preparation and capture

98 Samples of $15.4 \mathrm{mg}$ and $14.9 \mathrm{mg}$ of tooth powder from Denisova 8 were used for DNA 99 extraction using a silica-based method (7) with modifications as described in (8). Ten $\mathrm{mg}$ 100 of the tooth powder from Denisova 4 were used for a silica-based DNA extraction that is 101 optimized for the recovery of extremely short DNA fragments (9). Four samples of 102 Mezmaiskaya 2 bone powder, ranging between $3.2 \mathrm{mg}$ and $17.5 \mathrm{mg}$ were treated with $1030.5 \%$ hypochlorite solution to minimize microbial and present-day human DNA 104 contamination (8) before DNA was extracted either manually (7) or on an automated liquid handling platform (Bravo NGS workstation B, Agilent Technologies) (10). See Table S2.1 for an overview of the DNA extracts and libraries generated in this and previous studies and the experimental conditions used. were prepared from DNA extracts made for this study (Table S2.1). Two of the singlestranded DNA libraries for Denisova 8 (A9461 and A9462) were prepared manually using $10 \mu \mathrm{L}$ of each extract as an input (12). All other single-stranded DNA libraries were 114 prepared using either $10 \mu \mathrm{L}$ or $30 \mu \mathrm{L}$ of extract as input (13) on an automated liquid 115 handling platform (Bravo NGS workstation B, Agilent Technologies) (14). All new libraries were prepared without UDG treatment (non-UDG treated libraries). In order to monitor the efficiency of library preparation, a control oligonucleotide was spiked into each aliquot of a DNA extract used for library preparation (9). Quantitative 
120 of oligonucleotides that were successfully converted to library molecules $(9,13)($ Table

121 S2.1). Each library was tagged with two unique index sequences (15) and amplified into

122 plateau with AccuPrime Pfx DNA polymerase (Life Technologies) (16) according to the

123 modifications detailed in (8). Fifty microlitres (half of the total volume) of each of the

124 amplified libraries were purified on an automated liquid handling platform (Bravo NGS

125 workstation B, Agilent Technologies) using SPRI beads (14). A NanoDrop 1000

126 Spectrophotometer (NanoDrop Technologies) was used to determine the concentrations

127 of the purified libraries.

128 In solution hybridization capture of the $\mathrm{Y}$ chromosome was performed in two

129 successive rounds of capture as described previously (3), using the $Y$ chromosome probe

130 set designed in the present study and single-stranded libraries prepared in this and

131 previous studies. In addition, we performed hybridization capture on 40 double-stranded

132 libraries prepared in a previous study from the El Sidrón 1253 Neandertal (see Table S1

133 in (4)) using a smaller $\sim 560 \mathrm{~kb} \mathrm{Y}$ chromosomal probe set that was also designed

134 previously (6). 


\section{Sequencing and data processing}

\subsection{Newly generated archaic human $Y$ chromosomes}

138 All captured libraries were sequenced on the Illumina HiSeq 2500 platform in a double 139 index configuration (2x76 cycles) (15), and base calling was done using Bustard 140 (Illumina). Adapters were trimmed and overlapping paired-end reads were merged using 141 leeHom (17). The Burrows-Wheeler Aligner (BWA) (18) with parameters adjusted for 142 alignment of ancient DNA (“-n $0.01-02$ - 2 16500") was used to align the sequenced 143 fragments to the human reference genome version hg19/GRCh37. Only reads showing 144 perfect matches to the expected index sequence combinations were retained for 145 subsequent analyses. PCR duplicates were removed using the bam-rmdup program, 146 which can be downloaded in source form from https://github.com/mpieva/biohazard-tools.

147 DNA fragments that were at least 35 base pairs (bp) long and had a mapping quality of 148 at least 25 were extracted using samtools (19). Each processed and filtered BAM file (one

149 for each archaic human $Y$ chromosome) was intersected with a BED file of the appropriate $150 \mathrm{Y}$ chromosome target (full $\sim 6.9 \mathrm{Mb}$ capture, $\sim 120 \mathrm{~kb}$ exome capture or $\sim 560 \mathrm{~kb}$ capture).

\subsection{Previously published archaic human sequences}

152 In addition to the new capture data generated here, we analyzed previously published 153 shotgun sequences of the Spy 94a and Mezmaiskaya 2 individuals (11), as well as exome 154 capture data of the El Sidrón 1253 individual (4). For comparisons to our capture data, 155 we generated BAM files for Spy 94a and Mezmaiskaya 2 shotgun sequences and the El 156 Sidrón 1253 exome capture by filtering the published data to minimum read length of 35 
157 bp and mapping quality 25 , keeping only sequences aligned to the set of appropriate 158 target capture regions ( 6.9 Mb capture target for Spy 94a and Mezmaiskaya 2, 118 kb 159 capture target for El Sidrón 1253, Table S1.1).

\subsection{Previously published modern human sequences}

161 For comparisons with modern human $Y$ chromosomes, we downloaded 19 BAM files of 162 African and non-African $Y$ chromosomes published by the Simons Genome Diversity 163 Project (SGDP) (20), two Y chromosomes representing the African A00 lineage (21) and 164 the $Y$ chromosome of a $\sim 45,000$-year-old hunter-gatherer Ust'-Ishim (22). Because the 165 two individuals from which the $\mathrm{A} 00 \mathrm{Y}$ chromosomes were sequenced are closely and 166 each is only about half of the coverage of the other modern human $\mathrm{Y}$ chromosomes 167 (Table S4.3), we followed the approach of the original A00 publication and merged the 168 two A00 Y chromosomes into a single BAM file $(21,23)$. All individual BAM files (one for 169 each modern human $\mathrm{Y}$ chromosome) were then filtered to retain reads with a minimum 170 length of $35 \mathrm{bp}$ and mapping quality of at least 25 , and alignment to the appropriate set 171 of $\mathrm{Y}$ chromosome target capture regions (Table S1.1). 


\section{4. Coverage and measures of ancient DNA quality}

175 Sequencing coverage was calculated using bedtools (24). To get coverage for a given

176 individual in a given set of target regions, we ran the command bedtools coverage -

177 a $\angle B E D>-b<B A M>-d$, which reports the coverage for each position in a BED file in

178 the last column of its output. We removed sites with coverage higher than the $98 \%$

179 quantile of the entire distribution in each of the individuals in our study. Figure 1B (spatial

180 distribution and overall distribution) and Tables S4.1, S4.2 and S4.3 summarise the

181 values of coverage at sites with less than $98 \%$ quantile of the overall distribution in a 182 sample. 


\begin{tabular}{|l|c|l|}
\hline \multicolumn{1}{|c|}{ individual } & $\begin{array}{c}\text { mean } \\
\text { coverage }\end{array}$ & target \\
\hline Spy 94a & 0.8 & $6.9 \mathrm{Mb}$ \\
\hline Denisova 4 & 1.4 & $6.9 \mathrm{Mb}$ \\
\hline Denisova 8 & 3.5 & $6.9 \mathrm{Mb}$ \\
\hline El Sidrón 1253 & 7.9 & $560 \mathrm{~kb}$ \\
\hline Mezmaiskaya 2 & 14.3 & $6.9 \mathrm{Mb}$ \\
\hline
\end{tabular}

185 Table S4.1. Mean coverage of archaic human $\mathrm{Y}$ chromosomes sequenced in this

186 study. Sites with coverage higher than $98 \%$ quantile of the entire distribution were 187 excluded from the calculation. 


\begin{tabular}{|l|c|c|l|}
\hline \multicolumn{1}{|c|}{ name } & mean coverage & target & \multicolumn{1}{c|}{ study } \\
\hline Spy 94a (shotgun) & 0.5 & $6.9 \mathrm{Mb}$ & Hajdinjak et al., 2018 \\
\hline Mezmaiskaya 2 (shotgun) & 0.8 & $6.9 \mathrm{Mb}$ & Hajdinjak et al., 2018 \\
\hline El Sidrón 1253 (capture) & 3.2 & $118 \mathrm{~kb}$ & Castellano et al., 2014, Mendez et al., 2016 \\
\hline
\end{tabular}

190

191 Table S4.2. Mean coverage of previously published archaic human Y chromosome

sequences. The coverage reported for Spy 94a and Mezmaiskaya 2 shotgun sequence

193 data is that of sequences overlapping the 6.9 Mb Y capture regions. The El Sidrón 1253

194 libraries were captured using an exome capture array $(4,5)$ and the coverage reported

195 here is for the $\sim 118 \mathrm{~kb}$ exome target capture regions. For each individual, sites with 196 coverage higher than $98 \%$ quantile of the entire distribution were excluded from the 197 calculation. 


\begin{tabular}{|c|c|c|c|}
\hline name & $\begin{array}{c}\text { mean } \\
\text { coverage }\end{array}$ & target & study \\
\hline A00-1 & 8.8 & $6.9 \mathrm{Mb}$ & Karmin et al., 2015 \\
\hline A00-2 & 12.0 & $6.9 \mathrm{Mb}$ & Karmin et al., 2015 \\
\hline S_Mandenka-1 & 16.3 & $6.9 \mathrm{Mb}$ & Mallick et al., 2016 \\
\hline S_Yoruba-2 & 17.0 & $6.9 \mathrm{Mb}$ & Mallick et al., 2016 \\
\hline S_Finnish-2 & 17.0 & $6.9 \mathrm{Mb}$ & Mallick et al., 2016 \\
\hline S_Punjabi-1 & 17.1 & $6.9 \mathrm{Mb}$ & Mallick et al., 2016 \\
\hline S_Sardinian-1 & 18.1 & $6.9 \mathrm{Mb}$ & Mallick et al., 2016 \\
\hline S_Dai-2 & 19.8 & $6.9 \mathrm{Mb}$ & Mallick et al., 2016 \\
\hline S_Gambian-1 & 20.1 & $6.9 \mathrm{Mb}$ & Mallick et al., 2016 \\
\hline Ust'-Ishim & 20.1 & $6.9 \mathrm{Mb}$ & Fu et al., 2014 \\
\hline S_Mbuti-1 & 20.3 & $6.9 \mathrm{Mb}$ & Mallick et al., 2016 \\
\hline S_Dinka-1 & 20.8 & $6.9 \mathrm{Mb}$ & Mallick et al., 2016 \\
\hline S_Han-2 & 20.8 & $6.9 \mathrm{Mb}$ & Mallick et al., 2016 \\
\hline$A 00$ & 20.9 & $6.9 \mathrm{Mb}$ & merged $\mathrm{A} 00-1$ and $\mathrm{A} 00-2$ \\
\hline S_BedouinB-1 & 21.7 & $6.9 \mathrm{Mb}$ & Mallick et al., 2016 \\
\hline S_French-1 & 21.9 & $6.9 \mathrm{Mb}$ & Mallick et al., 2016 \\
\hline S_Karitiana-1 & 22.2 & $6.9 \mathrm{Mb}$ & Mallick et al., 2016 \\
\hline S_Turkish-1 & 22.5 & $6.9 \mathrm{Mb}$ & Mallick et al., 2016 \\
\hline S_Saami-2 & 22.6 & $6.9 \mathrm{Mb}$ & Mallick et al., 2016 \\
\hline S_Ju_hoan_North-1 & 22.7 & $6.9 \mathrm{Mb}$ & Mallick et al., 2016 \\
\hline S_Papuan-2 & 23.2 & $6.9 \mathrm{Mb}$ & Mallick et al., 2016 \\
\hline S_Thai-1 & 25.1 & $6.9 \mathrm{Mb}$ & Mallick et al., 2016 \\
\hline S_Burmese-1 & 29.2 & $6.9 \mathrm{Mb}$ & Mallick et al., 2016 \\
\hline
\end{tabular}

201 Table S4.3. Mean coverage of modern human Y chromosomes in capture target regions. Coverage is reported using sequences within the $6.9 \mathrm{Mb}$ target capture regions.

203 For each individual, sites with coverage higher than $98 \%$ quantile of the entire distribution 204 were excluded from the calculation. 


\subsection{Patterns of ancient DNA damage}

208 To check for the presence of genuine ancient DNA sequences, we looked for an 209 increased rate of deamination-induced substitutions, an important signature of ancient

210 DNA damage (25). We counted substitution frequencies for each individual BAM file (one

211 BAM file per individual $Y$ chromosome) and found that molecules from single-stranded 212 libraries that were not treated by uracil-DNA glycosylase (UDG) enzyme (those from Spy 213 94a, Mezmaiskaya 2, Denisova 4 and Denisova 8) show highly elevated frequencies of 214 C-to-T substitutions towards the ends of molecules, as well as C-to-T substitutions 215 throughout the molecules (Figure S4.1). As is characteristic of double-stranded libraries 216 treated with the UDG enzyme, the deamination substitution frequency signal in the 217 capture data from El Sidrón 1253 UDG-treated libraries is much less pronounced and 218 present only at the terminal positions of DNA fragments as both C-to-T and G-to-A 219 substitutions (Figure S4.2). For comparison, Figure S4.3 shows DNA damage patterns 220 from previously published shotgun sequences of Spy 94a and Mezmaiskaya 2 individuals. 221 


\section{Denisova 4}
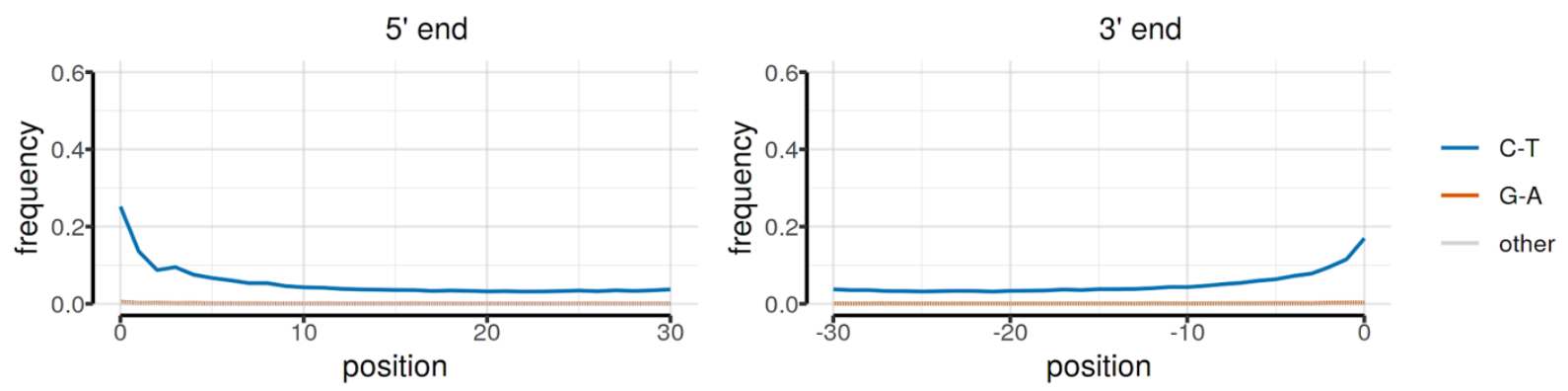

\section{Denisova 8}
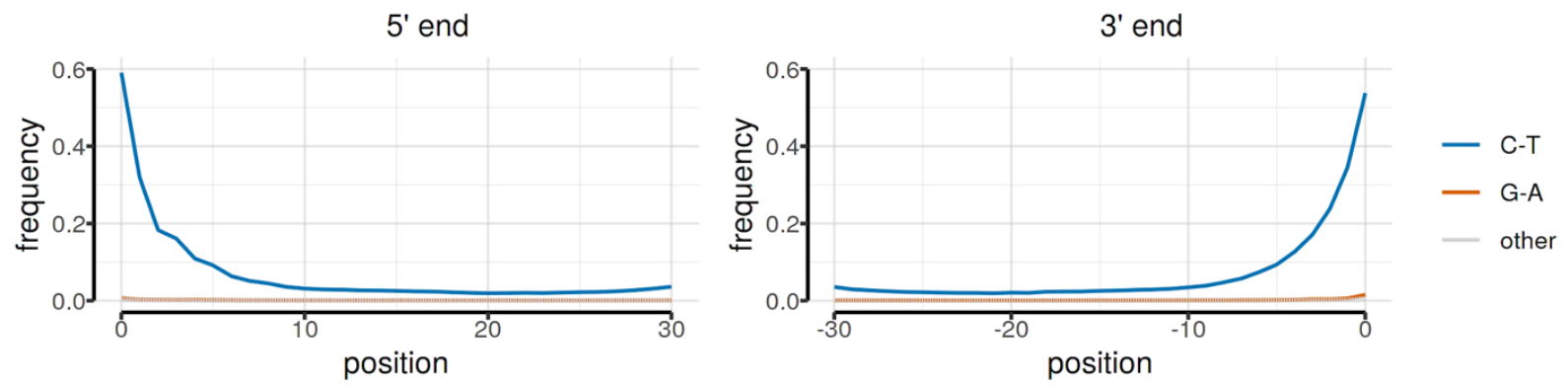

\section{Mezmaiskaya 2}
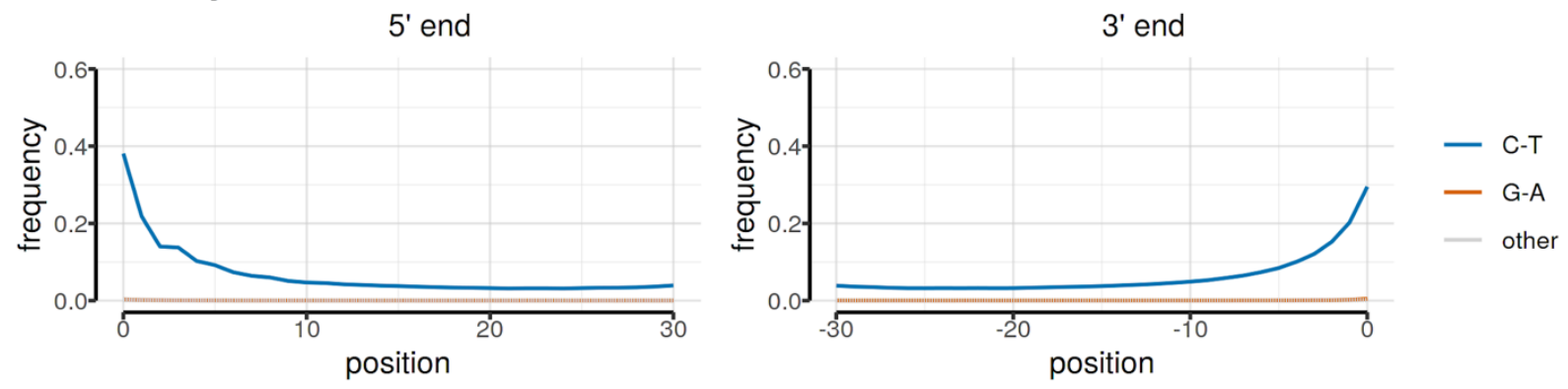

\section{Spy $94 a$}
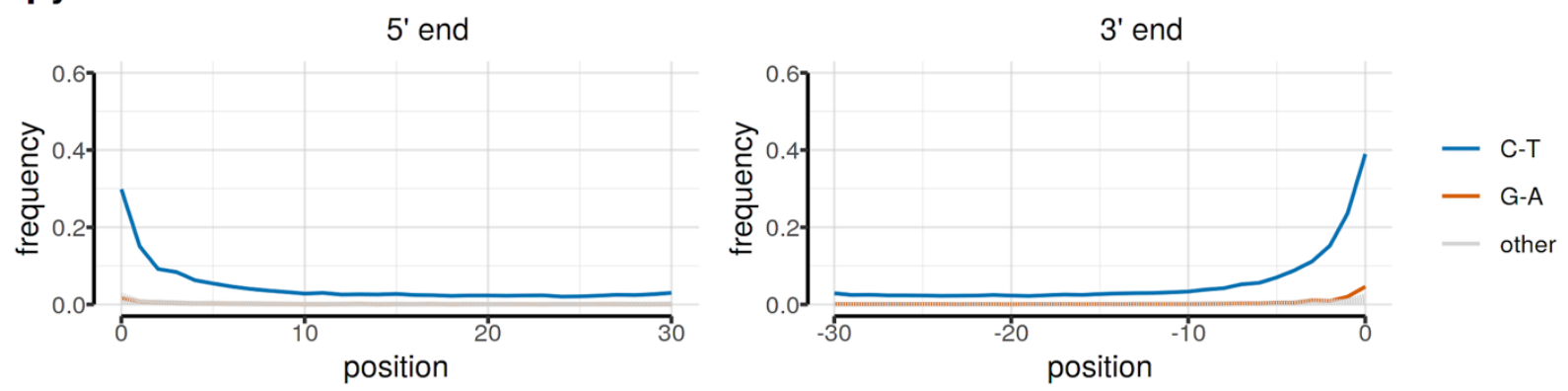

224 Figure S4.1. Patterns of ancient DNA damage in non-UDG-treated sequences 225 captured using the $6.9 \mathrm{Mb}$ capture. 
El Sidrón 1253 (560 kb)

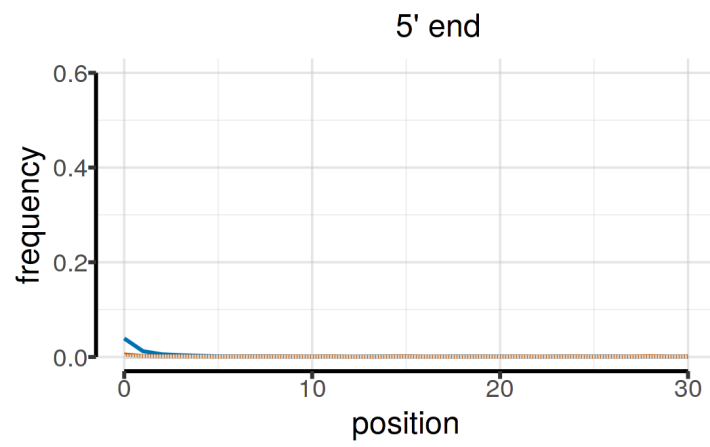

\section{El Sidrón $1253(118$ kb)}

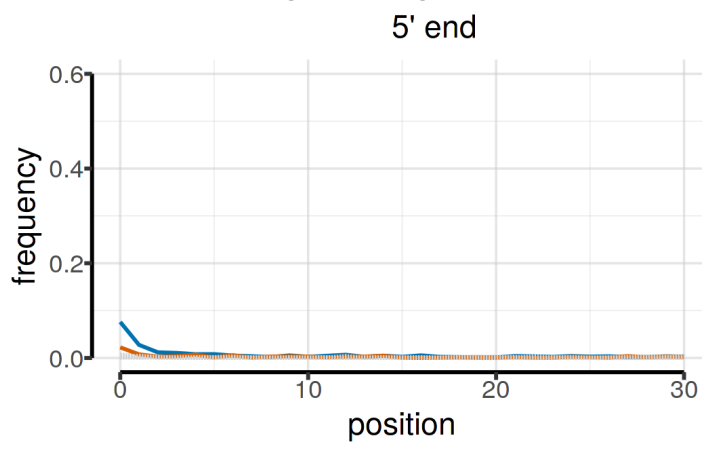

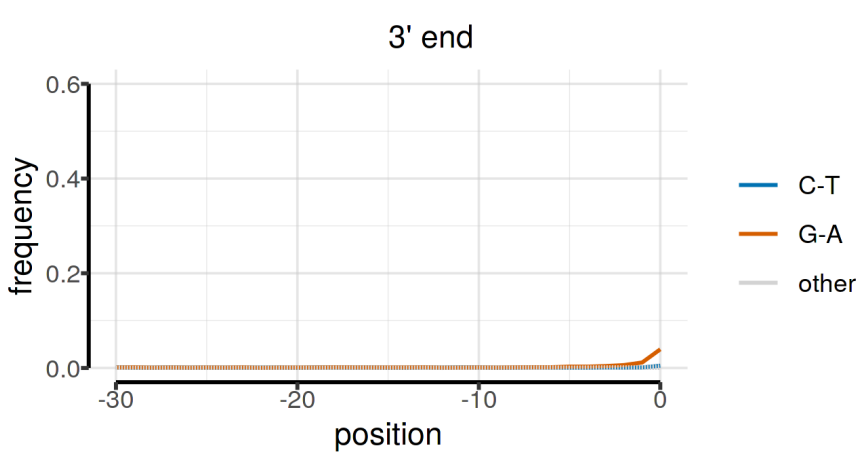

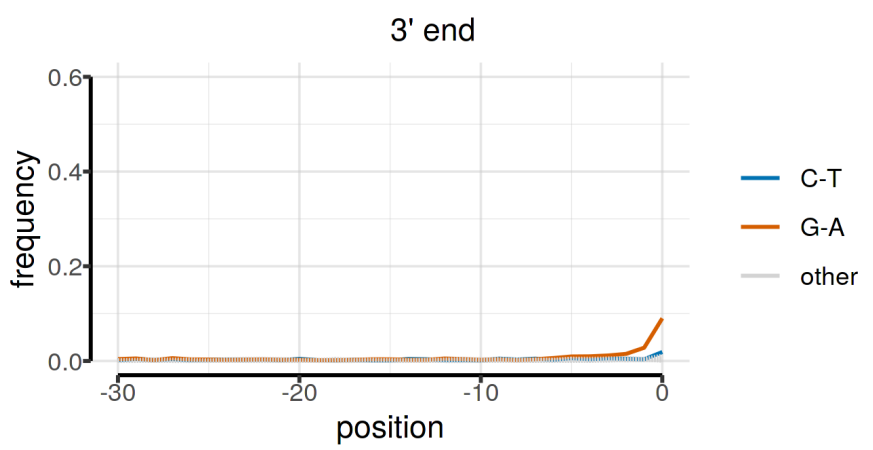

Figure S4.2. Patterns of ancient DNA damage in UDG-treated sequences from the

231 El Sidrón 1253 individual. Top row shows deamination patterns in the 560 kb capture

232 generated for our study (SI 1), bottom row shows deamination patterns in previously 233 published $\mathrm{Y}$ chromosome sequences from the exome capture of the same individual (4, $2345)$. 
Spy 94a (shotgun)

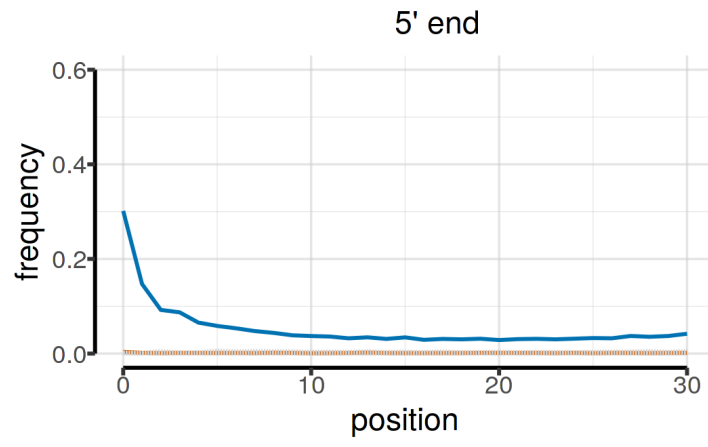

Mezmaiskaya 2 (shotgun)

5 ' end

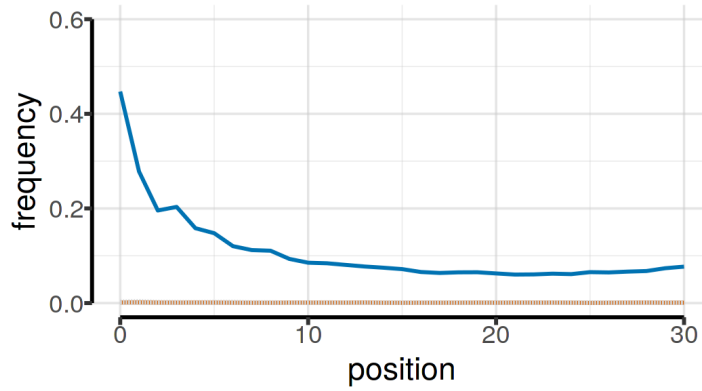

3' end

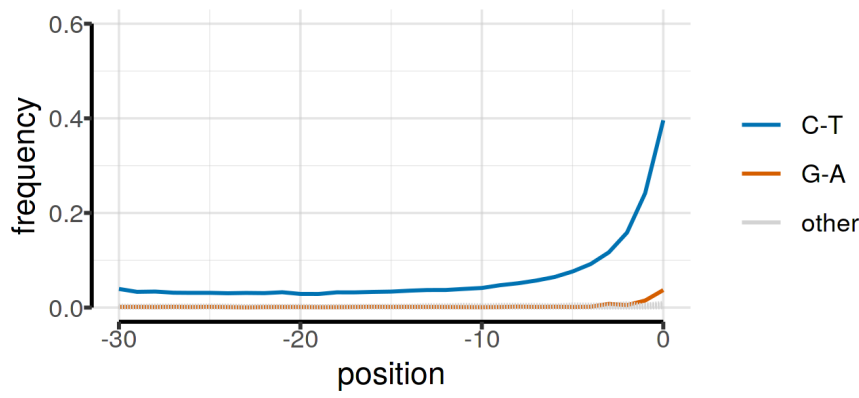

3' end

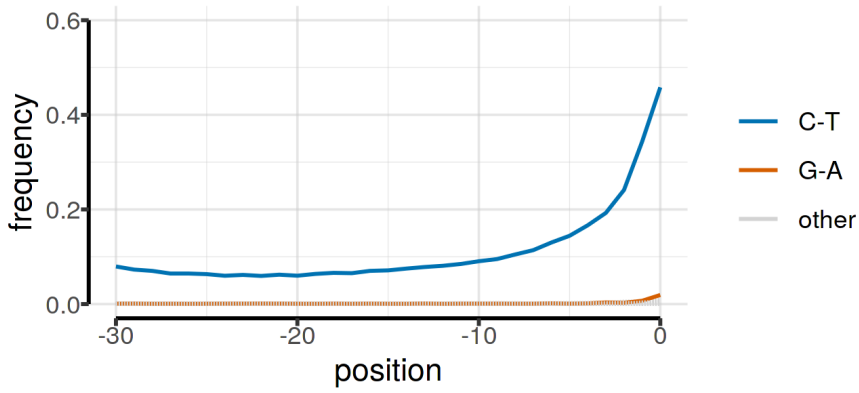

238 Figure S4.3. Patterns of ancient DNA damage in non-UDG-treated shotgun 
246 We calculated read lengths for each final processed BAM file using samtools view and

$247 a w k$. As expected for ancient sequences, archaic human $Y$ chromosome fragments are

248 very short (Figure S4.3, Table S4.4). We note that Denisova 8 shows an even more 249 extreme reduction in read length compared to the other captured archaic human $Y$ 250 chromosomes (Figure S4.3, Table 4.4), consistent with the fact that the Denisova 8 251 specimen is possibly nearly twice as old as the other archaic humans in our study (Figure $2521 \mathrm{~A})$. 


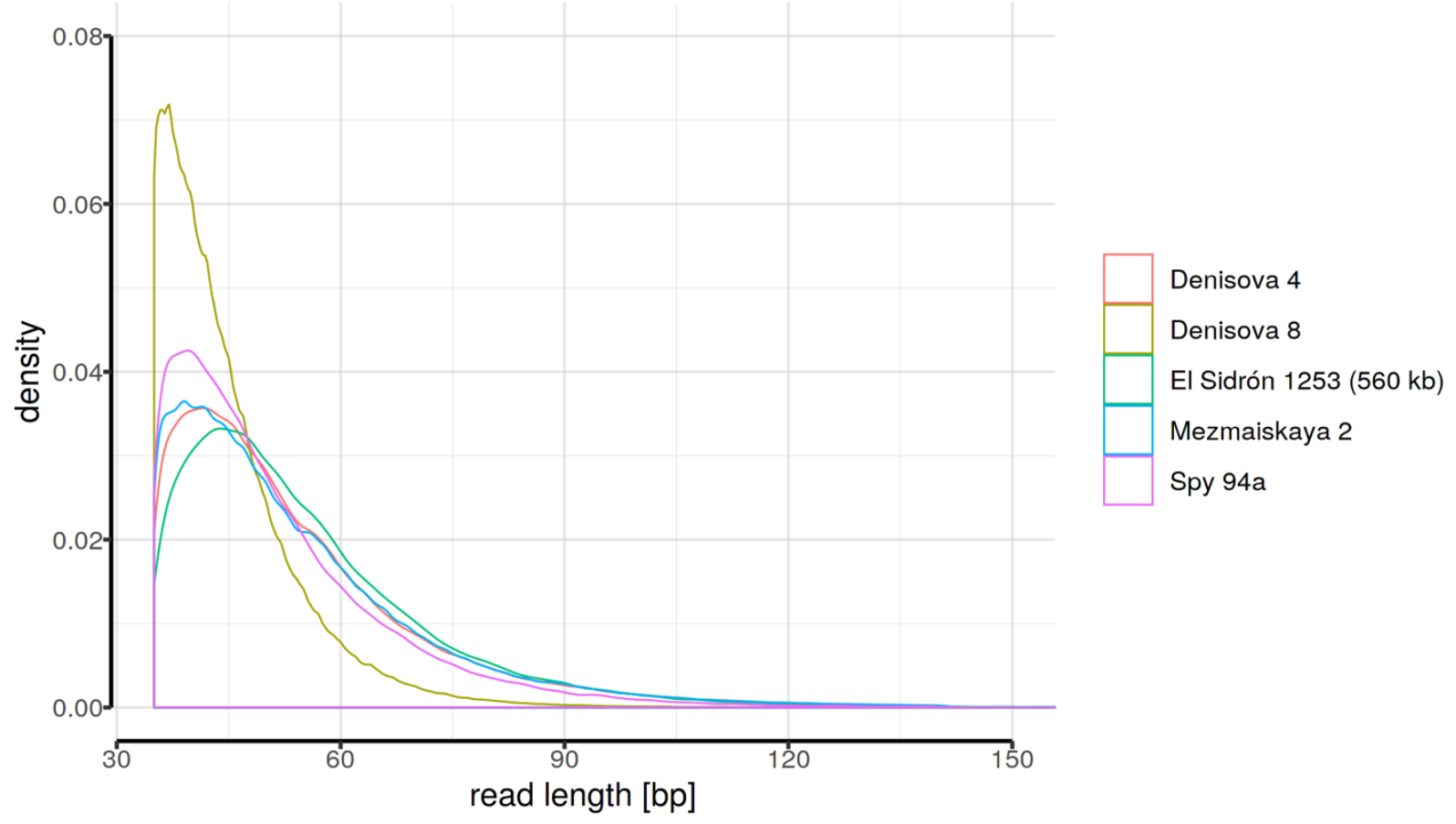




\begin{tabular}{|l|r|r|}
\hline individual & mean [bp] & median [bp] \\
\hline Denisova 8 & 44.8 & 42 \\
\hline Spy 94a & 51.2 & 47 \\
\hline Mezmaiskaya 2 & 54.1 & 49 \\
\hline Denisova 4 & 54.1 & 50 \\
\hline El Sidrón 1253 (560 kb) & 55.3 & 51 \\
\hline
\end{tabular}

262 Table S4.4. Mean and median values of read length distributions in Figure S4.3. 


\subsection{Modern human contamination}

266

267

268

269

270

271

272

273

274

275

276

277

278

279

280

281

282

283

284

285

286

Our consensus-based genotype calling strategy (described in SI 5) is designed to remove the effect of modern human contamination under the assumption that contaminant reads at a given position never represent more than $90 \%$ of the total number of reads. To validate that this approach achieves the desired effect, we assessed the frequency of modern human-derived SNPs at positions informative about modern human contamination in the final archaic human $\mathrm{Y}$ chromosome genotype calls.

To define these informative positions, we used genotypes of present-day human

Y chromosomes and identified ancestral states by determining which sites carry the same allele in chimpanzee and two present-day African lineages A00 and S_Ju_hoan_North-1 $(20,21)$ (red branches in Figure S4.4). We then further restricted these sites to those in which a different allele is observed in all 13 non-African individuals from the SGDP panel (20). These represent alleles derived on the non-African $Y$ chromosome lineage (blue branches in Figure S4.4). This conditioning led to a total of 268 informative positions. Given that all archaic human $Y$ chromosomes are expected to carry the ancestral state at these sites because they all fall basal to modern human $Y$ chromosomes (Figure 2A), observing a derived allele at any of these informative sites implies the presence of a modern human contaminant allele, double mutation or an erroneous SNP call. We note that although the 13 non-African $Y$ chromosomes that we used to define the potential 'contaminant-derived states' may not represent the true contaminant population, the contaminating population would still share the same derived states due to the nonrecombining nature of human $\mathrm{Y}$ chromosomes. 
288 chromosomes carry the ancestral state at all informative positions except for a single

289 position in the Spy 94a individual which shows a derived allele out of the total 16

290 informative sites available (Table S4.5). This shows that the consensus genotype calling

291 method is efficient in mitigating the effect of modern human contaminant reads on the 292 final set of Y chromosome genotype calls. 


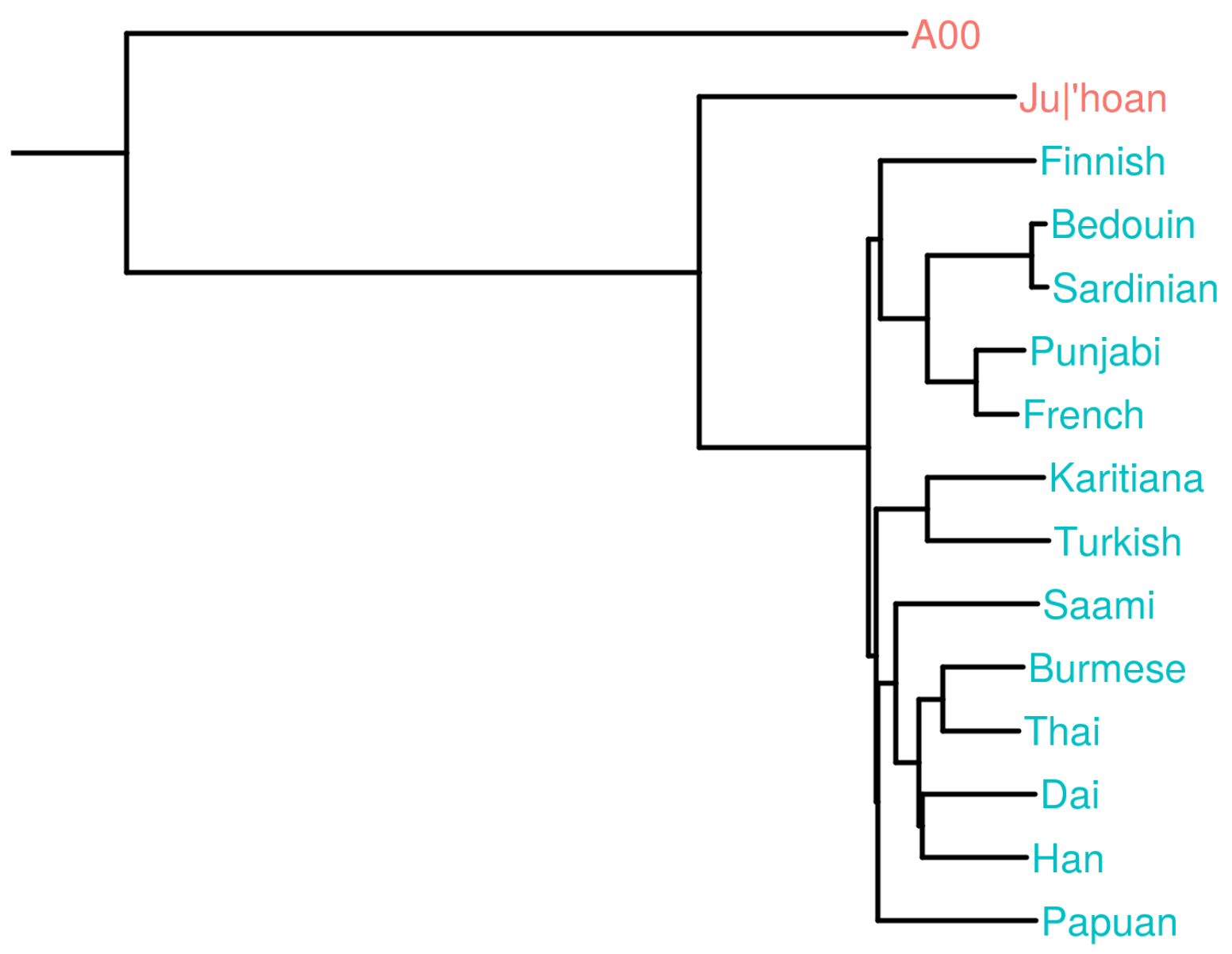

295 Figure S4.4. Phylogenetic tree demonstrating the definition of positions 296 informative about modern human contamination. Red branches represent lineages 297 which we required to carry one state (together with the chimpanzee) at positions where 298 the blue lineages carry a different state. Therefore, red branches represent the ancestral 299 states and blue branches represent the derived state. The tree was rooted with a 300 chimpanzee $\mathrm{Y}$ chromosome as an outgroup (cropped for plotting purposes). 


\begin{tabular}{|l|r|r|r|r|}
\hline \multicolumn{1}{|c|}{ individual } & $\begin{array}{c}\text { ancestral } \\
\text { count }\end{array}$ & $\begin{array}{c}\text { derived } \\
\text { count }\end{array}$ & total & derived/total \\
\hline Spy 94a & 15 & 1 & 16 & 0.0625 \\
\hline Mezmaiskaya 2 & 189 & 0 & 189 & 0.0000 \\
\hline Denisova 4 & 14 & 0 & 14 & 0.0000 \\
\hline Denisova 8 & 90 & 0 & 90 & 0.0000 \\
\hline El Sidrón 1253 (560 kb) & 29 & 0 & 29 & 0.0000 \\
\hline
\end{tabular}

302

303

304 Table S4.5. Counts and proportions of potential 'contaminant-derived' non-African

305 alleles in all archaic human $\mathrm{Y}$ chromosomes.

306 


\subsection{Capture bias and reference (mapping) bias}

Because the probes we designed for $Y$ chromosome DNA enrichment are based on the human reference genome sequence (SI 1), we were concerned about the effect of capture bias on our inferences, specifically on the observed differences in divergence

311 times between Denisovan and Neandertal $Y$ chromosomes with respect to present-day humans (Figure 2). An earlier study of reference bias in published aDNA data sets has found minor but significant allelic imbalances at heterozygous sites from a baseline expectation of $50 \%$ ratio between reference and alternative alleles (26). Because this approach is not applicable for haploid $Y$ chromosomes, we instead looked for departures

316 of the observed number of sites without any genomic coverage from the theoretical 317 expectation.

To build an intuition about this expectation, let's first consider a case of a truly random distribution of sequencing reads in a complete absence of capture or reference bias. In such a situation, the count of reads observed at any site can be modeled as a

321 random variable which follows a Poisson distribution with a parameter $\lambda$, where $\lambda$ 322 represents the average coverage observed across all sites. In a mathematical notation, 323 letting $X$ be this count of reads: $X \sim \operatorname{Poisson}(\lambda)$. Then, given some value of $\lambda$, the 324 expected proportion of sites that are not covered by any sequencing reads can be 325 expressed as Poisson $(X=0, \lambda)$, i.e. as the probability of observing zero reads at any site 326 given the overall average coverage of $\lambda$. As an example, assuming 1 -fold sequencing 327 coverage we would expect to see Poisson $(X=0, \lambda=1)=0.3678794 \sim 37 \%$ of the target 328 sites not to be covered by any read at all, just by random chance. Importantly, however, 329 capture bias or reference bias will manifest by some regions of the genome being 
330 underrepresented in terms of captured molecules or mapped reads. Therefore, the 331 presence and magnitude of this bias in a given DNA enrichment experiment can be 332 detected by estimating the difference between the proportion of sites without any 333 sequencing coverage from the theoretical Poisson expectation.

334 The results, shown in Figure S4.5 and Table S4.6, demonstrate that there is both 335 reference and capture bias in our data and offers several interesting insights. First, we 336 see a comparable effect of bias in all capture data (4-6\% departure from the theoretical 337 Poisson expectation) regardless of which capture array was used for the enrichment 338 procedure (i.e., the full $6.9 \mathrm{Mb}$ capture array, the $560 \mathrm{~kb}$ capture array or the exome 339 capture array, Figure S4.5). Furthermore, comparisons of capture and shotgun 340 sequences of Spy 94a and Mezmaiskaya 2 show that the majority of bias must be due to 341 the capture procedure itself (i.e. failure to capture molecules). This is because the 342 underlying true biological divergences of Spy 94a and Mezmaiskaya 2 to the reference 343 genome (which cause a failure to map reads due to an increased number of substitutions 344 - i.e., a reference bias) must be the same for both capture and shotgun sequences from 345 these individuals. Crucially, however, despite the differences in bias between capture and 346 shotgun sequences, both datasets lead to the same estimates of TMRCA with present347 day human $\mathrm{Y}$ chromosomes (Figure S7.11). Furthermore, although we see dramatically 348 different phylogenetic relationships of Denisovan and Neandertal $Y$ chromosomes with 349 respect to modern humans (Figure 2A), both groups of archaic human capture sequences 350 display comparable magnitudes of both sources of bias (Figure S4.5). Therefore, 351 although undoubtedly present, capture and reference biases cannot result in the 
bioRxiv preprint doi: https://doi.org/10.1101/2020.03.09.983445. this version posted March 9, 2020. The copyright holder for this preprint (which was not certified by peer review) is the author/funder. It is made available under a CC-BY 4.0 International license.

352 observed differences in divergence times between archaic human $Y$ chromosomes and

353 present-day human $\mathrm{Y}$ chromosomes (Figure 2).

354 


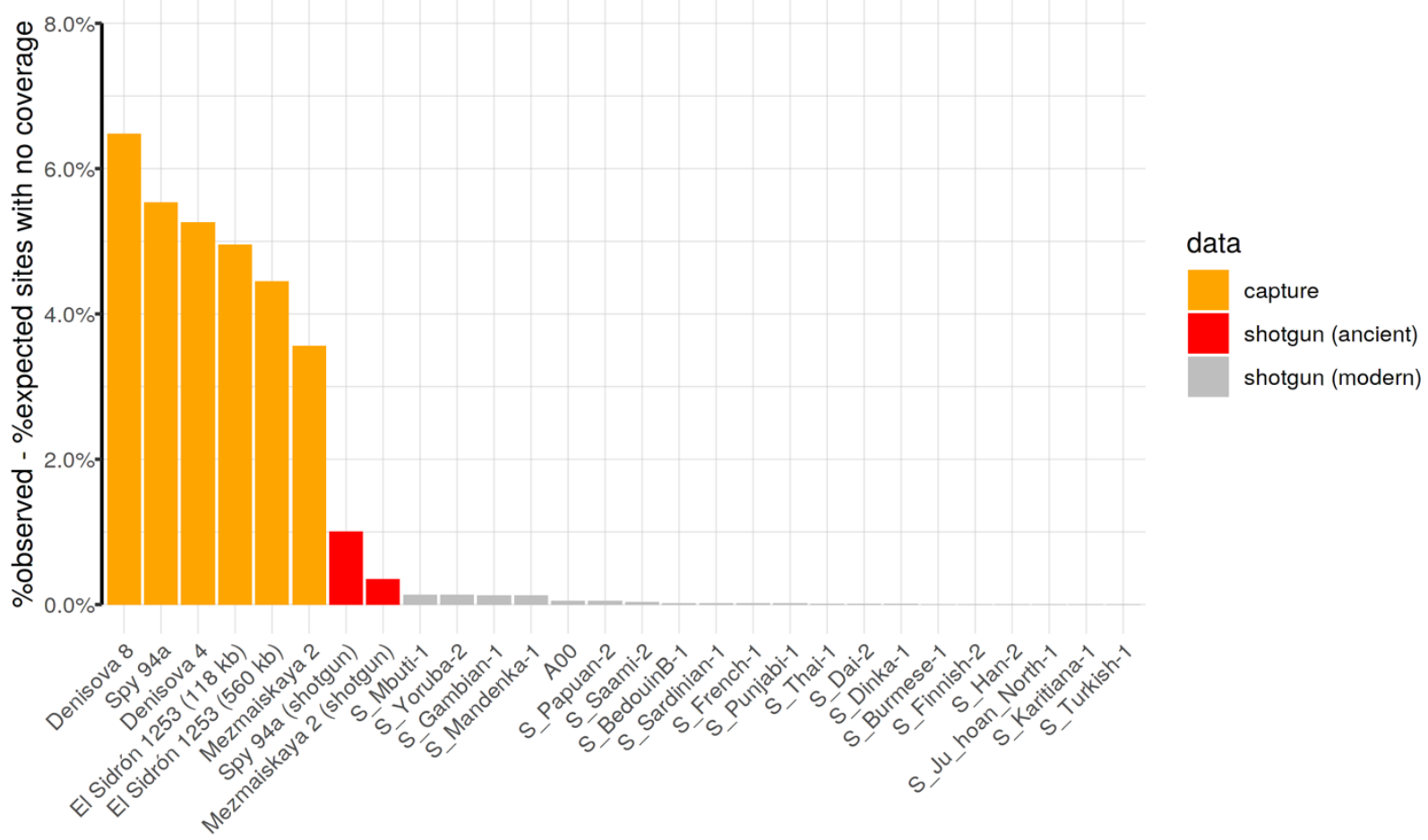

356 Figure S4.5. Differences between expected and observed counts of sites without 357 any sequencing coverage. Exact counts are reported in Table S4.6. Data were filtered 358 according to the criteria described in SI 4.1. 


\begin{tabular}{|c|c|c|c|c|c|}
\hline name & coverage & observed & expected & difference & data \\
\hline Denisova 8 & 3.4820355 & 0.09555 & 0.03074 & 0.06481 & capture \\
\hline Spy $94 a$ & 0.8253622 & 0.49341 & 0.43808 & 0.05533 & capture \\
\hline Denisova 4 & 1.3717806 & 0.30622 & 0.25365 & 0.05257 & capture \\
\hline El Sidrón 1253 (118 kb) & 3.2121215 & 0.08978 & 0.04027 & 0.04951 & capture \\
\hline El Sidrón 1253 (560kb) & 7.9165032 & 0.04479 & 0.00036 & 0.04443 & capture \\
\hline Mezmaiskaya 2 & 14.3494002 & 0.03561 & 0.00000 & 0.03561 & capture \\
\hline Spy $94 a$ (shotgun) & 0.5052873 & 0.61337 & 0.60333 & 0.01004 & shotgun (ancient) \\
\hline Mezmaiskaya 2 (shotgun) & 0.8248822 & 0.44174 & 0.43829 & 0.00345 & shotgun (ancient) \\
\hline S_Yoruba-2 & 16.9616115 & 0.00129 & 0.00000 & 0.00129 & shotgun (modern) \\
\hline S_Mbuti-1 & 20.3360989 & 0.00129 & 0.00000 & 0.00129 & shotgun (modern) \\
\hline S_Mandenka-1 & 16.3312730 & 0.00127 & 0.00000 & 0.00127 & shotgun (modern) \\
\hline S_Gambian-1 & 20.1210272 & 0.00127 & 0.00000 & 0.00127 & shotgun (modern) \\
\hline A00 & 20.8671570 & 0.00050 & 0.00000 & 0.00050 & shotgun (modern) \\
\hline S_Papuan-2 & 23.1529894 & 0.00048 & 0.00000 & 0.00048 & shotgun (modern) \\
\hline S_Saami-2 & 22.6298230 & 0.00033 & 0.00000 & 0.00033 & shotgun (modern) \\
\hline S_BedouinB-1 & 21.7140764 & 0.00016 & 0.00000 & 0.00016 & shotgun (modern) \\
\hline S_Sardinian-1 & 18.1189362 & 0.00015 & 0.00000 & 0.00015 & shotgun (modern) \\
\hline S_Punjabi-1 & 17.1469680 & 0.00013 & 0.00000 & 0.00013 & shotgun (modern) \\
\hline S_French-1 & 21.9031161 & 0.00013 & 0.00000 & 0.00013 & shotgun (modern) \\
\hline S_Thai-1 & 25.1203313 & 0.00010 & 0.00000 & 0.00010 & shotgun (modern) \\
\hline S_Dai-2 & 19.8120096 & 0.00004 & 0.00000 & 0.00004 & shotgun (modern) \\
\hline S_Dinka-1 & 20.7590166 & 0.00004 & 0.00000 & 0.00004 & shotgun (modern) \\
\hline S_Finnish-2 & 17.0155033 & 0.00003 & 0.00000 & 0.00003 & shotgun (modern) \\
\hline S_Han-2 & 20.7680004 & 0.00003 & 0.00000 & 0.00003 & shotgun (modern) \\
\hline S_Karitiana-1 & 22.1963029 & 0.00003 & 0.00000 & 0.00003 & shotgun (modern) \\
\hline S_Turkish-1 & 22.4638799 & 0.00003 & 0.00000 & 0.00003 & shotgun (modern) \\
\hline S_Ju_hoan_North-1 & 22.7025717 & 0.00003 & 0.00000 & 0.00003 & shotgun (modern) \\
\hline S_Burmese-1 & 29.2087547 & 0.00003 & 0.00000 & 0.00003 & shotgun (modern) \\
\hline
\end{tabular}




\section{Genotype calling}

\subsection{Consensus genotype calling}

370

371

372

373

374

375

376

377

378

379

380

381

382

383

384

385

386

387

388

389

390

The haploid nature of the human $Y$ chromosome alleviates many issues inherent to genotype calling of diploid genomes. Most importantly, given that only one allele is expected to be present at each site of a non-recombining portion of the $Y$ chromosome, observing more than one allele at a site must be the result of sequencing errors, DNA damage, contamination, or misalignment of reads. While such issues present a significant problem for calling diploid genotypes, by making it challenging to distinguish true heterozygous calls from erroneously called heterozygous genotypes $(27,28)$, they are less of an issue for haploid genotyping.

To call genotypes of the archaic and modern human $\mathrm{Y}$ chromosomes in our study, we applied a conservative approach to produce a consensus of sequencing reads. For each $Y$ chromosome BAM file, we performed a pileup of reads at each site (disabling base quality recalibration), filtering out reads with mapping quality less than 25 , ignoring bases with base quality less than 20 and removing reads carrying indels at a pileup position. Then, under the assumption that alleles introduced due to DNA damage, sequencing errors, misalignments, or contamination will be in a minority at each site, we called the allele supported by $90 \%$ of the reads in a pileup as the haploid genotype for that site. For further analyses, we additionally restricted to genotype calls supported by at least three reads, as described in section 5.3. This genotype calling procedure has been implemented as part of a functionality of a program which is freely available for download at https://github.com/bodkan/bam-caller. 


\subsection{Genotype calling using snpAD}

392

393

394

395

396

397

398

399

400

401

402

403

404

405

406

407

408

409

410

411

412

413

The consensus genotype calling approach described in the previous section is quite conservative and does not incorporate an explicit model of DNA damage and sequencing errors. To validate the robustness of our consensus-based results, we compared them to genotype calls generated using snpAD, an aDNA-specific genotype caller (28). A major caveat of this approach is the fact that $s n p A D$ has been designed for calling diploid genotypes and accurate results requires at least $4 \mathrm{X}$ genomic coverage (28). Therefore, its genotype calling model has not been tested on low coverage, haploid chromosomes such as those generated in our study. While recognizing these limitations, we used snpAD to call genotypes of all four archaic $\mathrm{Y}$ chromosomes captured for the $6.9 \mathrm{Mb}$ target regions (Denisova 4, Denisova 8, Spy 94a and Mezmaiskaya 2), discarded any sites which were called as heterozygous (likely the result of errors, contamination or aDNA damage), and converted all homozygous genotype calls to a haploid state.

In accordance with snpAD's more sophisticated model of aDNA damage patterns, we have found that the number of successfully genotyped sites is higher than those generated by our simpler consensus-based genotype calling approach, but only marginally so (Table S5.1). Furthermore, although the rates of C-to-T and G-to-A SNP frequencies observed in the final set of genotype calls of the high coverage Mezmaiskaya 2 is very close to the baseline expectation for present-day DNA, the remaining low coverage archaic $\mathrm{Y}$ chromosomes are still affected by aDNA damage and show an excess of falsely called genotypes (Figures S5.1 and S5.2). We note that this is not unexpected, because the coverage of these individuals is much lower than what is recommended by for snpAD (28). Overall, we did not observe significant differences 
414 between snpAD-based and consensus-based genotypes in terms of the inferred times to 415 the most recent common ancestor (TMRCA) and, in fact, we found that both lead to the 416 same conclusions (Figure S7.11). Based on these analyses we concluded that our

417 conservative $90 \%$ cutoff for consensus genotype calling method is appropriate and 418 decided to use it for all analyses. 


\subsection{Minimum coverage filtering}

421 The majority of libraries analyzed in our study have not been treated with the uracil-

422 deglycosylase (UDG) enzyme (SI 2). Unlike UDG-treated libraries, non-UDG libraries

423 retain an increased deamination signal throughout the molecules (Figures S4.1 and S4.2)

424 which poses a significant challenge for distinguishing false substitutions caused by aDNA 425 damage from true polymorphisms (28).

426 For a given sequencing read carrying a putative substitution, it is not 427 straightforward to decide whether this substitution represents a true polymorphism or 428 error. Given enough sequencing coverage, this issue can be mostly overcome by 429 observing a sufficient number of bases from reads that do not carry a deamination430 induced substitution, integrating evidence from multiple reads at a site (28). However, as 431 our data is of relatively low coverage (Figure 1B, Table S4.1), we were concerned by 432 selecting an appropriate lower coverage cutoff to minimize the impact of false 433 polymorphisms on our inferences. Specifically, if the same nucleotide is observed in a 434 majority of reads mapped to the same genomic position, it is unlikely that this would be 435 the result of aDNA damage, sequencing errors or contamination, as these occur mostly 436 at relatively low frequencies in the individuals in our study (Figure S4.1 and Table S4.5).

437 To get a sense of the frequency of calling false polymorphisms as a function of coverage, 438 we calculated the proportions of observed genotypes in each archaic human $Y$ 439 chromosome given a certain coverage filtering cutoff and compared those to the baseline 440 expectation for present-day human DNA. As expected, allowing SNPs supported by only 441 one read leads to a significant excess of C-to-T and G-to-A SNP (Figure S5.1), a 442 consequence of the presence of aDNA damage (Figure S4.1). We found that increasing 
443 the minimum coverage cutoff to two reads causes the rate of aDNA-induced SNPs to drop

444 significantly towards the baseline expectation, but going beyond requiring the support of

445 three reads for each SNP does not lead to further improvement in accuracy of genotype

446 calling (Figure S5.1). Because of this and because each additional increase in required

447 minimum coverage is at the expense of the final number of available sites, we settled on

448 a minimum coverage cutoff of 3 reads.

449 It is important to note that despite the residual presence of false aDNA 450 substitutions in the final set of filtered genotype calls, manifesting as increased 451 frequencies of C-to-T and G-to-A SNPs compared to present-day DNA (Figure S5.1), 452 comparisons of archaic-modern human TMRCA estimates obtained using the full set of 453 genotype calls and those based on genotypes restricted to non-C-to-T/G-to-A SNPs did 454 not reveal any significant differences (Figure S7.9). This is partially due to very low rates 455 of residual false SNPs that pass through the filtering, but mostly because our TMRCA 456 estimators are quite insensitive to private mutations on the archaic lineage (SI 7). A 457 second validation of our coverage filter follows from the fact that the two Denisovan and 458 all three Neandertal $Y$ chromosomes lead to the same TMRCA estimates with modern 459 human $\mathrm{Y}$ chromosomes despite differences in coverage and rates of aDNA damage 460 (Figures 1B, 2B and S5.1). Both of these factors would affect genotyping accuracy if not 461 handled appropriately, and would introduce noise in TMRCA estimates estimated for 462 individual Y chromosomes.

463 Complete counts of $Y$ chromosome positions passing the filters for all individuals 464 are reported in Tables 5.1 and 5.2 (counts for archaic and modern human individuals, 
bioRxiv preprint doi: https://doi.org/10.1101/2020.03.09.983445. this version posted March 9, 2020. The copyright holder for this preprint (which was not certified by peer review) is the author/funder. It is made available under a CC-BY 4.0 International license.

465 respectively, in $6.9 \mathrm{Mb}$ target regions) and Tables 5.3 and 5.4 (counts for archaic and 466 modern human individuals, respectively, in $560 \mathrm{~kb}$ target regions). 
Denisova 4

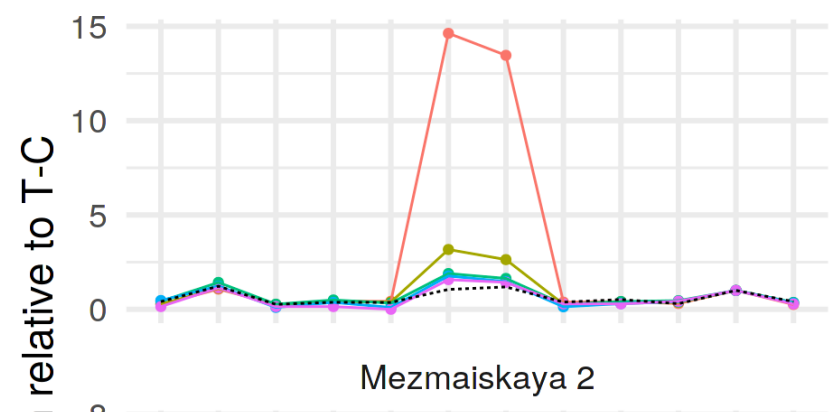

Denisova 8

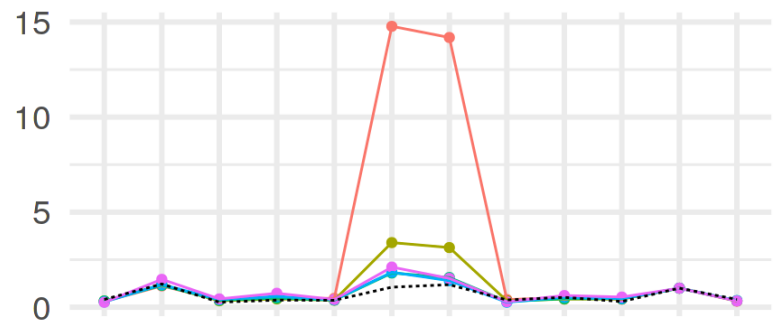

Spy $94 a$

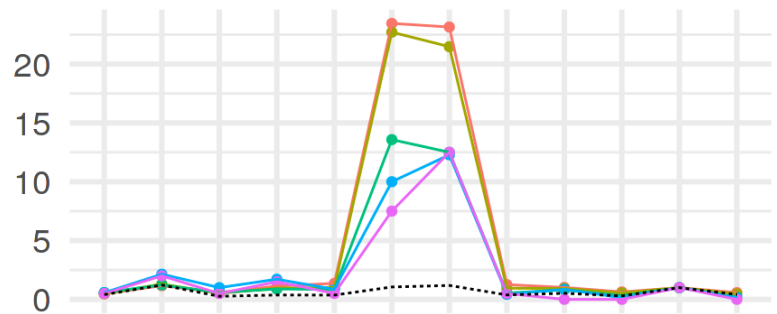

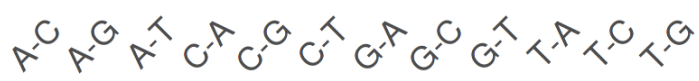

SNP class

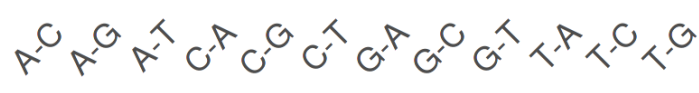

minimum coverage cutoff $\rightarrow 1 \rightarrow 2 \rightarrow 3 \rightarrow 4 \rightarrow 5$

468 Figure S5.1. Frequencies of observed polymorphisms normalized by the observed

469 frequency of T-C polymorphism. SNP classes were counted for each archaic human $Y$

470 chromosome (one panel each) and all counts were normalized by dividing them with

471 observed counts of T-C SNPs. Dotted line shows an expectation based on SNP

472 proportions observed in Y chromosomes of the following SGDP individuals: S_French_1,

473 S_Papuan_2, S_Burmese_1, S_Thai_1, S_Sardinian_1.

474 
Denisova 4 (snpAD)

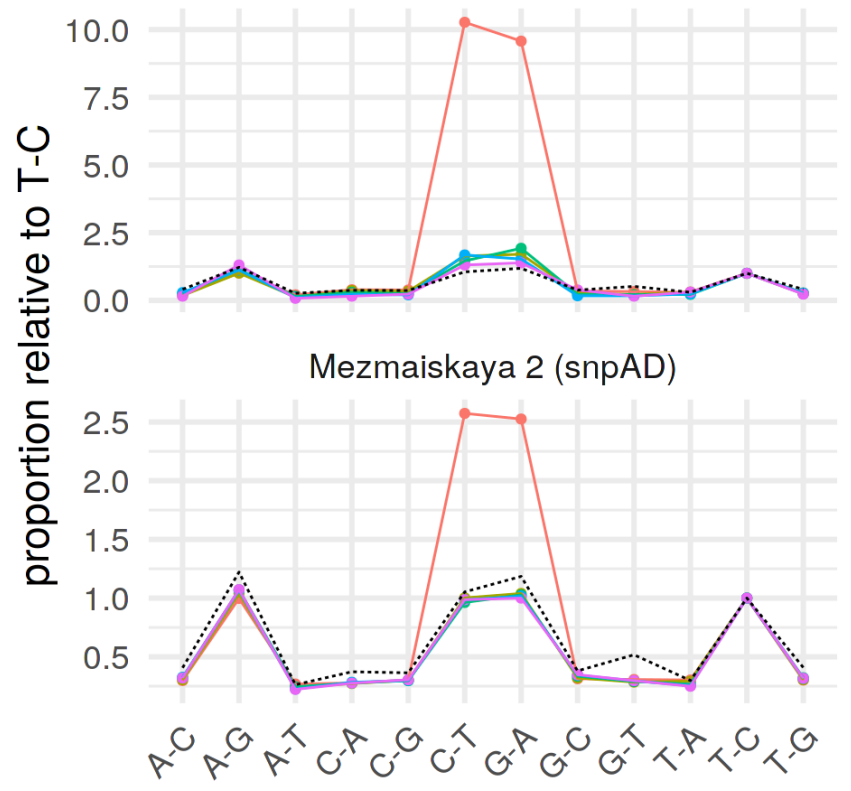

Denisova 8 (snpAD)

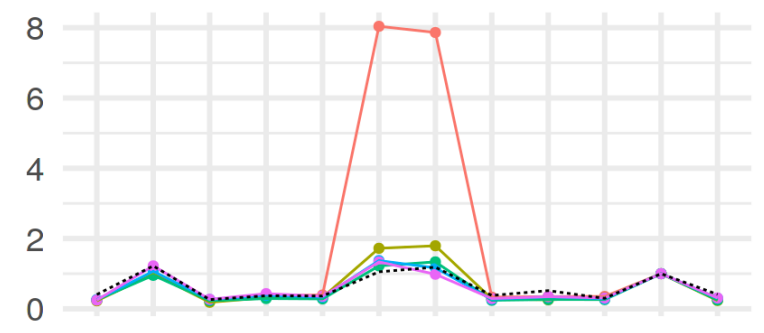

Spy94a (snpAD)

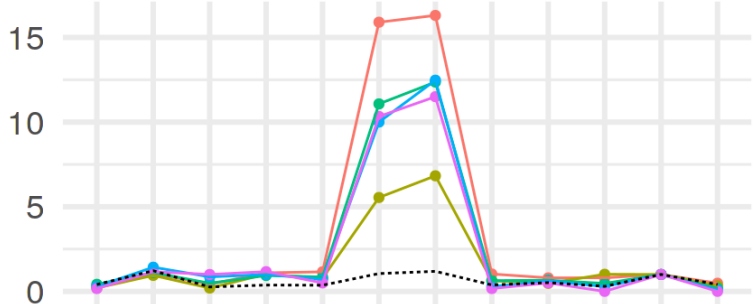

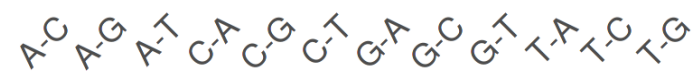

SNP class

minimum coverage cutoff $\rightarrow 1 \rightarrow 2 \rightarrow 3 \rightarrow 4 \rightarrow 5$

476 Figure S5.2. Frequencies of observed polymorphisms normalized by the observed

477 frequency of T-C polymorphism. SNP classes were counted for each archaic human $Y$

478 chromosome (one panel each) and all counts were normalized by dividing them with

479 observed counts of T-C SNPs. Dotted line shows an expectation based on SNP

480 proportions observed in Y chromosomes of the following SGDP individuals: S_French_1, 


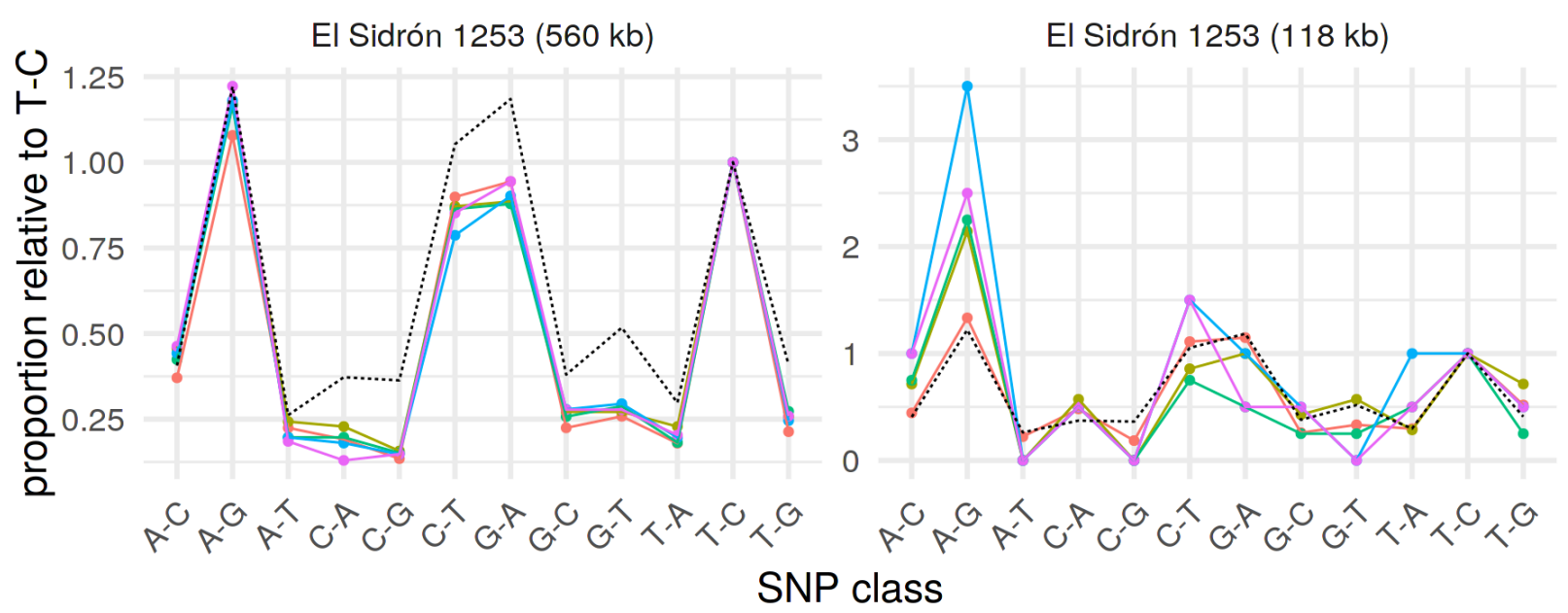

484

minimum coverage cutoff $\rightarrow 1 \rightarrow 2 \rightarrow 3 \rightarrow 4 \rightarrow 5$

Figure S5.3. Frequencies of observed polymorphisms normalized by the frequency of T-C polymorphism. SNP classes were counted for each archaic human $\mathrm{Y}$ 487 chromosome (one panel each) and all counts were normalized by dividing them with 488 observed counts of T-C SNPs. Dotted line shows an expectation based on SNP proportions observed in Y chromosomes of the following SGDP individuals: S_French_1, 


\begin{tabular}{|r|r|r|r|r|}
\hline \multirow{2}{*}{ name } & \multicolumn{2}{|c|}{ all sites } & \multicolumn{2}{c|}{ excluding C-to-T/G-to-A } \\
\cline { 2 - 5 } & \multicolumn{1}{|c|}{ count } & proportion & \multicolumn{1}{c|}{ count } & \multicolumn{1}{c|}{ proportion } \\
\hline Spy 94a (shotgun) & $2,664,787$ & $38.5 \%$ & $2,636,405$ & $38.1 \%$ \\
\hline Spy 94a & $3,502,380$ & $50.7 \%$ & $3,470,671$ & $50.2 \%$ \\
\hline Spy 94a (snpAD) & $3,557,124$ & $51.5 \%$ & $3,522,759$ & $51.0 \%$ \\
\hline Denisova 4 & $4,731,302$ & $68.4 \%$ & $4,705,860$ & $68.1 \%$ \\
\hline Denisova 4 (snpAD) & $4,824,242$ & $69.8 \%$ & $4,798,037$ & $69.4 \%$ \\
\hline Denisova 8 & $5,851,332$ & $84.6 \%$ & $5,828,356$ & $84.3 \%$ \\
\hline Denisova 8 (snpAD) & $6,260,242$ & $90.6 \%$ & $6,236,272$ & $90.2 \%$ \\
\hline Mezmaiskaya 2 (shotgun) & $3,822,106$ & $55.3 \%$ & $3,762,768$ & $54.4 \%$ \\
\hline Mezmaiskaya 2 & $6,348,948$ & $91.8 \%$ & $6,346,684$ & $91.8 \%$ \\
\hline Mezmaiskaya 2 (snpAD) & $6,669,912$ & $96.5 \%$ & $6,667,441$ & $96.5 \%$ \\
\hline
\end{tabular}

498 Table S5.1. Counts of sites for each archaic human $\mathrm{Y}$ chromosome in $6.9 \mathrm{Mb}$ 499 capture regions which passed the filtering for minimum depth of 3 reads in addition to other filtering and genotype calling criteria. Multiple records for the same individual

501 indicate different versions of the data (shotgun sequences as opposed to capture) or 502 different ways of calling genotypes (consensus genotype calling or genotype calling using 503 snpAD). Reported are numbers for all sites and for sites excluding C-T and G-A 504 polymorphisms. The proportions are calculated relative to the total number of available 505 sites $(6,912,728 ;$ SI 1$)$. 


\begin{tabular}{|c|c|c|c|c|}
\hline \multirow{2}{*}{ name } & \multicolumn{2}{|c|}{ all sites } & \multicolumn{2}{|c|}{ excluding C-to-T/G-to-A } \\
\hline & count & proportion & count & proportion \\
\hline$A 00-1$ & $6,852,077$ & $99.12 \%$ & $6,851,175$ & $99.11 \%$ \\
\hline A00-2 & $6,867,616$ & $99.35 \%$ & $6,866,716$ & $99.33 \%$ \\
\hline $\mathrm{A} 00$ & $6,873,078$ & $99.43 \%$ & $6,872,184$ & $99.41 \%$ \\
\hline Ust'-Ishim & $6,888,071$ & $99.64 \%$ & $6,887,986$ & $99.64 \%$ \\
\hline S_Yoruba-2 & $6,897,101$ & $99.77 \%$ & $6,896,820$ & $99.77 \%$ \\
\hline S_Mandenka-1 & $6,898,162$ & $99.79 \%$ & $6,897,879$ & $99.79 \%$ \\
\hline S_Mbuti-1 & $6,898,694$ & $99.80 \%$ & $6,898,400$ & $99.79 \%$ \\
\hline S_Gambian-1 & $6,899,210$ & $99.80 \%$ & $6,898,929$ & $99.80 \%$ \\
\hline S_Papuan-2 & $6,904,393$ & $99.88 \%$ & $6,904,208$ & $99.88 \%$ \\
\hline S_Thai-1 & $6,905,023$ & $99.89 \%$ & $6,904,846$ & $99.89 \%$ \\
\hline S_BedouinB-1 & $6,905,031$ & $99.89 \%$ & $6,904,820$ & $99.89 \%$ \\
\hline S_Saami-2 & $6,905,291$ & $99.89 \%$ & $6,905,102$ & $99.89 \%$ \\
\hline S_Sardinian-1 & $6,905,558$ & $99.90 \%$ & $6,905,352$ & $99.89 \%$ \\
\hline S_Ju_hoan_North-1 & $6,906,228$ & $99.91 \%$ & $6,905,872$ & $99.90 \%$ \\
\hline S_Karitiana-1 & $6,906,409$ & $99.91 \%$ & $6,906,258$ & $99.91 \%$ \\
\hline S_Dai-2 & $6,906,414$ & $99.91 \%$ & $6,906,248$ & $99.91 \%$ \\
\hline S_Burmese-1 & $6,906,438$ & $99.91 \%$ & $6,906,260$ & $99.91 \%$ \\
\hline S_Dinka-1 & $6,906,507$ & $99.91 \%$ & $6,906,225$ & $99.91 \%$ \\
\hline S_French-1 & $6,906,914$ & $99.92 \%$ & $6,906,738$ & $99.91 \%$ \\
\hline S_Punjabi-1 & $6,907,065$ & $99.92 \%$ & $6,906,885$ & $99.92 \%$ \\
\hline S_Finnish-2 & $6,907,088$ & $99.92 \%$ & $6,906,889$ & $99.92 \%$ \\
\hline S_Han-2 & $6,907,603$ & $99.93 \%$ & $6,907,417$ & $99.92 \%$ \\
\hline S_Turkish-1 & $6,907,751$ & $99.93 \%$ & $6,907,717$ & $99.93 \%$ \\
\hline
\end{tabular}


509 Table S5.2. Counts of sites for each modern human $Y$ chromosome (consensus

510 genotype calls of shotgun data) in $6.9 \mathrm{Mb}$ capture regions which passed the

511 filtering for minimum depth of 3 reads in addition to other filtering and genotype

512 calling criteria. Reported are numbers for all sites and for sites excluding C-T and G-A

513 polymorphisms. The proportions are calculated relative to the total number of available 514 sites $(6,912,728)$. 


\begin{tabular}{|r|r|r|r|r|}
\hline \multirow{2}{*}{ name } & \multicolumn{2}{|c|}{ all sites } & excluding C-to-T/G-to-A \\
\cline { 2 - 5 } & count & proportion & count & proportion \\
\hline Spy 94a & 275,626 & $49.55 \%$ & 272,798 & $49.04 \%$ \\
\hline Denisova 4 & 408,775 & $73.49 \%$ & 406,508 & $73.08 \%$ \\
\hline Denisova 8 & 484,377 & $87.08 \%$ & 482,608 & $86.76 \%$ \\
\hline Mezmaiskaya 2 & 509,145 & $91.53 \%$ & 508,962 & $91.50 \%$ \\
\hline El Sidrón 1253 (560 kb) & 530,172 & $95.31 \%$ & 530,046 & $95.29 \%$ \\
\hline
\end{tabular}

518 Table S5.3. Counts of sites for each archaic human Y chromosome (in 560 kb 519 capture regions) which passed the filtering for minimum depth of 3 reads in 520 addition to other filtering and genotype calling criteria. Reported are numbers for all

521 sites and for sites excluding C-to-T and G-to-A polymorphisms. The proportions are 522 calculated relative to the total number of available sites $(556,259)$. 


\begin{tabular}{|c|c|c|c|c|}
\hline \multirow{2}{*}{ name } & \multicolumn{2}{|c|}{ all sites } & \multicolumn{2}{|c|}{ excluding C-to-T and G-to-A } \\
\hline & count & proportion & count & proportion \\
\hline $\mathrm{A} 00-1$ & 552,557 & $99.33 \%$ & 552,456 & $99.32 \%$ \\
\hline S_Yoruba-2 & 553,203 & $99.45 \%$ & 553,112 & $99.43 \%$ \\
\hline S_Mandenka-1 & 553,348 & $99.48 \%$ & 553,257 & $99.46 \%$ \\
\hline S_Gambian-1 & 553,365 & $99.48 \%$ & 553,275 & $99.46 \%$ \\
\hline S_Mbuti-1 & 553,381 & $99.48 \%$ & 553,288 & $99.47 \%$ \\
\hline $\mathrm{A} 00-2$ & 553,422 & $99.49 \%$ & 553,321 & $99.47 \%$ \\
\hline $\mathrm{A} 00$ & 553,618 & $99.53 \%$ & 553,517 & $99.51 \%$ \\
\hline Ust'-Ishim & 554,575 & $99.70 \%$ & 554,550 & $99.69 \%$ \\
\hline S_Thai-1 & 555,830 & $99.92 \%$ & 555,787 & $99.92 \%$ \\
\hline S_Dinka-1 & 555,842 & $99.93 \%$ & 555,785 & $99.91 \%$ \\
\hline S_BedouinB-1 & 555,850 & $99.93 \%$ & 555,806 & $99.92 \%$ \\
\hline S_Sardinian-1 & 555,855 & $99.93 \%$ & 555,813 & $99.92 \%$ \\
\hline S_Burmese-1 & 555,862 & $99.93 \%$ & 555,818 & $99.92 \%$ \\
\hline S_Dai-2 & 555,864 & $99.93 \%$ & 555,819 & $99.92 \%$ \\
\hline S_Ju_hoan_North-1 & 555,870 & $99.93 \%$ & 555,818 & $99.92 \%$ \\
\hline S_Karitiana-1 & 555,876 & $99.93 \%$ & 555,848 & $99.93 \%$ \\
\hline S_Saami-2 & 555,927 & $99.94 \%$ & 555,891 & $99.93 \%$ \\
\hline S_French-1 & 555,942 & $99.94 \%$ & 555,902 & $99.94 \%$ \\
\hline S_Finnish-2 & 555,945 & $99.94 \%$ & 555,906 & $99.94 \%$ \\
\hline S_Papuan-2 & 555,951 & $99.94 \%$ & 555,918 & $99.94 \%$ \\
\hline S_Punjabi-1 & 555,958 & $99.95 \%$ & 555,920 & $99.94 \%$ \\
\hline S_Turkish-1 & 555,971 & $99.95 \%$ & 555,966 & $99.95 \%$ \\
\hline S_Han-2 & 556,022 & $99.96 \%$ & 555,978 & $99.95 \%$ \\
\hline
\end{tabular}

525 Table S5.4. Counts of sites for each modern human Y chromosome (in 560 kb

526 capture regions) which passed the filtering for minimum depth of 3 reads in

527 addition to other filtering and genotype calling criteria. Reported are numbers for all 
bioRxiv preprint doi: https://doi.org/10.1101/2020.03.09.983445. this version posted March 9, 2020. The copyright holder for this preprint (which was not certified by peer review) is the author/funder. It is made available under a CC-BY 4.0 International license.

528 sites and for sites excluding C-to-T and G-to-A polymorphisms. The proportions are

529 calculated relative to the total number of available sites $(556,259)$. 


\section{$531 \quad 6 . \quad$ Inferring phylogenetic relationships}

532 To resolve the phylogenetic relationships of each archaic human $Y$ chromosome to all

533 other $\mathrm{Y}$ chromosome sequences, we merged the VCF files with genotype calls from each

534 individual (including the chimpanzee) into a single VCF file and converted the genotypes

535 to the FASTA format using a custom Python script (available on our Github repository:

536 https://github.com/bodkan/archaic-ychr). To mitigate biases introduced by low coverage

537 and characteristics of aDNA damage (29), we excluded all C-to-T and G-to-A 538 polymorphisms and applied the same filters for each individual as for all other analyses 539 in our study. Finally, we excluded monomorphic sites and sites carrying private changes 540 on the chimpanzee lineage to reduce the size of the final alignment file.

541 To construct a neighbor-joining phylogenetic tree (Figure 2A), we utilized the

542 functionality provided by R packages ape and phangorn (30, 31). First, we calculated the

543 distance matrix between all $Y$ chromosome pairs in the FASTA file with the function

544 dist.dna, using the model of simple pairwise differences (model = "raw") and

545 excluding sites with missing data specific to each pair (pairwise. deletion = TRUE).

546 We then provided this distance matrix to the $n j$ function and rooted the resulting

547 neighbor-joining tree using the function midpoint from the phangorn package. Bootstrap

548 confidence numbers for the neighbor-joining tree were calculated using ape's

549 boot.phylo function over 100 replicates. After inspecting the resulting phylogenetic

550 tree, we found that the private branch leading to the Denisova 4 had a negative length

551 (value $=-0.00088$ ). Given that negative branch lengths are a relatively common artefact

552 of the neighbor-joining algorithm and do not affect the reliability of the generated tree we

553 followed the recommendation to set the branch length to zero (32). We note that this does 
554 not have any impact on our conclusions, because the change involves a private branch

555 whose length is not meaningful given the discrepancies between sample dates and

556 implied tree tip dates (Figures $1 \mathrm{~A}$ and $2 \mathrm{~A},(29)$ ). The final trees were annotated and

557 plotted using the R package ggtree (33).

558

559 


\section{Estimating the TMRCA of archaic and modern human $Y$}

\section{chromosomes}

562 Given that most of the $\mathrm{Y}$ chromosome capture data analyzed in our study is of relatively

563 low coverage (Figure 1B, Table S4.1), care needs to be taken when estimating

564 phylogenetic parameters such as the time to the most recent common ancestor (TMRCA).

565 Similarly, low coverage and the associated reduction in the accuracy of genotype calls

566 render the inferred aDNA branch lengths unreliable (29). Any phylogenetic method of

567 choice must be therefore robust to sequencing errors and incorrect branch lengths. We

568 also observe discordances between sample dates and implied molecular tip dates, likely

569 due to residual genotype calling errors (compare Figure 1A vs Figure $2 \mathrm{~A}$ ). We therefore

570 estimated TMRCAs between archaic and modern human $\mathrm{Y}$ chromosomes using a

571 method inspired by the analysis of the El Sidrón 1253 Neandertal coding sequence (5).

572 Instead of using polymorphisms on the archaic human lineage, this method relies on first

573 estimating the TMRCA of a pair of high-coverage African and non-African $Y$

574 chromosomes $\left(T M R C A_{A F R}\right)$ which is then used to extrapolate the deeper divergence time

575 between archaic and modern human $Y$ chromosomes. We describe the method in the

576 sections below, detailing our modifications and improvements. 


\subsection{TMRCA of Africans and non-Africans $T M R C A_{A F R}$}

580 The original study by Mendez et al. estimated the TMRCA between the A00 African $Y$ 581 chromosome lineage and the hg19 $\mathrm{Y}$ chromosome as a representative of non-African $\mathrm{Y}$ 582 chromosomes $(5,21)$. In order to get a better sense of the uncertainty and noise in our

583 TMRCA estimates, we expanded the present-day $Y$ chromosome reference panel to 13 584 non-African and 6 African $Y$ chromosomes from the SGDP data set (Table S7.1) (20). In the first step, we estimated mutation rate in the $6.9 \mathrm{Mb}$ capture target using the high-coverage $Y$ chromosome of Ust'-Ishim, a 45,000 years old hunter-gatherer from

587 Siberia (22). We counted derived mutations missing on the Ust'-Ishim branch compared 588 to those observed in the panel of 13 non-African $Y$ chromosomes, and used this branch589 shortening to calculate mutation rate assuming generation time of 25 years (Figure S7.1, 590 Table S7.2). In the second step, we counted mutations accumulated on an African lineage 591 and a non-African lineage since their split from each other and calculated the TMRCA of 592 both (in units of years ago) using the mutation rate estimated in the first step. Importantly, 593 we discovered that the branch-lengths in Africans are as much as $13 \%$ shorter compared 594 to non-Africans (Figure S7.3), which is consistent with significant branch length variability 595 discovered in previous studies and suggested to be a result of various demographic and 596 selection processes $(35,36)$. To keep our methodology consistent throughout our 597 analyses, we estimated the TMRCA of African and non-African $Y$ chromosomes as the 598 length of the non-African $Y$ lineage (sum of branch lengths $a+d$ in Figure S7.1). 599 Encouragingly, we found that our mutation rate and TMRCA $A F R$ point estimates (Table 600 S7.2) match closely those based on a large panel of present-day $Y$ chromosomes (21). 601 Most importantly, using the A00 lineage as a representative of the deepest known split 
602 among present-day human $Y$ chromosomes we inferred a TMRCA $A 00$ of $\sim 249$ years ago 603 (point estimate based on an estimated mutation rate of $7.34 \times 10^{-10}$ per bp per year), which 604 is comparable to the TMRCA $A 00$ of $\sim 254$ years ago estimated by Karmin et al., 2015. 605 Therefore, our more restricted 6.9 Mb capture target gives TMRCA estimates consistent 606 with those obtained from the full $Y$ chromosome shotgun data (21). 


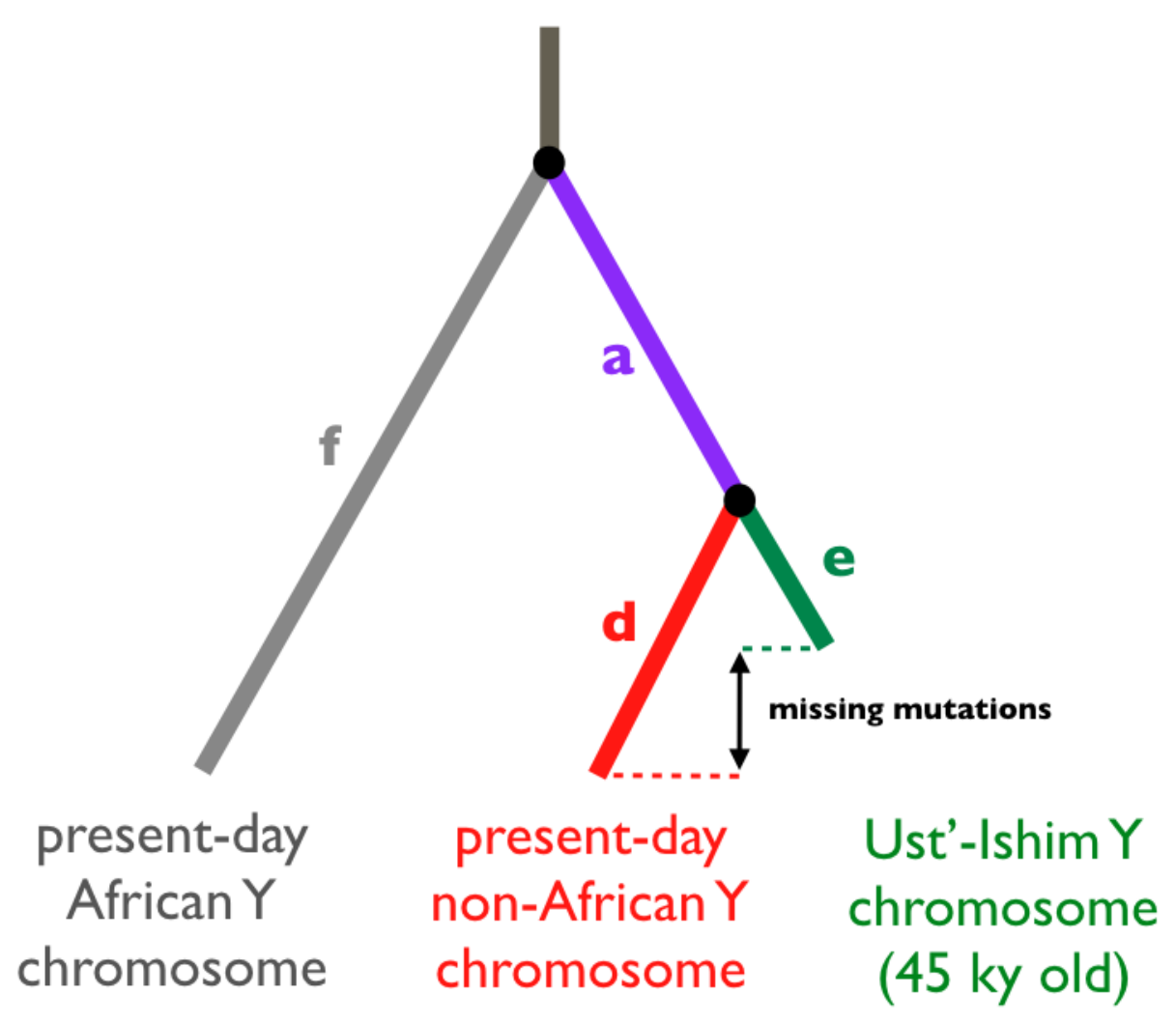

610 Figure S7.1. Schematic of the branch-counting method to estimate the mutation

611 rate and split times of African and non-African Y chromosomes. Accurate knowledge

612 of the age of the Ust'-Ishim individual (22) makes it possible to estimate the mutation rate

613 within the $6.9 \mathrm{Mb}$ target capture regions. We use the number of mutations missing on the

614 Ust'-Ishim lineage since this individual died (45 kya) and compare it to another non-

615 African $\mathrm{Y}$ chromosome, i.e. the quantity $d-e$. We used this mutation rate to calculate the

616 TMRCA between a pair of non-African and African $Y$ chromosomes as the total length of

617 the branches $a+d$. 


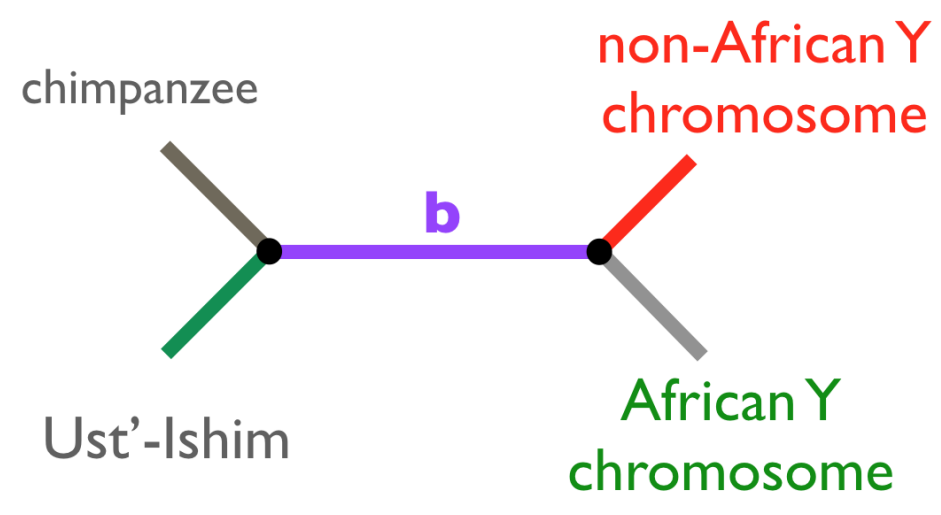

618

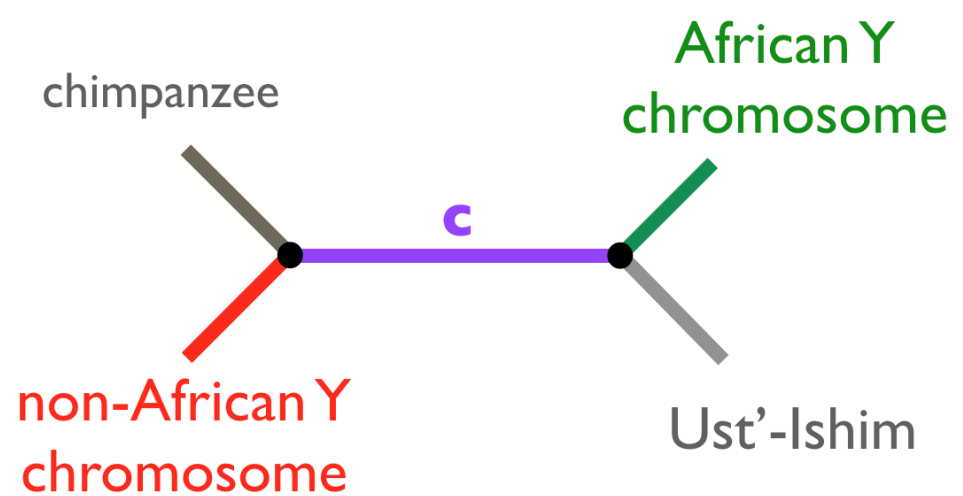

619 Figure S7.2. Two alternative site patterns which are discordant with the true

620 phylogenetic relationship. Given that the true phylogenetic relationship is that shown in

621 Figure S7.1, these alternative branch patterns must be a result of double mutations or 622 genotype calling errors.

623 


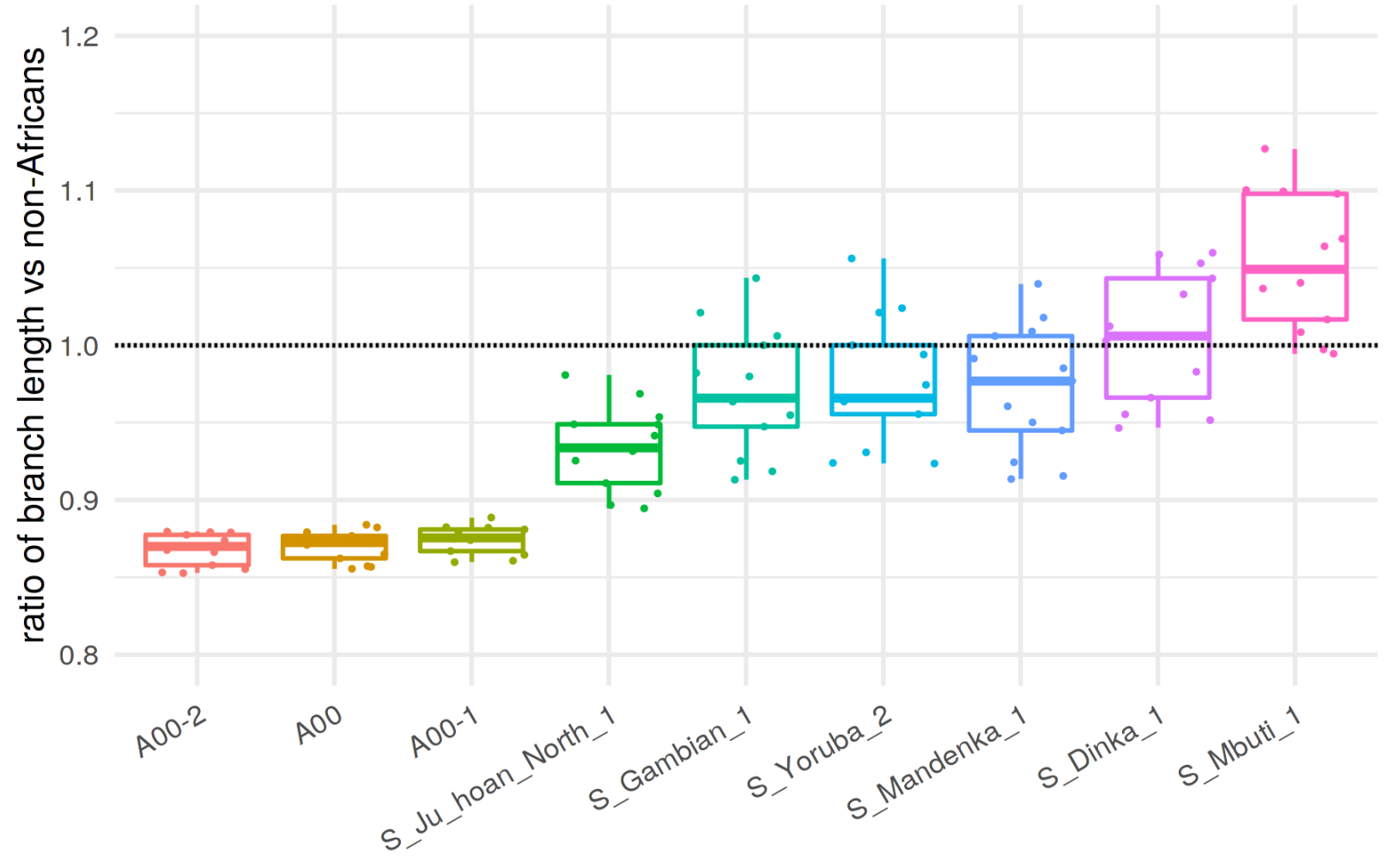

African $Y$ chromosome

626 Figure S7.3. Branch length differences between African $Y$ chromosomes and a

627 panel of 13 non-African $Y$ chromosomes. Ratios were calculated by creating an

628 alignment of chimpanzee, African and non-African $Y$ chromosomes and taking the ratio

629 of the number of derived alleles observed in an African (x-axis) and the number of derived

630 alleles in each of the individual non-Africans (dots, Table S7.1). "A00" represents a merge

631 of sequences of two lower coverage $Y$ chromosomes, A00-1 and A00-2 (Table S4.3). 


\begin{tabular}{|l|l|}
\hline individual & haplogroup \\
\hline S_Finnish-2 & I1a1b5 \\
\hline S_Sardinian-1 & J1b2b \\
\hline S_BedouinB-1 & J1b2b \\
\hline S_Punjabi-1 & J2a1 \\
\hline S_French-1 & J2a1b1 \\
\hline S_Turkish-1 & R1b1a2a1a2c1g \\
\hline S_Karitiana-1 & Q1a2a1a1 \\
\hline S_Saami-2 & N1c1a1a2a \\
\hline S_Burmese-1 & O3a1c2 \\
\hline S_Thai-1 & O3a2c \\
\hline S_Dai-2 & O2a1 \\
\hline S_Han-2 & O1a1 \\
\hline S_Papuan-2 & S \\
\hline S_Dinka-1 & E2a \\
\hline S_Gambian-1 & E1b1a1a1f \\
\hline S_Ju_hoan_North-1 & B2b1b \\
\hline S_Mandenka-1 & E1b1a1a1g1 \\
\hline S_Mbuti-1 & E1b1a1a1g1 \\
\hline S_Yoruba-2 & E1b1a1a1f1b1 \\
\hline A00 & A00 \\
\hline A00-1 & A00 \\
\hline A00-2 & A00 \\
\hline
\end{tabular}

Table S7.1. Haplogroups of present-day human $Y$ chromosomes used in our

637 reference panel. Haplogroup names were taken from the SGDP annotation table (20).

638 Haplogroups of the African panel are highlighted in gray. The A00 individual represents

639 a merge of lower coverage sequences of two individuals, here named A00-1 and A00-2

640 (Table S4.3).

641

642 


\begin{tabular}{|c|c|c|c|c|c|c|c|c|c|}
\hline African & a & b & c & d & e & f & total & $\begin{array}{l}\text { mutation rate } \\
\left(\text { bp }^{-1} \times \text { year }^{-1}\right)\end{array}$ & $\begin{array}{r}\text { TMRCA AFR } \\
\text { (years ago) }\end{array}$ \\
\hline $\mathrm{A} 00$ & 1060.2 & 1.2 & 8.3 & 214.5 & 14.2 & 1103.1 & $6,064,477$ & $7.34 \mathrm{e}-10$ & 249211.63 \\
\hline $\mathrm{A} 00-2$ & 1054.5 & 1.2 & 8.2 & 214.7 & 14.6 & 1092.7 & $6,045,632$ & $7.35 e-10$ & 247074.54 \\
\hline$A 00-1$ & 1004.2 & 1.2 & 8.1 & 207.2 & 14.2 & 1054.1 & $5,855,414$ & $7.32 \mathrm{e}-10$ & 246756.41 \\
\hline S_Ju_hoan_North_1 & 261.5 & 2.7 & 8.5 & 216.3 & 14.5 & 439.2 & $6,100,839$ & $7.35 e-10$ & 98443.62 \\
\hline S_Mbuti_1 & 116.5 & 1.2 & 8.9 & 217.5 & 14.5 & 344.7 & $6,123,504$ & $7.37 e-10$ & 76782.21 \\
\hline S_Dinka_1 & 110.8 & 2.1 & 8.7 & 217.4 & 14.5 & 321.2 & $6,090,467$ & $7.40 \mathrm{e}-10$ & 71573.49 \\
\hline S_Mandenka_1 & 120.9 & 1.2 & 8.8 & 218.3 & 14.5 & 321.0 & $6,123,094$ & $7.39 \mathrm{e}-10$ & 71226.46 \\
\hline S_Gambian_1 & 118.8 & 1.2 & 8.5 & 218.1 & 14.5 & 320.2 & $6,096,582$ & $7.42 \mathrm{e}-10$ & 71127.40 \\
\hline S_Yoruba_2 & 118.9 & 1.2 & 8.7 & 217.6 & 13.5 & 320.9 & $6,121,558$ & $7.41 \mathrm{e}-10$ & 71119.46 \\
\hline
\end{tabular}

645 of non-Africans calculated from the length of the $a+d$ branch as shown in Figure S7.1. 


\subsection{Archaic human-modern human TMRCA (TMRCA archaic)}

659

Having estimated $T M R C A_{A F R}$ (section 7.1), we can express the TMRCA of archaic and modern human $\mathrm{Y}$ chromosomes (TMRCA archaic $)$ as a factor of how much older is $T M R C A_{\text {archaic }}$ compared to $T M R C A_{A F R}$ (Figure S7.4). In mathematical terms, if we call the scaling factor $\alpha$ (following the terminology of (5)), we can write

$$
T M R C A_{\text {archaic }}=\alpha \times T M R C A_{A F R} .
$$

665

In the remainder of this section, we present two ways of calculating $\alpha$, first using the original approach of Mendez et al. and then using a more straightforward method.

\section{Mendez et al. approach to calculate $\alpha$}

671 Based on Figure S7.4, an alternative way to express $T M R C A_{\text {archaic }}$ in addition to 672 equation (1) is

$$
T M R C A_{\text {archaic }}=T_{\text {shared }}+T M R C A_{A F R}
$$

675 The expressions (1) and (2) define a system of two equations and three variables, which can be solved for $T_{\text {shared }}$ to get

$$
T_{\text {shared }}=\alpha \times T M R C A_{A F R}-T M R C A_{A F R}=T M R C A_{A F R} \times(\alpha-1) .
$$


681 Mendez et al. found an expression for $\alpha$ by considering a ratio of time shared by hg19 682 and $\mathrm{A} 00 \mathrm{Y}$ chromosomes after their split from the El Sidrón 1253 Neandertal $\left(T_{\text {shared }}\right.$ 683 above) and private branch lengths of both (Figure S7.4), arriving at the following expression:

685

$$
\frac{T_{a}}{T_{a}+T_{d}+T_{e}}=\frac{T_{\text {shared }}}{T_{\text {shared }}+2 T_{A F R}}=\frac{T_{A F R}(\alpha-1)}{T_{A F R}(\alpha-1)+2 T_{A F R}}=\frac{\alpha-1}{\alpha+1} .
$$

686

Assuming mutation rate constancy on different lineages, $\alpha$ can be found by solving the following equation

which leads to

$$
\frac{a}{a+d+e}=\frac{\alpha-1}{\alpha+1}
$$

$$
\alpha=\frac{2 a+d+e}{d+e}
$$

692

693 Using this expression for $\alpha$ and the values of $T M R C A_{A F R}$ estimated in section 7.1, we can 694 calculate $T M R C A_{\text {archaic }}$ for each pair of archaic and non-African $Y$ chromosomes using 695 equation (5) (Figure 7.9B).

698 While investigating the effect of minimum coverage filtering on genotype calling accuracy 699 (section 5.3), we discovered a concerning dependence of the apparent branch lengths on 700 the choice of the minimum coverage cutoff. Under normal conditions, the relative 701 proportions of branch lengths $a, d$ and $e$ (Figure S7.4) should remain constant regardless 702 of coverage. This is crucial because the $\alpha$ estimator proposed by Mendez et al. is 703 expressed in terms of proportions of lengths of all three of these branches (equation (5)). 
Strikingly, we found that although the proportions of $a$ and $d$ branch lengths remain relatively stable even for extremely strict coverage filters, the relative length of the $e$ branch (given by the proportion of derived mutations on the private African branch) has increasing tendency (Figures S7.6 and S7.7). Furthermore, although this effect is most pronounced in low coverage samples (Figures S7.6 and S7.7), it is clearly present even in the high coverage Mezmaiskaya 2, although at much higher coverage cutoffs (Figure S7.8). Therefore, the issue is clearly not sample-specific but is a common artifact caused by pushing the minimum required coverage close to, or even beyond, the average coverage. Restricting to sites with high number of aligned reads leads to enrichment of

713 regions of lower divergence from the reference sequence, distorting the normal proportions of derived mutations observed on different branches of the tree.

716 We note that there is a more straightforward way to express the scaling factor $\alpha$ :

$$
\alpha=\frac{a+d}{d} \text {. }
$$

718 This follows trivially from the definition of $\alpha$ as the factor of how much deeper

$719 T M R C A_{\text {archaic }}$ is compared to $T M R C A_{A F R}$ and, unlike the original formulation of $\alpha$

720 (equation (5)), has the advantage of not relying on the relative length of the African branch

$721 e$. This is important not only because of discordant branch proportion patterns (Figures

722 S7.6-S7.8) but also due to known unequal branch lengths observed in African and non-

723 African Y chromosome lineages (Figure S7.3, (36, 37)).

724

725 For completeness, we note that in a situation without any bias we can assume $e \approx d$.

726 Substituting for $e$ in equation (5) then gives 


$$
\alpha=\frac{2 a+d+e}{d+e} \approx \frac{2 a+2 d}{2 d} \approx \frac{a+d}{d}
$$

729 Therefore, under ideal conditions, both approaches to calculate $\alpha$ (equations (5) and (6))

730 are mathematically equivalent.

732 Using the new expression for $\alpha$ and the values of $T M R C A_{A F R}$ estimated in section 7.1, we

733 can estimate $T M R C A_{\text {archaic }}$ for each pair of archaic and non-African $Y$ chromosomes

734 using equation (5) (Figure 2A, Table S7.3). By comparing TMRCA results based on the

735 two formulations for $\alpha$, we found similar estimates for most of the archaic human $Y$

736 chromosomes in our study (Figures S7.9 and S7.10). The only exception are the two

737 Denisovan $\mathrm{Y}$ chromosomes, for which we infer slightly higher TMRCA with modern

738 humans using the new $\alpha$ estimation procedure compared to the formulation based on the

739 original method (Figures S7.9 and S7.10). This is a consequence of an increased

740 proportion of the $e$ branch relative to the $d$ branch in Denisova 4 and Denisova 8 at the

741 chosen minimum coverage filter which is evident in Figure S7.7. Because the original

742 method of Mendez et al. relies on the $e$ count of the derived African alleles (equation (5)),

743 this leads to a slight decrease in the value of the $\alpha$ factor and, consequently, to a lower

744 inferred TMRCA value. In contrast, our new formulation of $\alpha$ (equation (6)) is robust to

745 this artifact and the inferred values of TMRCA are not affected.

747 Finally, we want to emphasize that although the analyses of branch length discrepancies

748 discussed in this section were mostly based on results obtained using the A00 Y 749 chromosome lineage, the issues we discovered are not specific to a particular choice of 

an African Y chromosome (Figure S7.8A-C). However, comparisons of TMRCA estimates

751 for the low coverage samples with those obtained for the high coverage samples (which

752 do not show any biases at coverage cutoffs used throughout our study) clearly show that

753 the inferences are most stable when the A00 lineage is used in the calculation of the $\alpha$

754 scaling factor, even for the samples with lowest coverage (Figure S7.13). Therefore, all

755 main results in our study are based on calculations using the A00 high coverage $Y$ 756 chromosome. 


\section{chimpanzee}

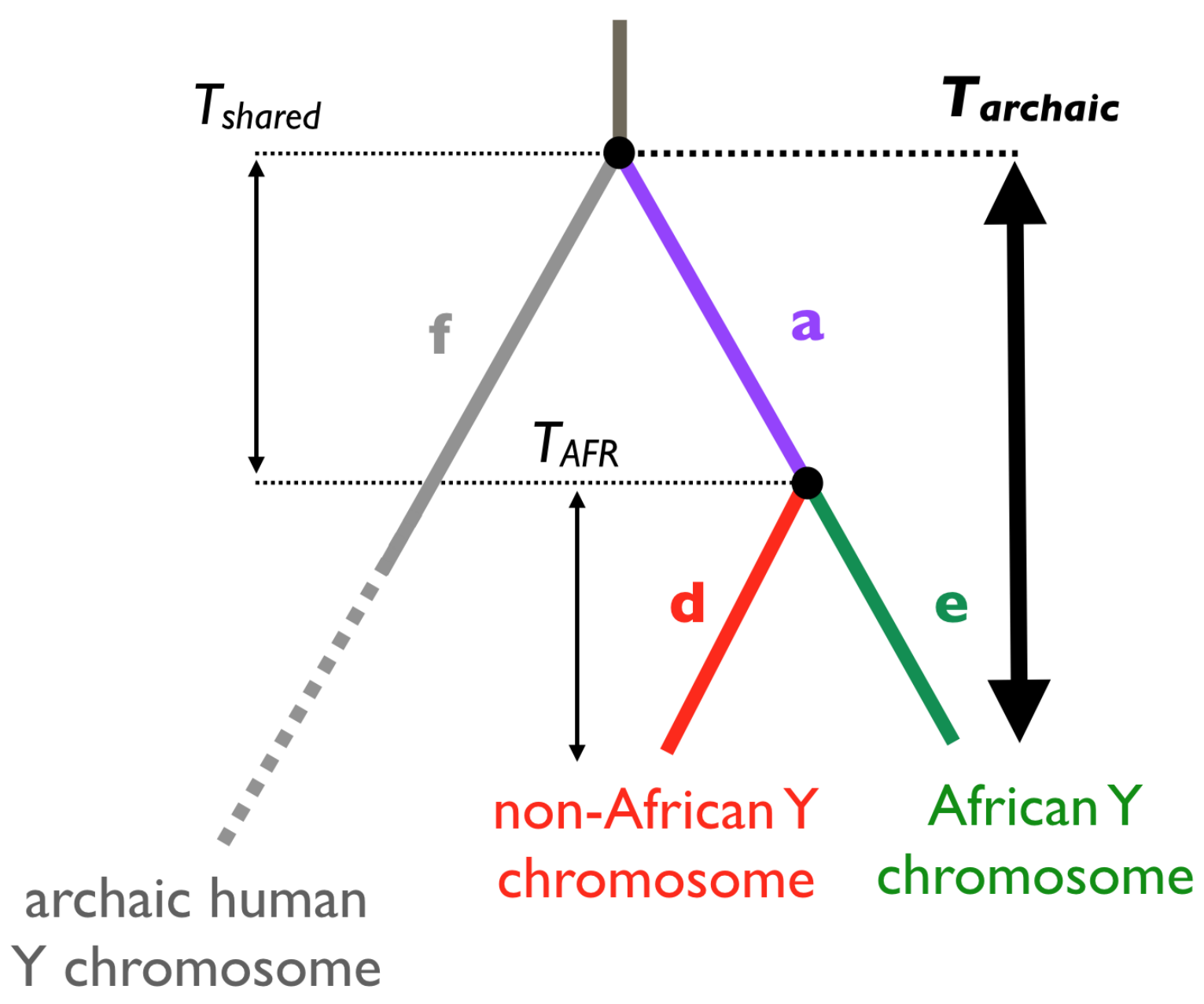

Figure S7.4. Branch-counting method to estimate the TMRCA of archaic and present-day human Y chromosomes. As explained in section 7.2, we can decompose

762 the quantity of interest $\left(T_{A R C H}\right.$, thick arrow) using two quantities $T_{\text {shared }}$ and $T_{A F R}$ and 763 express it simply as a factor of $T_{A F R}$, which can be accurately estimated using high-quality modern human $\mathrm{Y}$ chromosome sequences (section 7.1). 

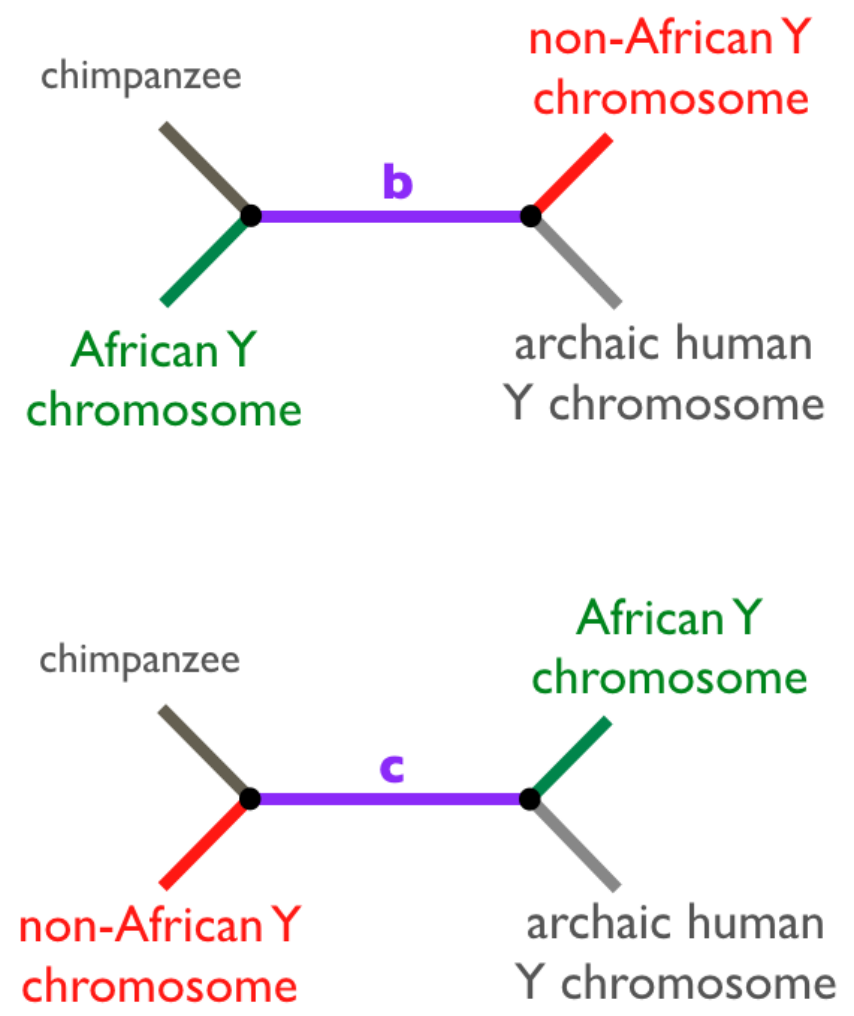

768 Figure S7.5. Alternative tree topologies with two additional possible branch counts

$769 \boldsymbol{b}$ and $\boldsymbol{c}$. These topologies are incongruent with the true phylogenetic trees and the branch

770 counts $b$ and $c$ are most likely a result of back mutations or sequencing errors. 
A

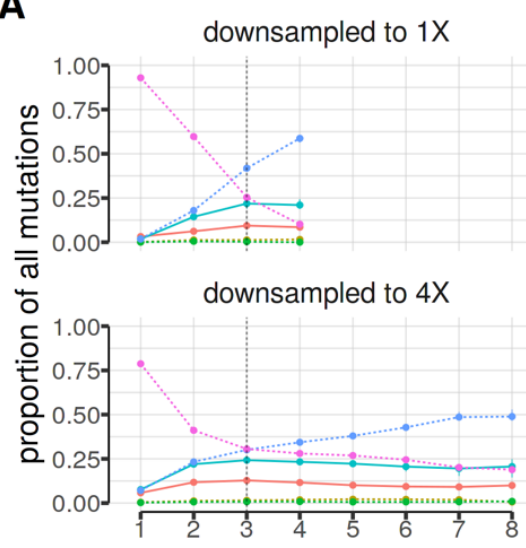

\section{B}

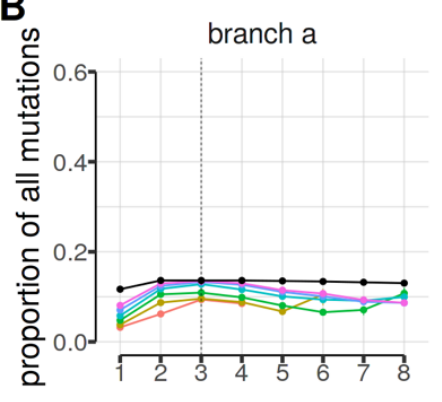

downsampled to $2 \mathrm{X}$

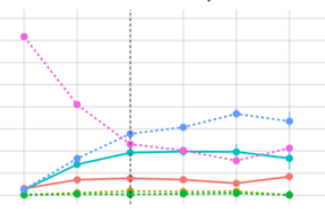

downsampled to $5 \mathrm{X}$

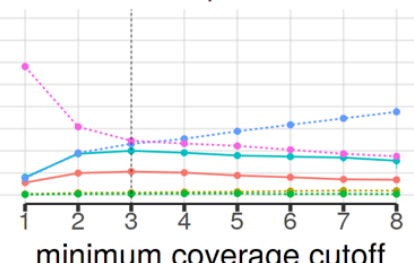

downsampled to $3 X$

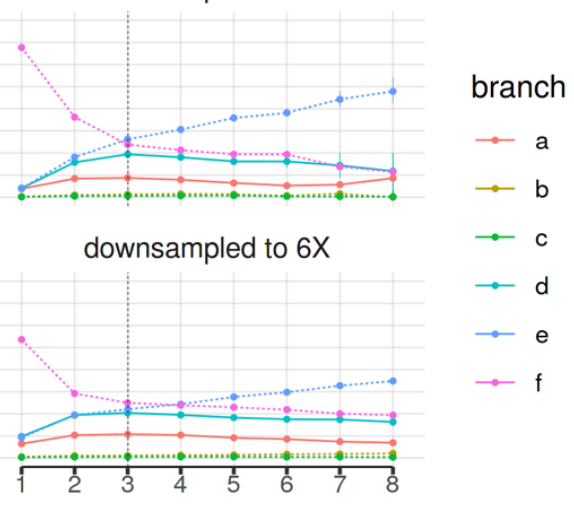

$\rightarrow$ downsampled to $1 \mathrm{X} \rightarrow$ downsampled to $3 \mathrm{X} \rightarrow$ downsampled to $5 \mathrm{X} \rightarrow$ Mezmaiskaya 2

$\rightarrow$ downsampled to $2 \mathrm{X} \rightarrow$ downsampled to $4 \mathrm{X} \rightarrow$ downsampled to $6 \mathrm{X}$

branch d

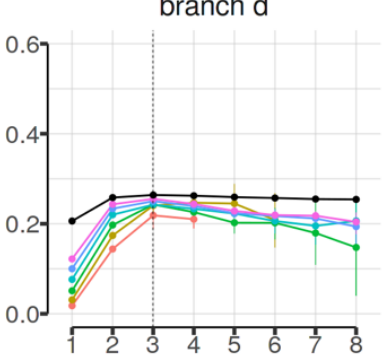

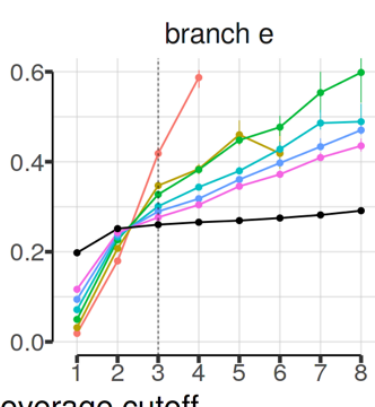

branch e

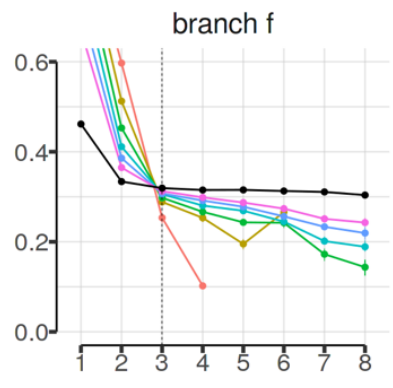

minimum coverage cutoff

775 Figure S7.6. Relative proportion of branch lengths in downsampled Mezmaiskaya

778 but partitioned per branch. Black solid lines show expectations based on the full

779 Mezmaiskaya 2 data. Increased relative proportions of the $f$ branch lengths are due to

780 false polymorphisms at low coverage cutoffs. Branch length proportions (labeled as in

781 Figure S7.4) were calculated as $\frac{a}{N}, \frac{b}{N}, \ldots, \frac{f}{N}$, where $N=a+b+\cdots+f$. Vertical dotted lines

782 indicate a $3 \mathrm{X}$ lower coverage cutoff used throughout our study (section 5.3). Branch

783 counts were averaged over pairs of 13 non-Africans and the A00 African $\mathrm{Y}$ chromosome.

784 Analysis is based on all classes of polymorphisms. 


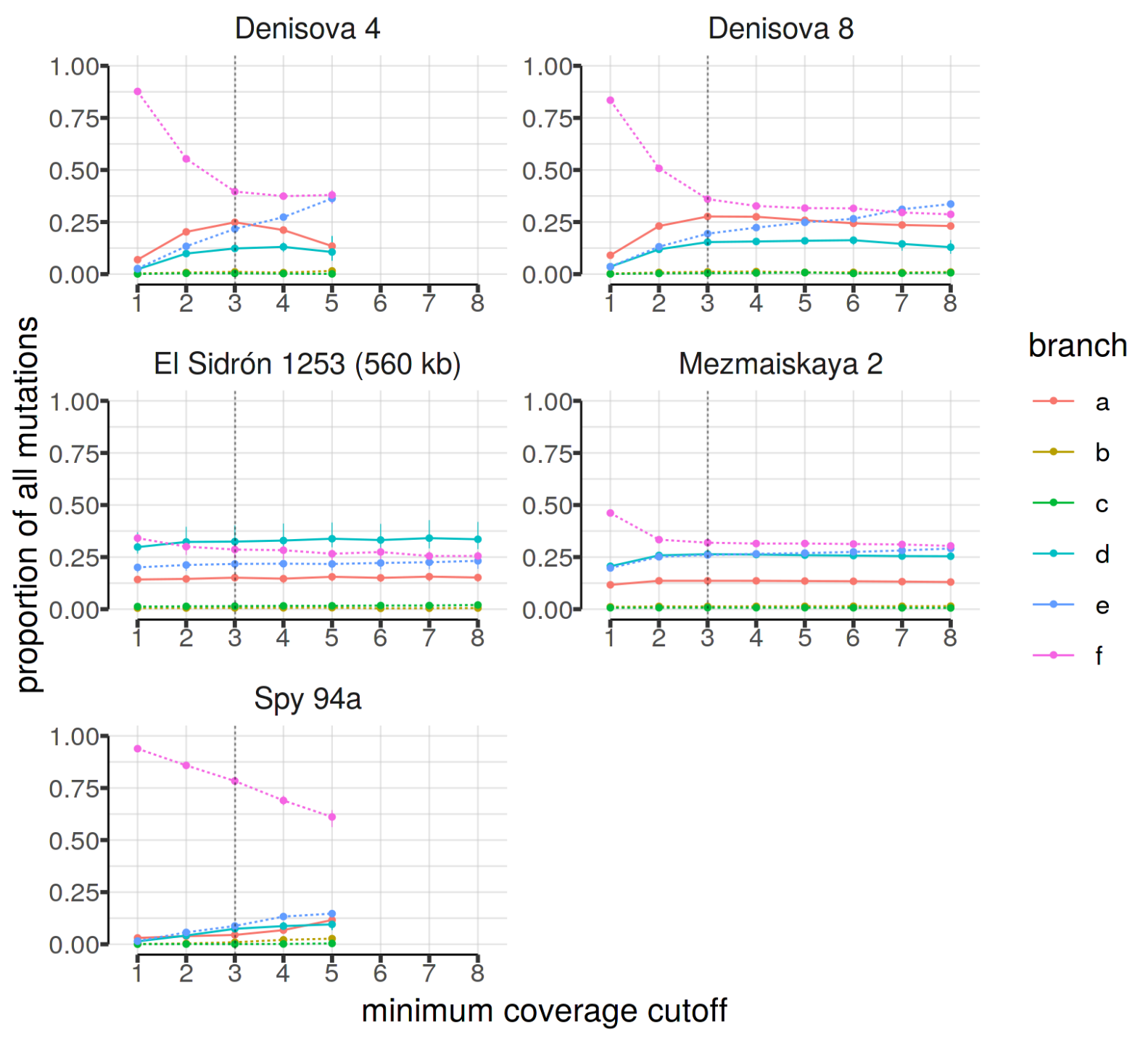

787 Figure S7.7. Relative proportion of branch lengths as a function of minimum

788 coverage support required for each genotype call. Branch length proportions (colored

789 lines) were calculated as $\frac{a}{N}, \frac{b}{N}, \ldots, \frac{f}{N}$, where $N=a+b+\cdots+f$. Vertical dotted lines

790 indicate a $3 X$ lower coverage cutoff used throughout our study (section 5.3). With the 791 exception of El Sidrón 1253, all panels show results for the 6.9 Mb capture data. Analysis 792 is based on all polymorphisms. 

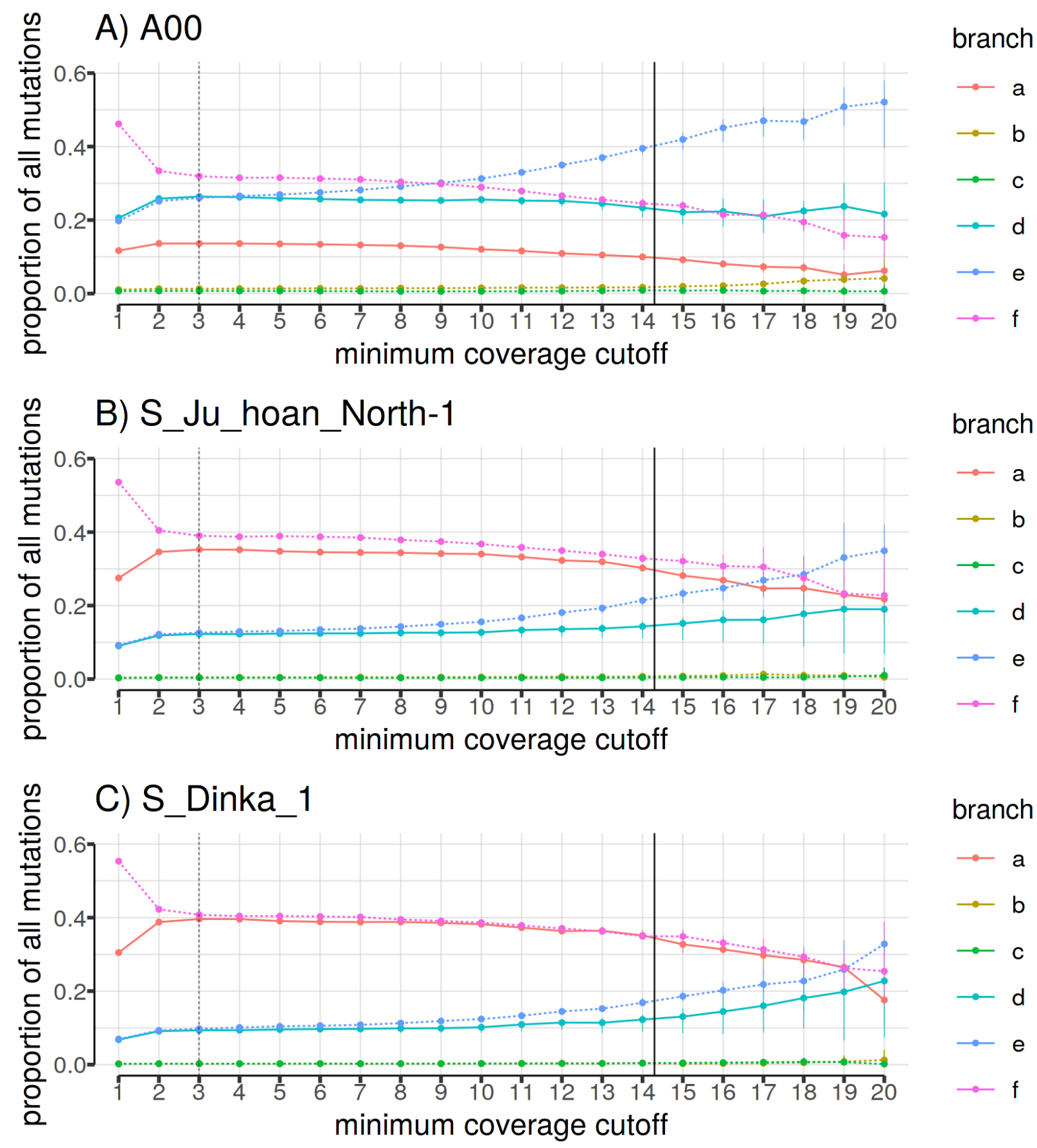

Figure S7.8. Relative proportions of branch lengths in the 14.3X Mezmaiskaya 2 
800 show results based on three different African $Y$ chromosomes used to define branch $e$

801 (Figure S7.4). Branch length proportions (colored lines) were calculated as $\frac{a}{N}, \frac{b}{N}, \ldots, \frac{f}{N}$,

802 where $N=a+b+\cdots+f$, using the complete $6.9 \mathrm{Mb}$ capture data of Mezmaiskaya 2.

803 Vertical dotted lines indicate a $3 X$ lower coverage cutoff used throughout our study

804 (section 5.3). Analysis is based on all polymorphisms. Vertical solid lines indicate the 805 average coverage in the Mezmaiskaya 2 individual (14.3X).

806 


\section{A) New TMRCA statistic}

all SNPs

excluding C-T and G-A
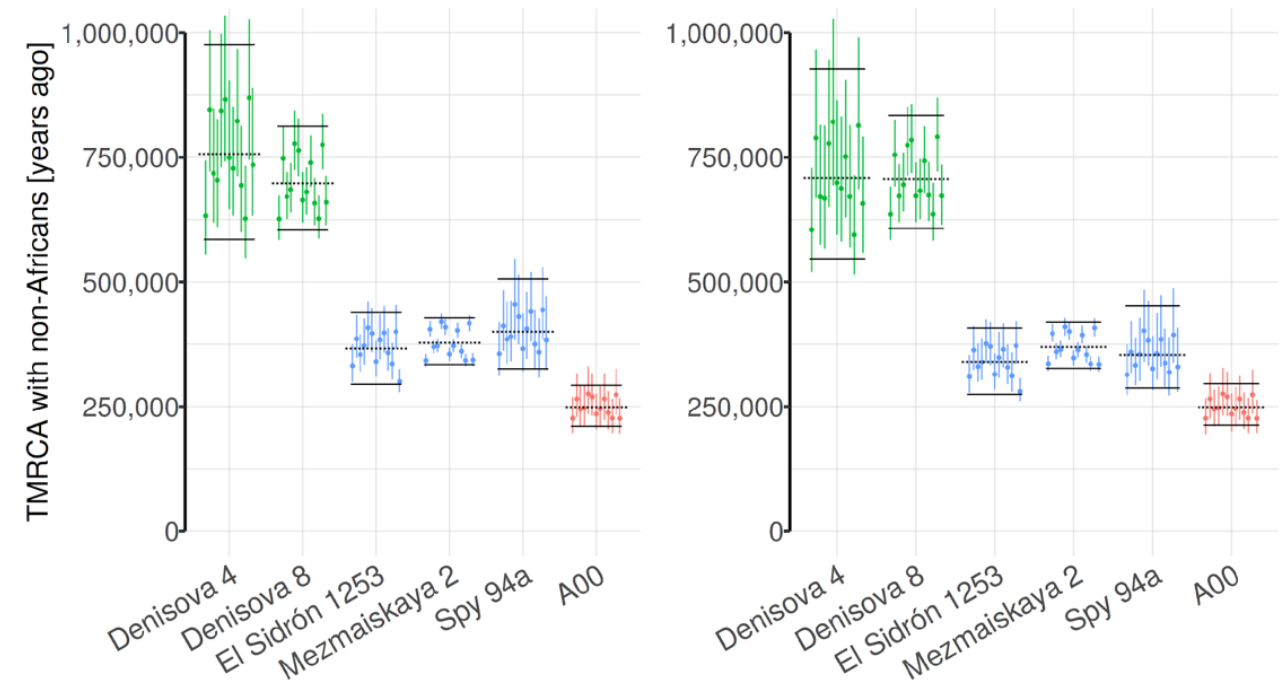

B) TMRCA statistic of Mendez et al.

all SNPS
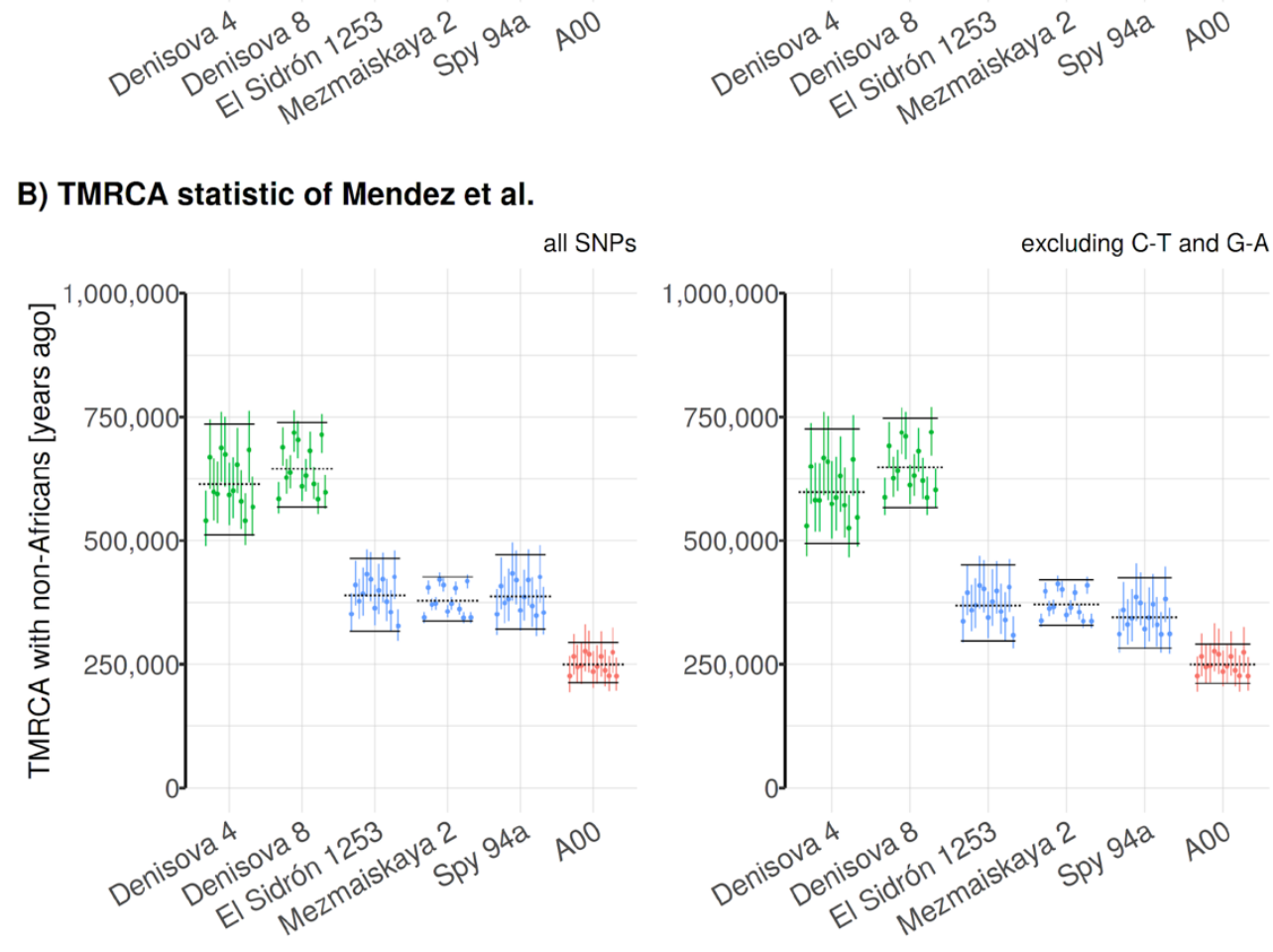

808 Figure S7.9. Comparison of TMRCA estimates obtained using the new statistic and

809 the original approach used in the analysis of the El Sidrón 1253 Neandertal. (A)

810 Estimates using our new, more robust TMRCA estimate. (B) Original calculation

811 proposed by Mendez et al., 2016 (5). Shown are estimates based on all polymorphisms

812 (left panels) and excluding C-to-T or G-to-A polymorphisms which are more likely to be

813 caused by aDNA damage (right panels). 


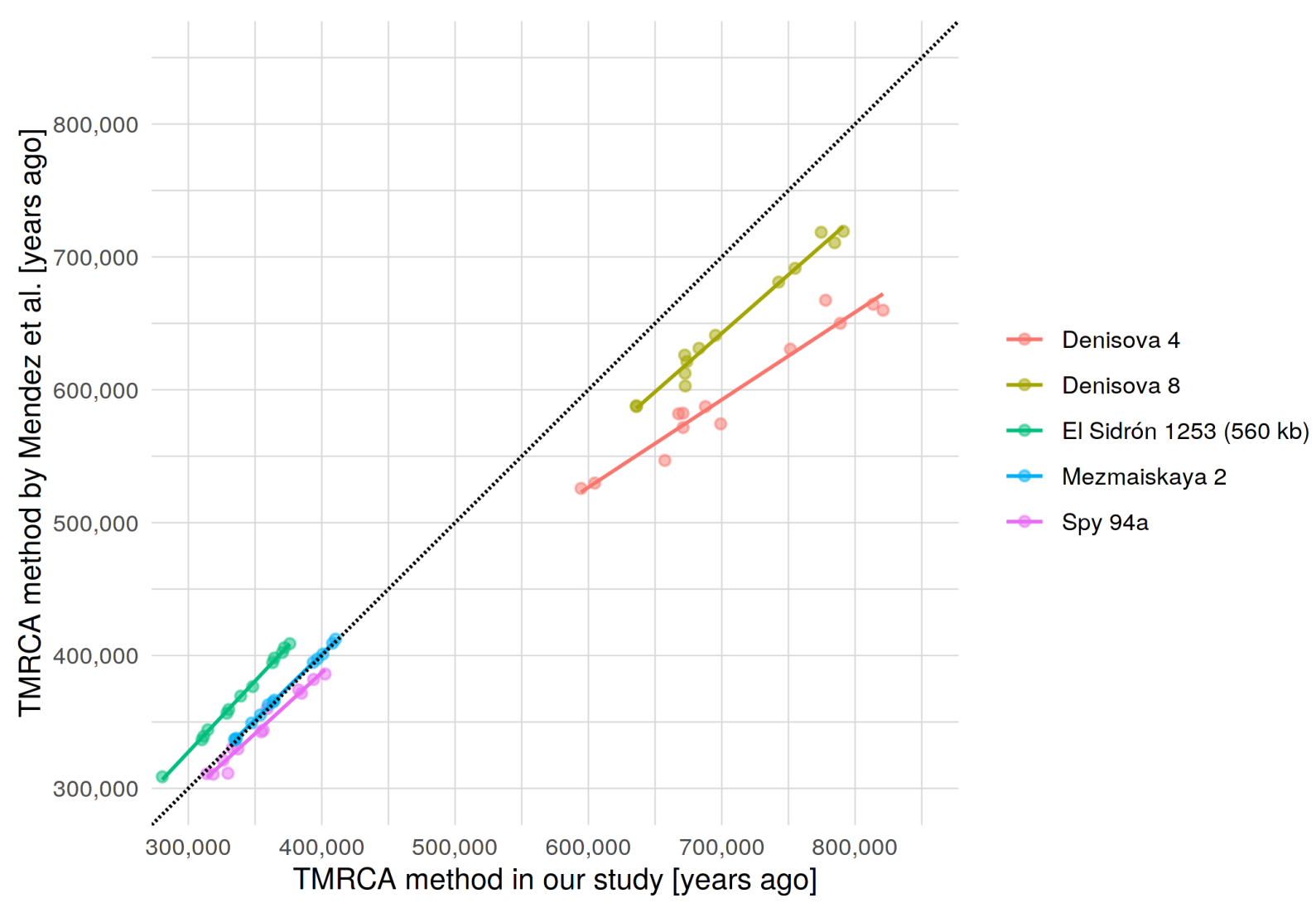

815 Figure S7.10. Comparison of TMRCA estimates obtained using a new statistic and

816 the original approach used in the analysis of the El Sidrón 1253 Neandertal. This

817 figure shows the same data as in Figure S7.9 (panels on the left) but plotted on the same

818 scatterplot for easier comparison. Dotted black line shows the the line of perfect

819 correlation. The TMRCA between the Denisovan individuals and modern human is slightly

820 underestimated due to a bias in the TMRCA estimation procedure proposed in the original

821 study of the El Sidrón 1253 Neanderthal (5). Analysis is based on all polymorphisms

822 except C-to-T and G-to-A changes.

823

824 


\section{A) $\mathbf{5 0 \%}$ mappability filter}

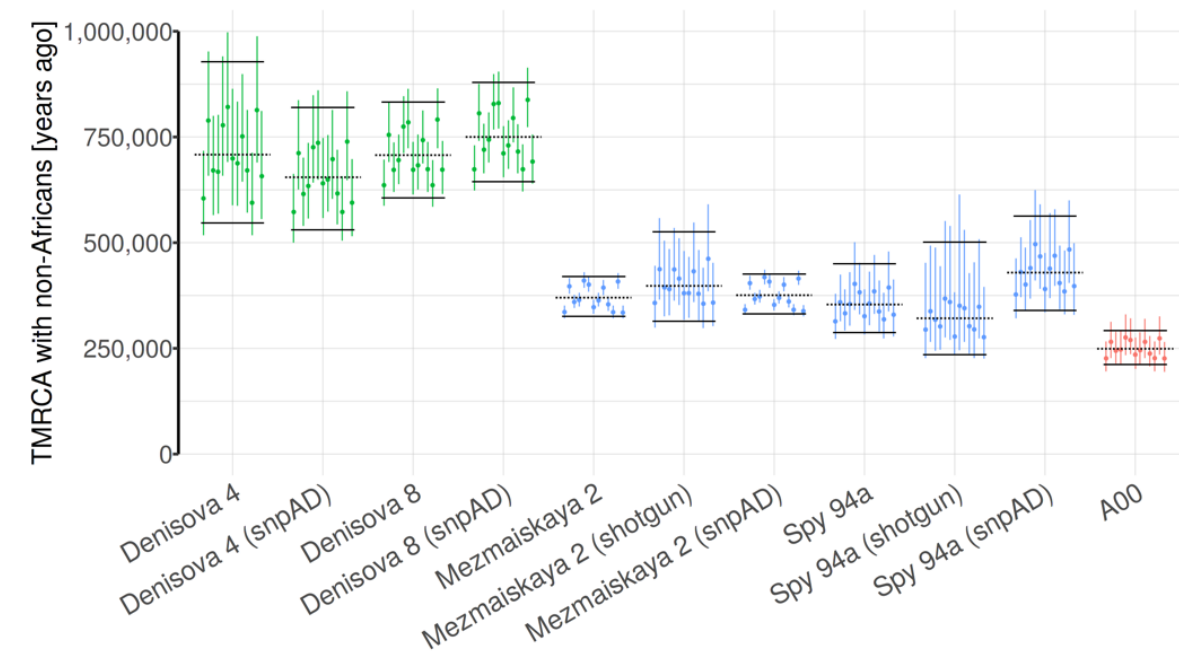

B) $100 \%$ mappability filter

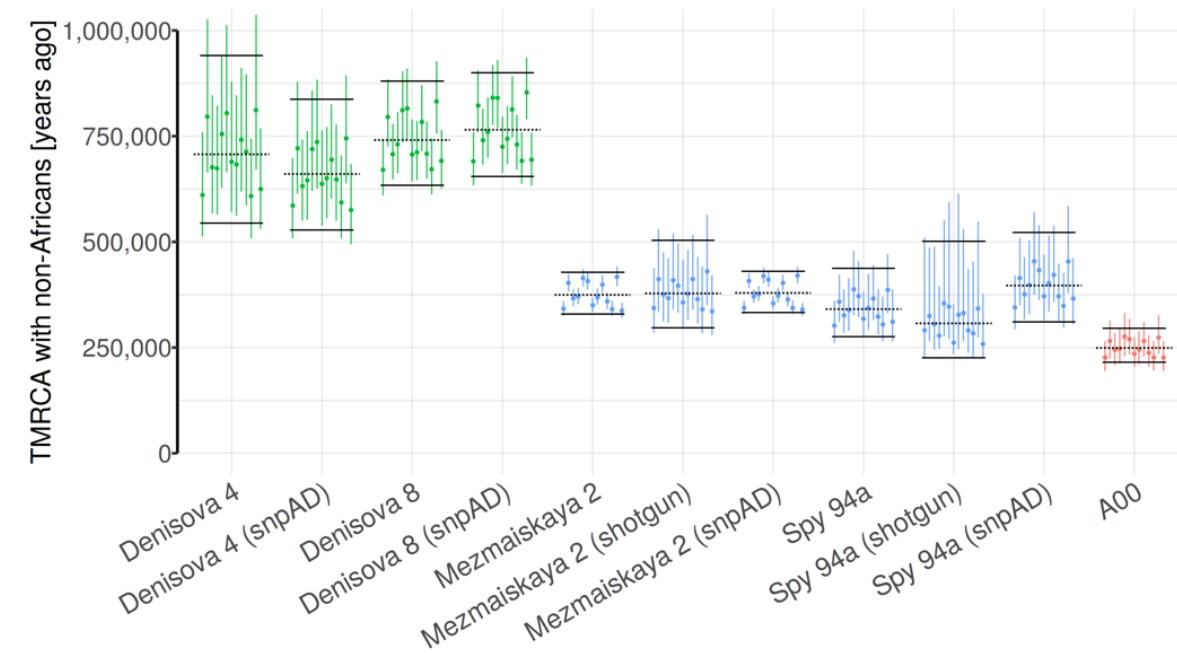

826 Figure S7.11. Detailed evaluation for potential technical biases in our TMRCA

827 estimates. Shown are TMRCA results based on different versions of the data (shotgun 828 or capture) or genotype calling methods (snpAD or consensus-based genotype calling 829 method - which is the default). Panels (A) and (B) show results based on two versions of 830 mappability filters - less strict ("map35_50\%") and more strict ("map35_100\%”) filters 831 described in the Altai Neandertal study (2). Analysis is based on all polymorphisms except 832 C-to-T and G-to-A changes. 


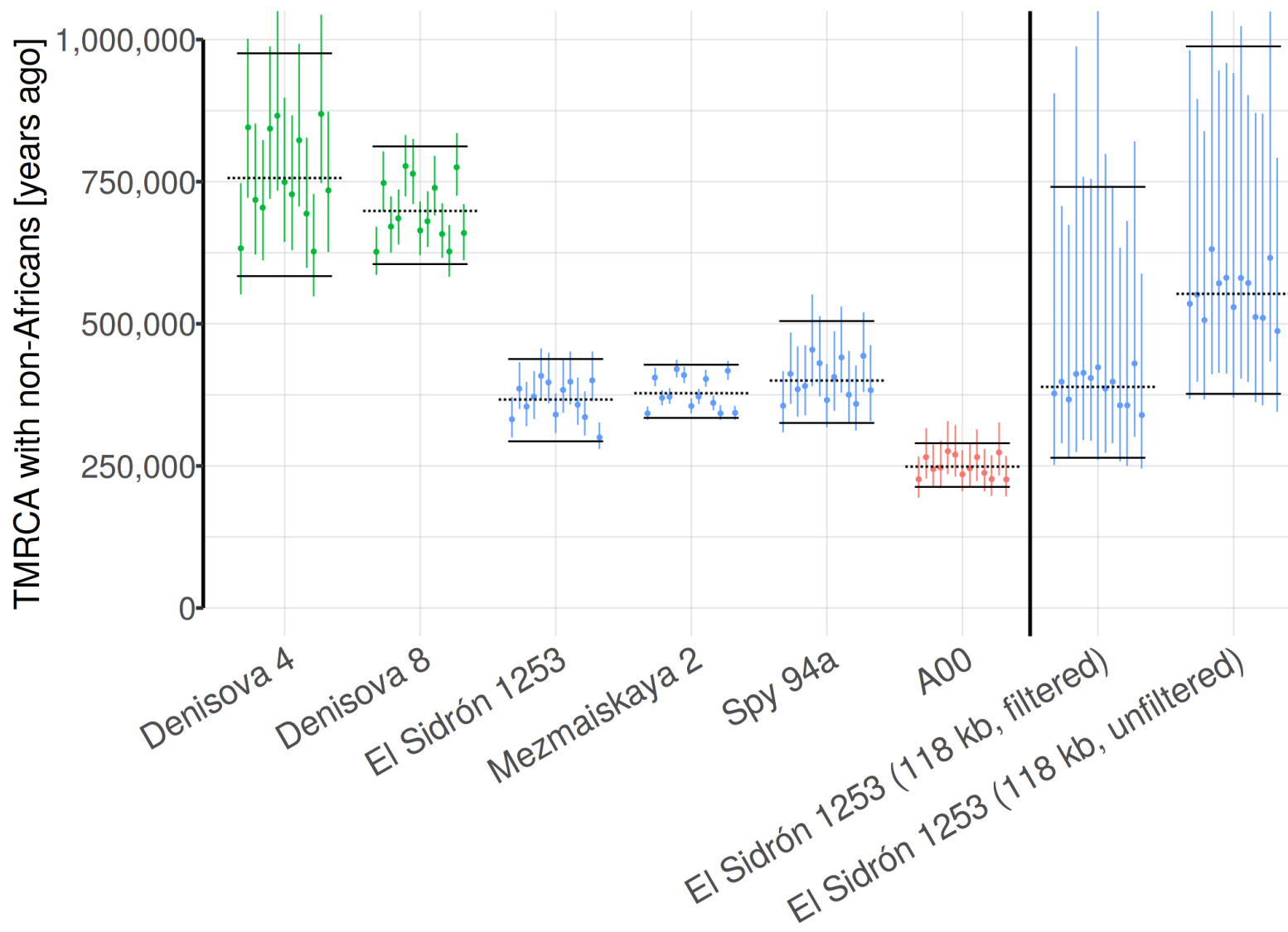

834 Figure S7.12. TMRCA between the El Sidrón 1253 and modern human Y chromosomes. TMRCA estimates obtained for new capture sequence from the El Sidrón 1253 Neanderthal ( 370 kya) differ significantly from the previously published results based on the $118 \mathrm{~kb}$ of the coding capture sequence ( 600 kya, "118 kb, unfiltered"

838 column on the right from the vertical line). We found that applying stricter filtering criteria 839 results in the same TMRCA values we obtain for the new capture data ("118 kb, filtered" 840 column on the right from the vertical line). Analysis is based on all polymorphisms. 

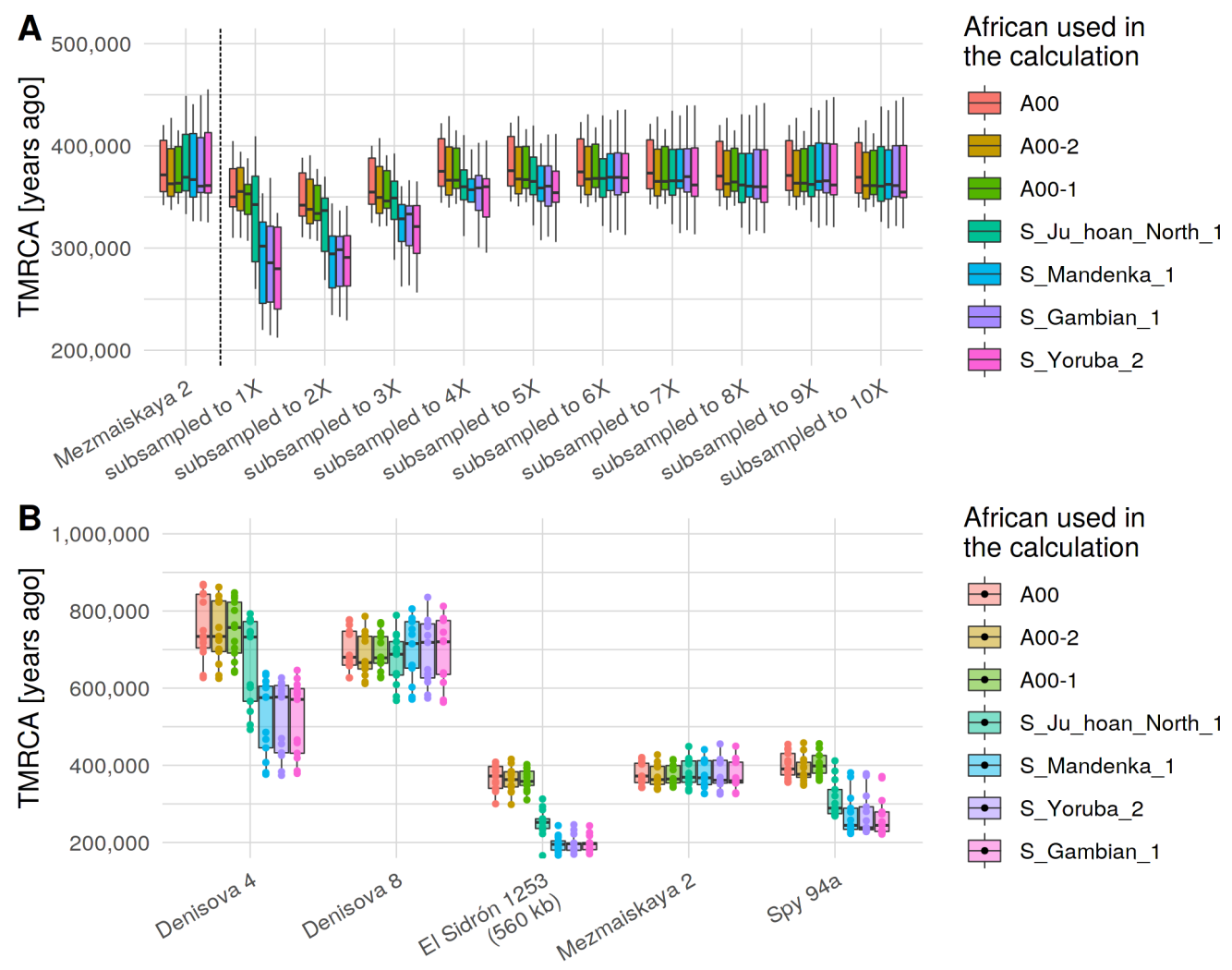

844 Figure S7.13. Estimates of $T M R C A_{\text {archaic }}$ for different African $\mathrm{Y}$ chromosomes used

845 in the calculation. (A) Results for the high-coverage 14.3X Mezmaiskaya 2 capture data

846 (left of the vertical line) and its subsets generated by downsampling. A00-based TMRCA

847 estimates are quite stable across the entire range of coverage and match those for the

848 full data. In contrast, estimates based on other, less divergent, African Y chromosomes

849 are heavily biased, and this bias is especially strong for low coverage samples. (B) A00-

850 based estimates for Denisova 4, El Sidrón 1253 and Spy 94a match those for their higher

851 coverage counterparts (Denisova 8 and Mezmaiskaya 2, respectively) as is required by

852 the topology of the phylogenetic tree (Figure 2A). However, estimates based on other

853 African individuals show the same bias shown for low coverage samples in panel (A).

854 Dots show TMRCA estimates based on 13 non-African individuals. Both analyses are

855 based on all polymorphisms. 


\begin{tabular}{|r|c|c|c|c|c|c|c|c|c|c|}
\hline name & a & $\mathbf{b}$ & $\mathbf{c}$ & $\mathbf{d}$ & $\mathbf{e}$ & $\mathbf{f}$ & total & $\begin{array}{c}\text { TMRCA } \\
\text { [years } \\
\text { ago] }\end{array}$ & $\begin{array}{c}\text { TMRCA } \\
\text { (lower Cl) }\end{array}$ & $\begin{array}{c}\text { TMRCA } \\
\text { (upper } \\
\text { Cl) }\end{array}$ \\
\hline Denisova 4 & 142.2 & 2.0 & 2.2 & 77.8 & 125.8 & 165.9 & $1,084,363.6$ & $708,133.1$ & $549,422.5$ & $930,979.7$ \\
\hline Denisova 8 & 583.5 & 8.8 & 8.5 & 318.0 & 410.4 & 583.9 & $3,372,262.6$ & $706,874.9$ & $607,187.2$ & $833,211.4$ \\
\hline Mezmaiskaya 2 & 301.8 & 13.8 & 16.4 & 625.1 & 609.1 & 651.9 & $5,349,303.5$ & $369,637.7$ & $326,137.1$ & $419,311.0$ \\
\hline Spy 94a & 17.4 & 4.5 & 0.2 & 42.0 & 49.5 & 87.6 & $510,735.5$ & $353,265.5$ & $286,250.7$ & $449,185.5$ \\
\hline El Sidrón 1253 & 27.9 & 0.0 & 2.5 & 78.8 & 38.2 & 62.3 & $414,420.4$ & $339,207.2$ & $274,711.4$ & $408,161.1$ \\
\hline
\end{tabular}

859 Table S7.3. Observed branch counts and estimates of TMRCA between archaic and

860 modern human Y chromosomes. All quantities represent averages across a panel of

86113 non-African $Y$ chromosomes (Table S7.1) and are based on A00-based estimates of 862 mutation rate and $T M R C A_{A F R}$ (section 7.1). Counts in columns a to $f$ represent counts of 863 site patterns as shown in Figures S7.4 and S7.5. "Total" represents the number of sites 864 out of the total $6.9 \mathrm{Mb}$ of target sequence available for the analysis. 


\subsection{TMRCA of Mezmaiskaya 2 and Spy $94 a$}

867 The split time of Neandertal and modern human $Y$ chromosomes estimated in the 868 previous section provides an upper bound for the last time the two populations 869 experienced gene flow. Similarly, the deepest divergence in late Neandertal $Y$ 870 chromosomes represents a lower bound, as the introgressed $Y$ chromosome lineage 871 must have already been present in Neandertals prior to this diversification. To estimate the deepest TMRCA of the known Neandertal Y chromosomes (i.e. the TMRCA of Mezmaiskaya 2 and Spy 94a, Figure 2A), we first defined a set of sites in

874 the $\sim 6.9 \mathrm{Mb}$ capture target regions which carry a reference allele in the chimpanzee, A00 875 and French $\mathrm{Y}$ chromosomes (the ancestral state) and an alternative allele (the derived state) on the branch leading to the high-coverage $\mathrm{Y}$ chromosome of Mezmaiskaya 2

877 (Figure S7.14), using the standard filtering used in previous sections (minimum three 878 reads covering each genotyped site, section 5.3). We can calculate the approximate 879 length of this branch using the TMRCA of Mezmaiskaya 2 and modern human $\mathrm{Y}$ 880 chromosomes ( 370 kya, Table S7.3) and the known age of Mezmaiskaya 2 ( 44 kya, 881 (11)) as $370 \mathrm{kya}-44 \mathrm{kya}=326 \mathrm{ky}$ (Figure S7.14). We can then estimate the split time 882 between Mezmaiskaya 2 and Spy 94a Y chromosomes using the proportion of 883 Mezmaiskaya-derived sites which show the ancestral allele in Spy 94a (Figure S7.14). 884 Specifically, if we let $Y$ be the number of ancestral alleles observed in Spy 94a and $X+Y$ 885 be the total number of sites with genotype calls in Spy 94a at positions derived in 886 Mezmaiskaya 2, we can express the TMRCA of $Y$ chromosomes of the two Neandertals 887 simply as

$$
\frac{Y}{X+Y} \times 326+44 \text { kya }
$$


889 (Figure S7.14). We maximized the amount of data available for the analysis by merging 890 the capture data with previously published shotgun sequences (11) and evaluated the 891 robustness of the results to different genotype filtering and classes of polymorphisms. To 892 estimate confidence intervals (C.I.), we re-sampled the $X$ and $Y$ counts from Poisson 893 distributions with expected values given by the observed counts (Figure S7.14), 894 calculated the TMRCA on the re-sampled counts using equation (6) and then took the $8952.5 \%$ and $97.5 \%$ quantiles of this simulated TMRCA distribution to arrive at the 896 approximate range of $95 \%$ C.I.

898 regardless of the filtering criteria used (individual point estimates and $95 \%$ confidence 899 intervals shown in Figure S7.15 and Table S7.4). Together with the TMRCA of Neandertal 900 and modern human $\mathrm{Y}$ chromosomes, this suggests that the gene flow from an early 901 population related to modern humans is likely to have happened some time between $~ 100$ 902 kya and $~ 370$ kya. We note that this time window is significantly wider than the one 903 inferred based on a much more extensive set of available Neandertal mtDNA genomes 904 (219-468 kya) (38). However, it is likely that future sampling of Neandertal Y chromosome 905 diversity will reveal more basal $Y$ chromosome lineages as has been the case for 906 Neandertal mtDNA (38). 


\section{chimpanzee}

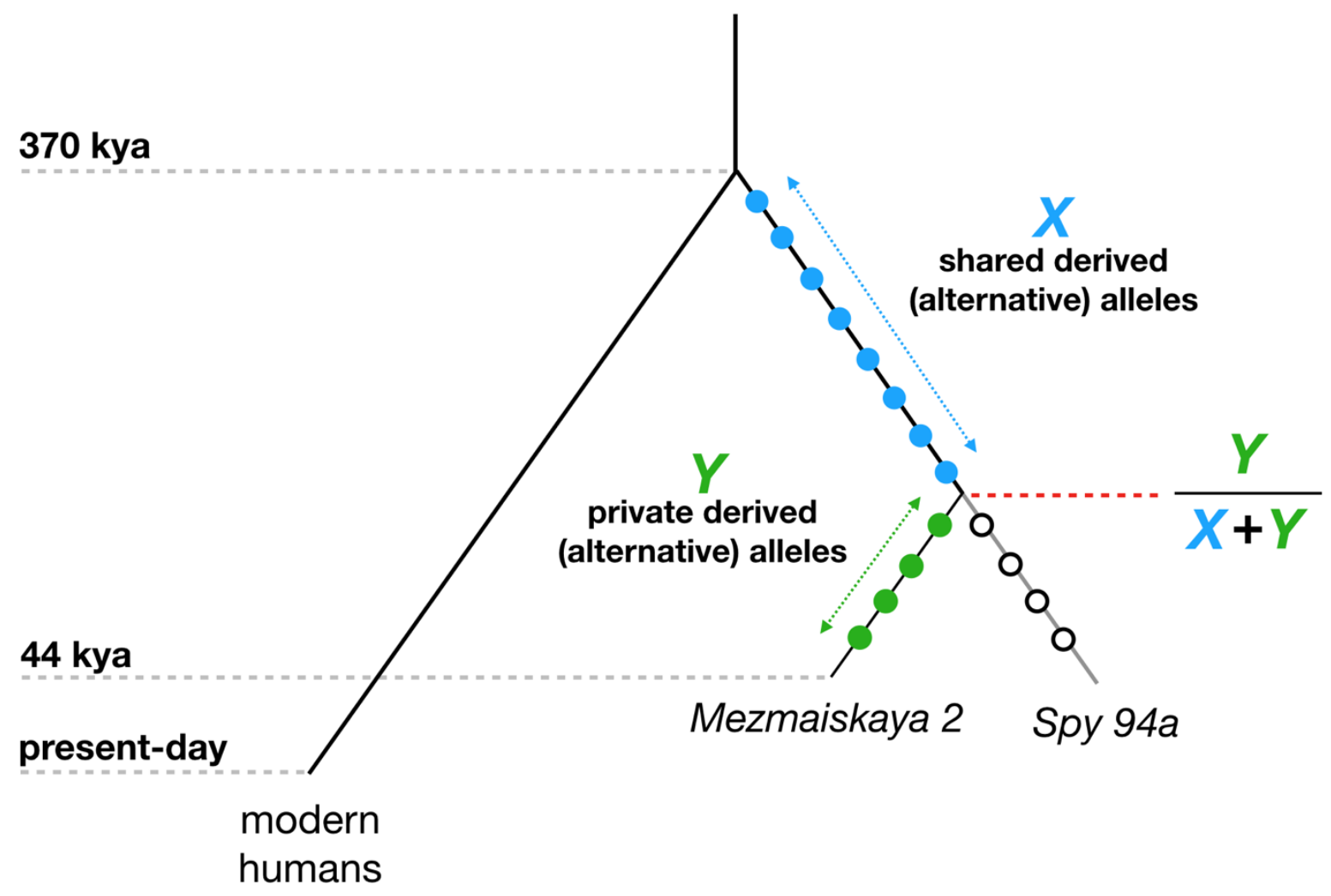

humans

Figure S7.14. Estimating the TMRCA of Neandertal Y chromosomes. Filled circles

911 represent a set of derived (alternative) alleles on the high-coverage Mezmaiskaya 2

912 lineage, and are defined as sites at which Mezmaiskaya $2 \mathrm{Y}$ chromosome carries a

913 different allele than chimpanzee, A00 and French $\mathrm{Y}$ chromosomes. Empty circles

914 represent a subset of such sites which show the ancestral (reference) state in Spy 94a. 


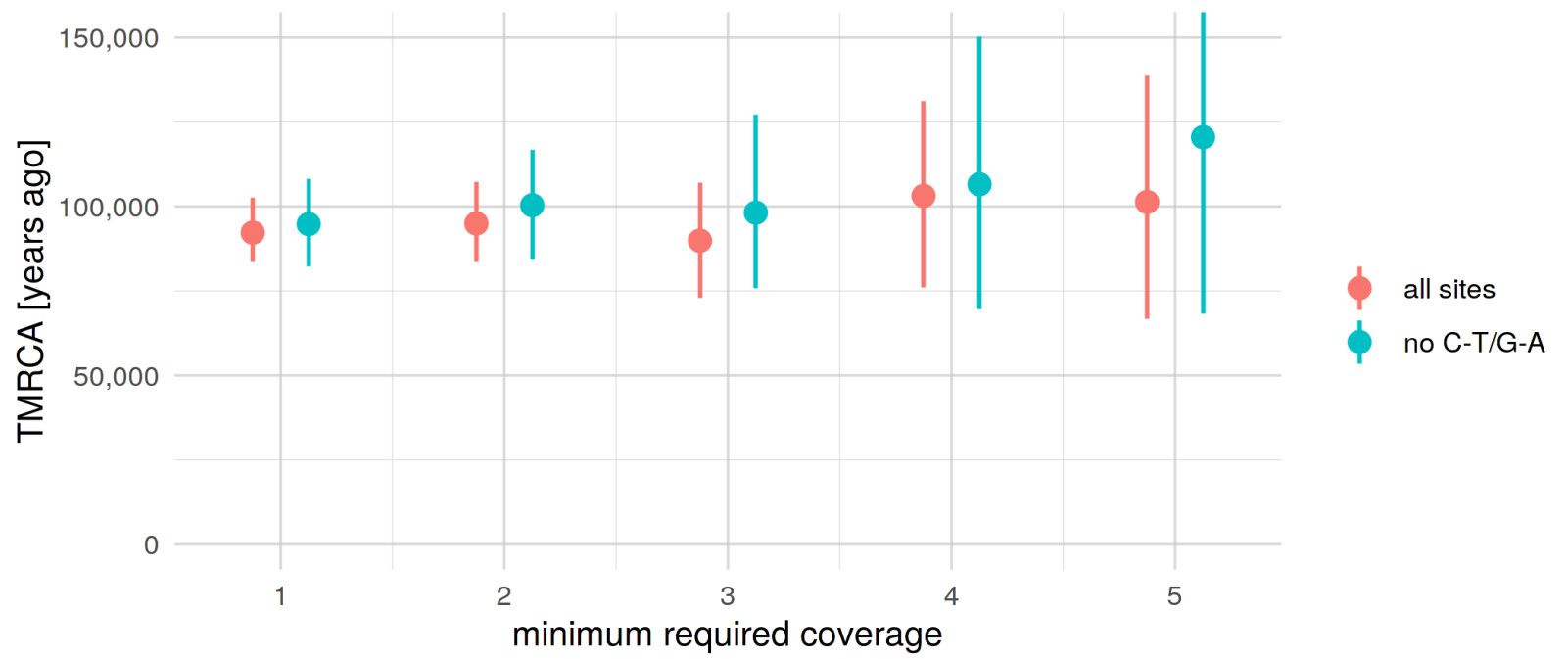

918 Figure S7.15. TMRCA of Mezmaiskaya 2 and Spy 94a. Informative positions (derived

919 alleles in Mezmaiskaya 2) were defined using genotype calls in Mezmaiskaya 2 which

920 passed the standard filtering used throughout our study (minimum coverage of at least

921 three reads, maximum coverage less than $98 \%$ quantile of the total coverage distribution).

922 We called the genotypes in the Spy 94a Y chromosome at these positions and calculated

923 the TMRCA using the equation (6). We tested the robustness of the estimate to genotype

924 calling errors using different minimum coverage filters for Spy 94a (x-axis) and two sets

925 of polymorphisms (colors).

926

927 


\begin{tabular}{|r|r|r|r|r|r|r|r|}
\hline $\begin{array}{r}\text { REF } \\
\text { count }\end{array}$ & $\begin{array}{c}\text { ALT } \\
\text { count }\end{array}$ & $\begin{array}{c}\text { proportion } \\
\text { REF }\end{array}$ & TMRCA & $\begin{array}{c}\text { TMRCA } \\
\text { (lower CI) }\end{array}$ & $\begin{array}{c}\text { TMRCA } \\
\text { (upper Cl) }\end{array}$ & $\begin{array}{c}\text { minimum } \\
\text { coverage }\end{array}$ & calculated on \\
\hline 71 & 469 & 0.1315 & 92250.32 & 83814.88 & 102157.6 & 1 & all sites \\
\hline 51 & 314 & 0.1397 & 94962.33 & 83315.75 & 107309.6 & 2 & all sites \\
\hline 20 & 141 & 0.1242 & 89862.87 & 74453.89 & 108808.4 & 3 & all sites \\
\hline 13 & 66 & 0.1646 & 103130.37 & 77184.06 & 129130.5 & 4 & all sites \\
\hline 7 & 37 & 0.1591 & 101332.33 & 67250.74 & 141779.7 & 1 & all sites \\
\hline 43 & 266 & 0.1392 & 94775.67 & 82515.76 & 108105.1 & 2 & no C-T/G-A \\
\hline 32 & 173 & 0.1561 & 100347.68 & 83703.34 & 117665.3 & 3 & no C-T/G-A \\
\hline 13 & 74 & 0.1494 & 98152.86 & 75463.15 & 125756.6 & 4 & no C-T/G-A \\
\hline 7 & 33 & 0.1750 & 106565.56 & 69559.13 & 149511.3 & 5 & no C-T/G-A \\
\hline 5 & 18 & 0.2174 & 120510.02 & 67229.10 & 180578.4 & 4 & 4 \\
\hline
\end{tabular}




\subsection{Confidence intervals}

937 Under the assumption that mutations on each branch of a tree (Figures S7.1 and S7.4) 938 accumulate independently, the observed counts of mutations can be understood as 939 realizations of independent Poisson processes (mutation counts in Tables S7.2 and 940 S7.3). To quantify the uncertainty in our TMRCA estimates, we used a simulation-based 941 bootstrapping approach. For each set of branch lengths used to calculate scaling factor

$942 \alpha$ (branches $a, d$ and $e$, Table 7.3), we generated 1000 sets of simulated counts by 943 randomly sampling from a Poisson distribution with the parameter $\lambda$ set to values

944 observed from the data. In other words, we simulated "trees" implicitly by generating a 945 set of Poisson-distributed branch lengths. We then used the simulated counts to estimate 946 the corresponding TMRCA values, obtaining a distribution of TMRCA consistent with the

947 observed data. Finally, we took the lower $2.5 \%$ and upper $97.5 \%$ quantiles of the 948 simulated distribution as the boundaries of bootstrap-based $95 \%$ confidence intervals.

949 To estimate the confidence interval for TMRCAs across the whole panel of 13 non950 African Y chromosomes (black dotted horizontal lines in TMRCA figures in our study such 951 as Figure 2B), we followed the same procedure but pooled all simulated counts together 952 (i.e., 1000 simulated counts for each of the $13 \mathrm{Y}$ chromosomes). Then, we took the lower $9532.5 \%$ and upper $97.5 \%$ quantiles of TMRCA estimates calculated from the pooled counts. 


\section{Simulations of introgression under purifying selection}

958 To investigate the expected frequency trajectories of $Y$ chromosomes introgressed from

959 modern humans into Neandertals, we adapted a modeling approach previously used to

960 study negative selection against Neandertal DNA in modern humans $(39,40)$. Briefly, this

961 model assumes lower effective population size $\left(N_{e}\right)$ in Neandertals than modern humans

962 as inferred from comparisons of whole-genome sequences (2). Under nearly-neutral

963 theory, such differences in $N_{e}$ are expected to increase the genetic load in Neandertals

964 compared to modern humans through an excess of accumulated deleterious mutations

965 due to lower efficacy of purifying selection. Therefore, after introgression from

966 Neandertals into modern humans, Neandertal haplotypes would be under stronger

967 negative selection compared to modern humans haplotypes, causing a rapid decrease in

968 proportion of genome-wide Neandertal ancestry $(39,40)$.

969

In the context of evidence for Neandertal Y chromosome replacement in our study,

970 we were particularly interested in the dynamics of introgression in the opposite direction,

971 from modern humans into Neandertals. Specifically, given that nearly-neutral theory

972 predicts that Neandertal $\mathrm{Y}$ chromosomes would carry a higher load of deleterious

973 mutations compared to modern human $\mathrm{Y}$ chromosomes, how much is natural selection

974 expected to favor introgressed modern human $Y$ chromosomes compared to their original

975 Neandertal counterparts?

977 To address this question, we used a forward population genetic simulation framework

978 SLiM (version 3.3) (41) to build an approximate model of modern human and Neandertal

979 demographic histories, following a strategy we used to study the long-term effects of 
980 Neandertal DNA in modern humans (40). To simulate differences in $N_{e}$ of both 981 populations, we set $N_{e}=10,000$ in modern humans and $N_{e}=1,000$ in Neandertals after 982 the split of both lineages from each other at 600,000 years ago. Given the non983 recombining nature of the human $Y$ chromosome, we implemented a simplified model of 984 a genomic structure in which the only parameter of interest is the total amount of 985 sequence under selection ("functional" sequence, ranging from $100 \mathrm{~kb}$ to $2 \mathrm{Mb}$ in steps 986 of $100 \mathrm{~kb}$ ), and set the recombination rate to zero. Furthermore, because the amount of 987 deleterious variation accumulated on both lineages is directly related the time they have 988 been separated from each other, we simulated gene flow from modern humans into 989 Neandertals between 150,000 to 450,000 years ago in steps of 25,000 years (this time 990 range for gene flow encompasses the times inferred by $(38,42,43)$ and our own study), 991 assuming a fixed split time of 600,000 years ago. We ran 100 independent replicates for 992 each combination of parameters described above, including an initial burn-in phase of 99370,000 generations $\left(7 \times\right.$ ancestral $N_{e}$ of 10,000$)$ to let the simulations reach the state of 994 mutation-selection-drift equilibrium.

995 In our previous study, we found evidence for different modes of selection in 996 different classes of functionally important genomic regions, suggesting that the fitness 997 consequences of mutations vary significantly according to the position of their occurrence 998 (40). Realistic modeling of negative selection and introgression would thus require precise 999 information about the distributions of fitness effects (DFE), dominance and epistasis for 1000 coding, non-coding and regulatory regions. Unfortunately, with the exception of DFE of 1001 amino acid changing de novo mutations affecting autosomal genes $(44,45)$, little is known 1002 about fitness consequences of non-coding and regulatory mutations on the $Y$ 
1003 chromosome. Furthermore, the impact of $\mathrm{Y}$ chromosome structural variation in the 1004 context of male fertility is highly significant, but still relatively poorly understood in terms 1005 of its DFE $(46,47)$. These issues, as well as the large parameter space of all relevant 1006 demographic and selection factors make analyzing the model dynamics quite challenging.

1007 To make our results easier to interpret, we scored each simulation run (i.e., each 1008 introgression frequency trajectory) with the ratio of fitness values of the average 1009 Neandertal $\mathrm{Y}$ chromosome and the average modern human $\mathrm{Y}$ chromosome generated 1010 by the simulation, just prior to the introgression. This way we collapse many potential 1011 parameters into a single relevant measure (how much worse are Neandertal Y 1012 chromosomes compared to modern human $\mathrm{Y}$ chromosomes in terms of evolutionary 1013 fitness) while, at the same time, generalizing our conclusions to other potential factors we 1014 do not model explicitly.

1015 To calculate the fitness of simulated $Y$ chromosomes, we used the fact that 1016 mutations in SLiM behave multiplicatively, i.e. each mutation affects any individual's 1017 fitness independently of other mutations. Following basic population genetics theory (48), 1018 if we let the fitness of an individual be $W$ and the fitness of each mutation be $w_{i}$, we can 1019 define $W$ as $W=\prod w_{i}=\prod\left(1-s_{i}\right)$, where $i$ runs across all mutations carried by this 1020 individual and $s_{i}$ is the selection coefficient of the $i$-th deleterious mutation. We can then 1021 transform multiplicative interaction into log-additive interaction by

$$
W=\prod w_{i}=e^{\sum \log w_{i}}=e^{\sum \log \left(1-s_{i}\right)} \approx e^{\sum\left(-s_{i}+\frac{s_{i}^{2}}{2}\right)} \approx e^{-\sum s_{i}}
$$

1023 using simple rules of Taylor expansion under the assumption that we are dealing with 1024 weakly deleterious mutations whose selection coefficients $\left(s_{i}\right)$ are expected to be very 1025 small (48). 
1027 In practice, we let each simulation replicate run until modern human introgression into 1028 Neandertals, at which point we saved all Neandertal and modern human Y chromosomes 1029 and their mutations to a SLiM population output file. After introgression, which we 1030 simulated at $5 \%$, we tracked the frequency of modern human $\mathrm{Y}$ chromosomes in 1031 Neandertals for 100,000 years, saving the frequency values at regular time intervals to 1032 another output file.

For the downstream analyses, we calculated the fitnesses of all Neandertal and 1034 modern human $\mathrm{Y}$ chromosomes from the population output files saved in the first step 1035 using equation (7) and calculated the ratio of the mean fitness values in both populations 1036 - this measure quantifies the expected decrease in fitness of Neandertal Y chromosomes 1037 compared to modern human $\mathrm{Y}$ chromosomes. The distribution of simulated fitness ratios 1038 across a two-dimensional parameter grid is shown in Figure S8.1. As expected, longer 1039 times of separation between Neandertals and modern humans and larger amounts of 1040 mutational target sequence increases the average genetic load of Neandertals. To 1041 analyze the dynamics of introgression in Neandertals over time, we scored the simulated 1042 modern human $\mathrm{Y}$ chromosome frequency trajectories with the calculated fitness decrease 1043 obtained from the simulation (Figure S8.2). Finally, we estimated the expected probability 1044 of replacement of the original Neandertal $Y$ chromosomes over time by counting the 1045 proportion of the simulated trajectories (over all trajectories in each fitness bin) in which 1046 the introgressed modern human $\mathrm{Y}$ chromosomes reached fixation in each time point 1047 (Figure S8.2). These trajectories of replacement probabilities are shown in Figure 3B. 
1048 Similarly to Figure S8.1, Figure S8.3 shows these probabilities expanded from the single 1049 fitness reduction score back on the two-dimensional parameter grid.

1051 chromosomes, the conclusions based on the abstract measure of fitness reduction can

1052 be generalized even to the case of mtDNA introgression (Figure 3B). 


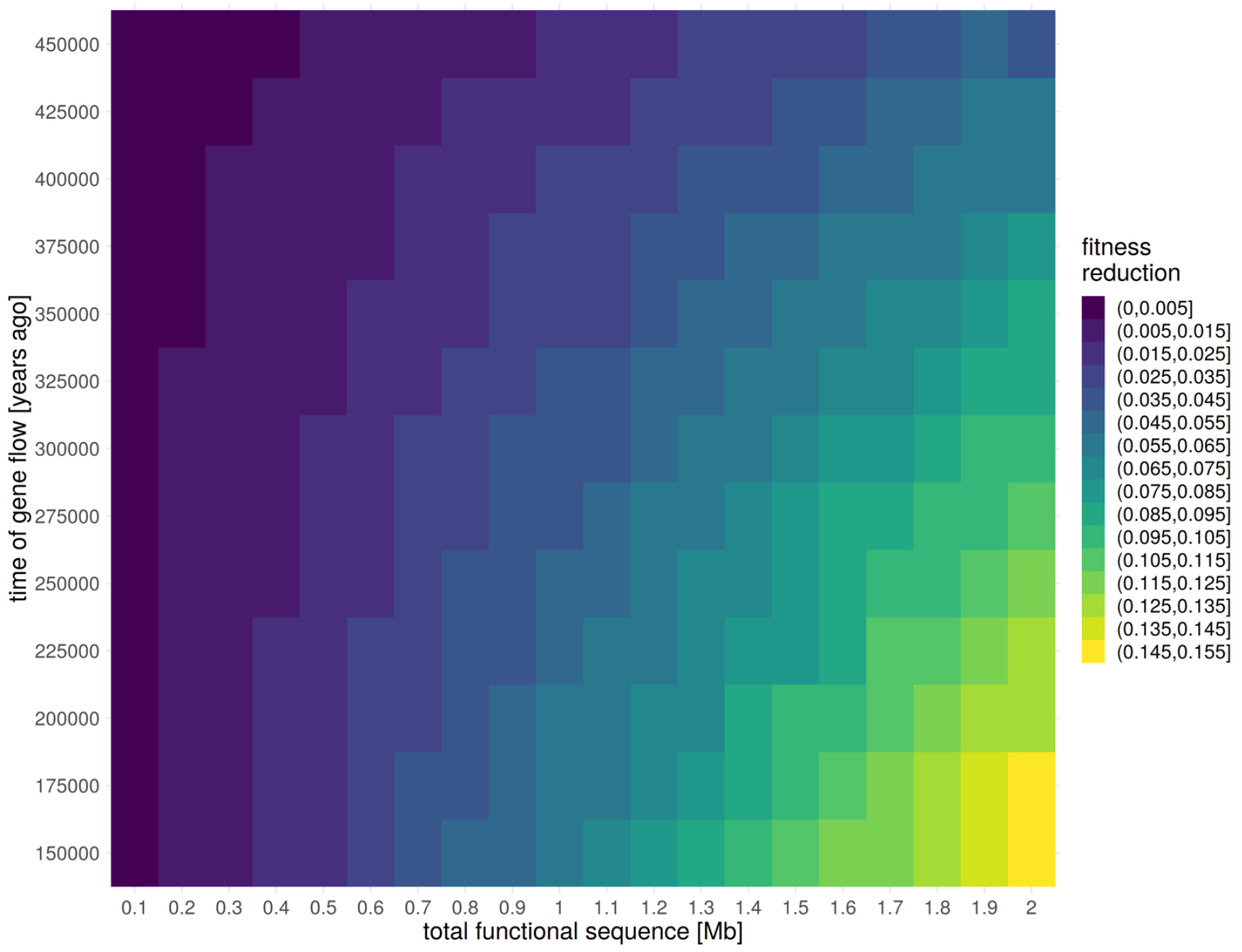

Figure S8.1. Expected decrease in fitness of an average Neandertal Y chromosome were averaged over 100 independent simulation replicates on a grid of two parameters as the ratios of the mean fitness of Neandertal $Y$ chromosomes to the mean fitness of 1060 modern human $Y$ chromosomes (calculated using equation 7). Lighter colors represent 1061 lower fitness of Neandertal $Y$ chromosomes compared to modern human $Y$ 1062 chromosomes. 


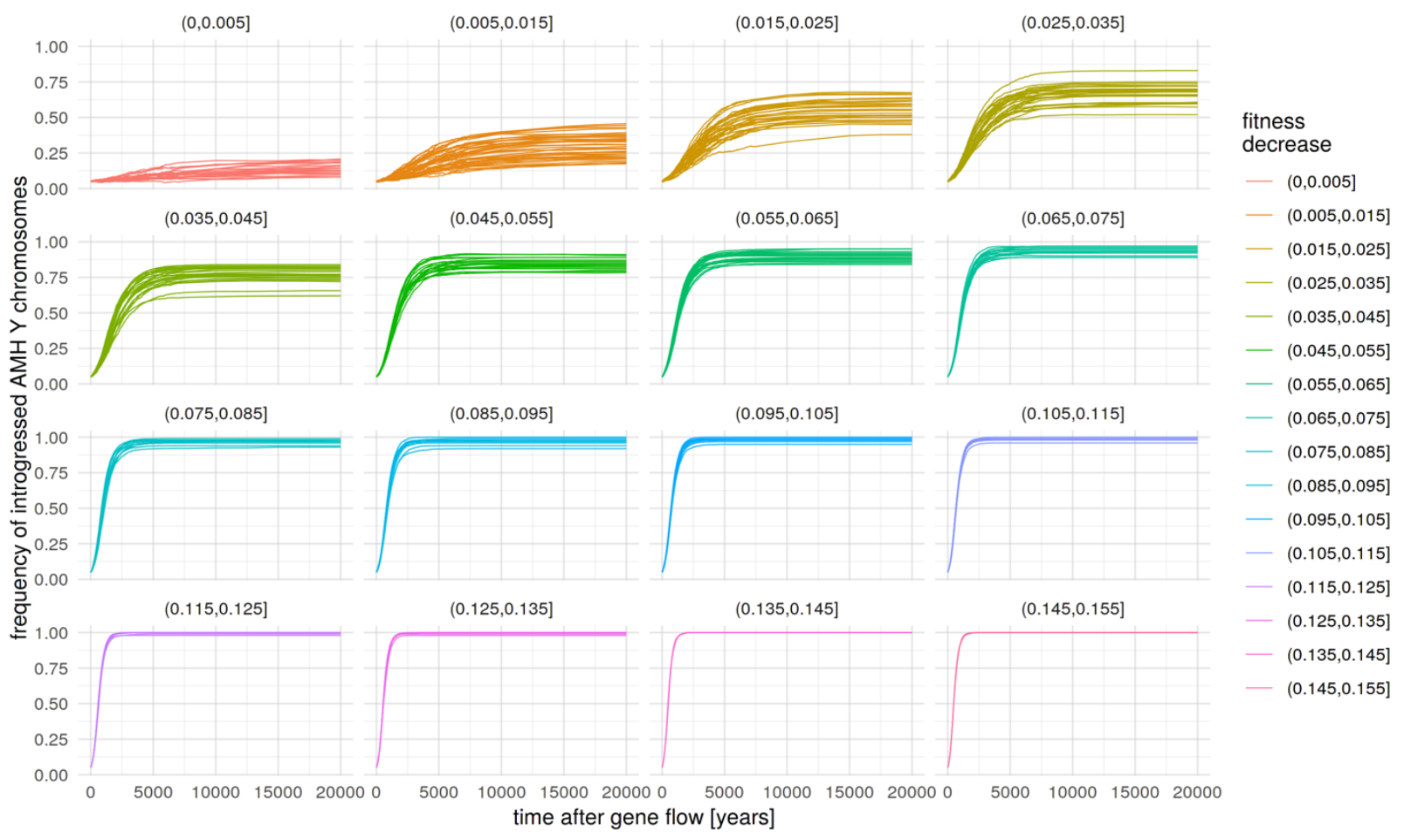

Figure S8.2. Frequency trajectories of introgressed modern human $Y$

1067 chromosomes in Neandertals, partitioned by the fitness decrease of Neandertal $\mathbf{Y}$ 1068 chromosomes compared to modern human Y chromosomes. 


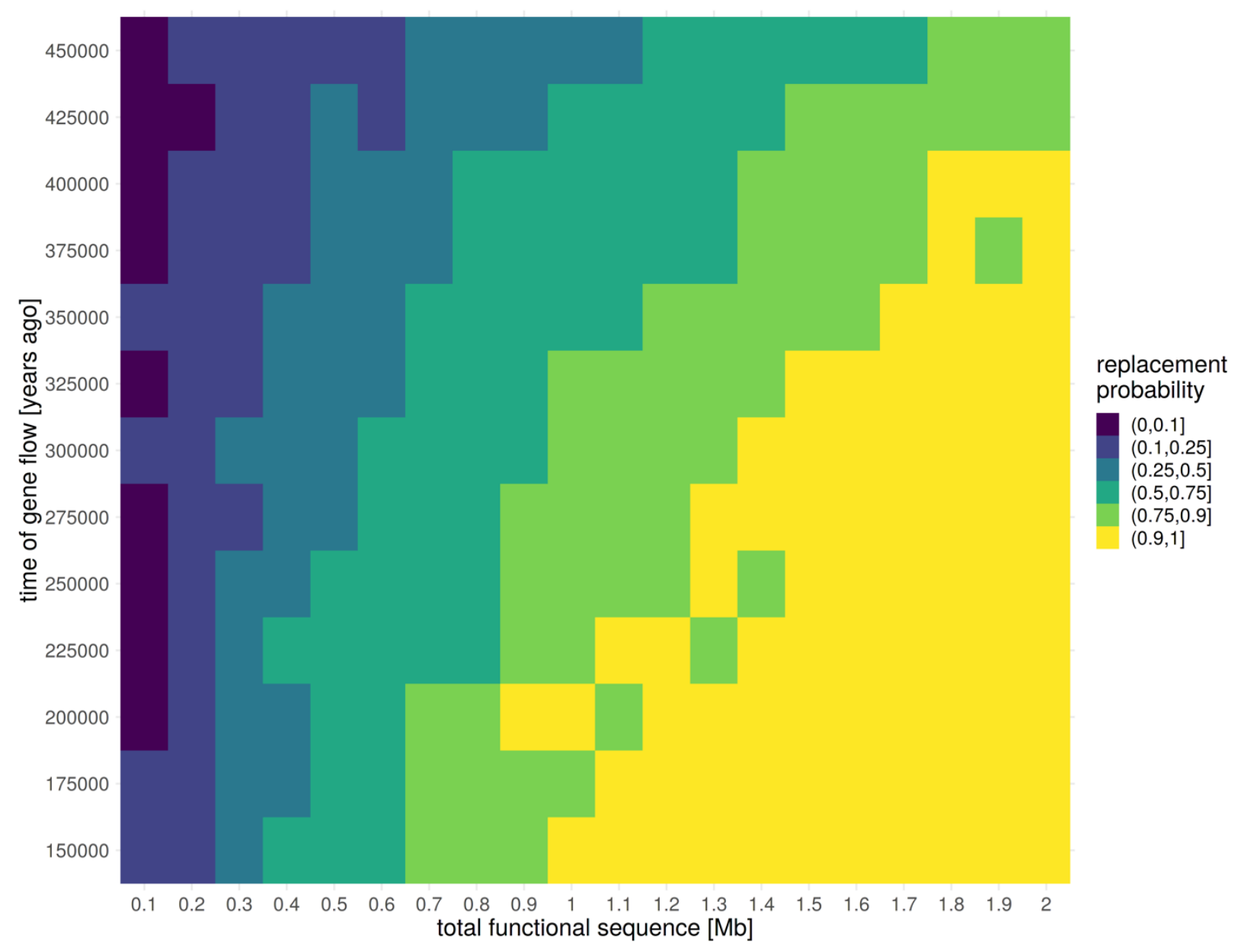

1073 Figure S8.3. Probability of replacement of a Neandertal $Y$ chromosome at 20 1074 thousand years after gene flow from modern human. Probabilities represent the 1075 proportion of introgressed modern human $\mathrm{Y}$ chromosome trajectories that reached 1076 fixation in the Neandertals after 20 thousand years after gene flow, out of the total 100 1077 simulation replicates performed for each combination of two-dimensional parameters 1078 (Figure S8.2). 
1. G. Benson, Nucleic Acids Res 27, (1999).

2. K. Prüfer et al., Nature 505, 43 (2014).

1084

3. Q. Fu et al., Proceedings of the National Academy of Sciences 110, 2223 (2013).

4. S. Castellano et al., Proceedings of the National Academy of Sciences 111, 6666 (2014).

1088 6. S. Lippold et al., Investig Genet 5, 13 (2014).

1089 7. J. Dabney et al., Proceedings of the National Academy of Sciences 110, 15758 (2013).

1090 8. P. Korlević et al., BioTechniques 59, (2015).

1091 9. I. Glocke, M. Meyer, Genome Res 27, 1230 (2017).

1092 10. N. Rohland, I. Glocke, A. Aximu-Petri, M. Meyer, Nat Protoc 13, 2447 (2018).

1093 11. M. Hajdinjak et al., Nature 555, 652 (2018).

1094

1095

M. T. Gansauge, M. Meyer, Nat Protoc 8, 737 (2013).

1096

1097

1098

1099

13. M.-T. Gansauge et al., Nucleic Acids Research gkx033 (2017).

14. V. Slon et al., Sci Adv 3, e1700186 (2017).

15. M. Kircher, S. Sawyer, M. Meyer, Nucleic Acids Res 40, e3 (2012).

16. J. Dabney, M. Meyer, BioTechniques 52, (2012).

17. G. Renaud, U. Stenzel, J. Kelso, Nucleic Acids Research 42, e141 (2014).

18. H. Li, R. Durbin, Bioinformatics 25, 1754 (2009).

1101

19. H. Li et al., Bioinformatics 25, 2078 (2009).

1102

1103

20. S. Mallick et al., Nature 538, 201 (2016).

M. Karmin et al., Genome Research 25, 459 (2015).

1105

22. Q. Fu et al., Nature 514, 445 (2014).

1106

1107

23. F. L. Mendez et al., Am J Hum Genet 92, 454 (2013).

24. A. R. Quinlan, I. M. Hall, Bioinformatics 26, 841 (2010).

1108

25. A. W. Briggs et al., Proceedings of the National Academy of Sciences 104, 14616 (2007).

26. T. Günther, C. Nettelblad, PLoS Genet 15, e1008302 (2019).

27. H. Li, Bioinformatics 27, 2987 (2011).

28. K. Prüfer, Bioinformatics 34, 4165 (2018).

29. T. Kivisild, Hum Genet 136, 529 (2017).

30. K. P. Schliep, Bioinformatics (2011).

31. E. Paradis, K. Schliep, Bioinformatics (2019).

32. M. K. Kuhner, J. Felsenstein, Molecular biology and evolution 11, 459 (1994).

33. G. Yu, D. K. Smith, H. Zhu, Y. Guan, T. T.-Y. Lam, Methods in Ecology and Evolution 8, 28 (2017).

34. K. Douka et al., Nature 565, 640 (2019).

35. P. Hallast et al., Molecular Biology and Evolution 32, 661 (2015).

1120

36. C. Barbieri et al., Human Genetics 135, 541 (2016).

37. P. Hallast et al., Genome Research 26, 427 (2016).

38. C. Posth et al., Nat Commun 8, 16046 (2017).

39. K. Harris, R. Nielsen, Genetics 203, 881 (2016).

40. M. Petr, S. Pääbo, J. Kelso, B. Vernot, Proc Natl Acad Sci U S A 116, 1639 (2019).

41. B. C. Haller, P. W. Messer, Molecular biology and evolution 36, 632 (2019).

42. M. J. Hubisz, A. L. Williams, A. Siepel, (2019).

43. L. Chen, A. B. Wolf, W. Fu, L. Li, J. M. Akey, Cell 180, 677 (2020). 
1127 44. A. Eyre-Walker, M. Woolfit, T. Phelps, Genetics 173, 891 (2006).

1128 45. A. R. Boyko et al., PLoS Genet 4, e1000083 (2008).

1129 46. W. A. Brashear, T. Raudsepp, W. J. Murphy, Genome Res 28, 1841 (2018).

1130 47. S. Colaco, D. Modi, Reproductive biology and endocrinology 16, 14 (2018).

1131

48. J. F. Crow, M. Kimura, An Introduction to Population Genetics Theory 1970), pp. 591. 


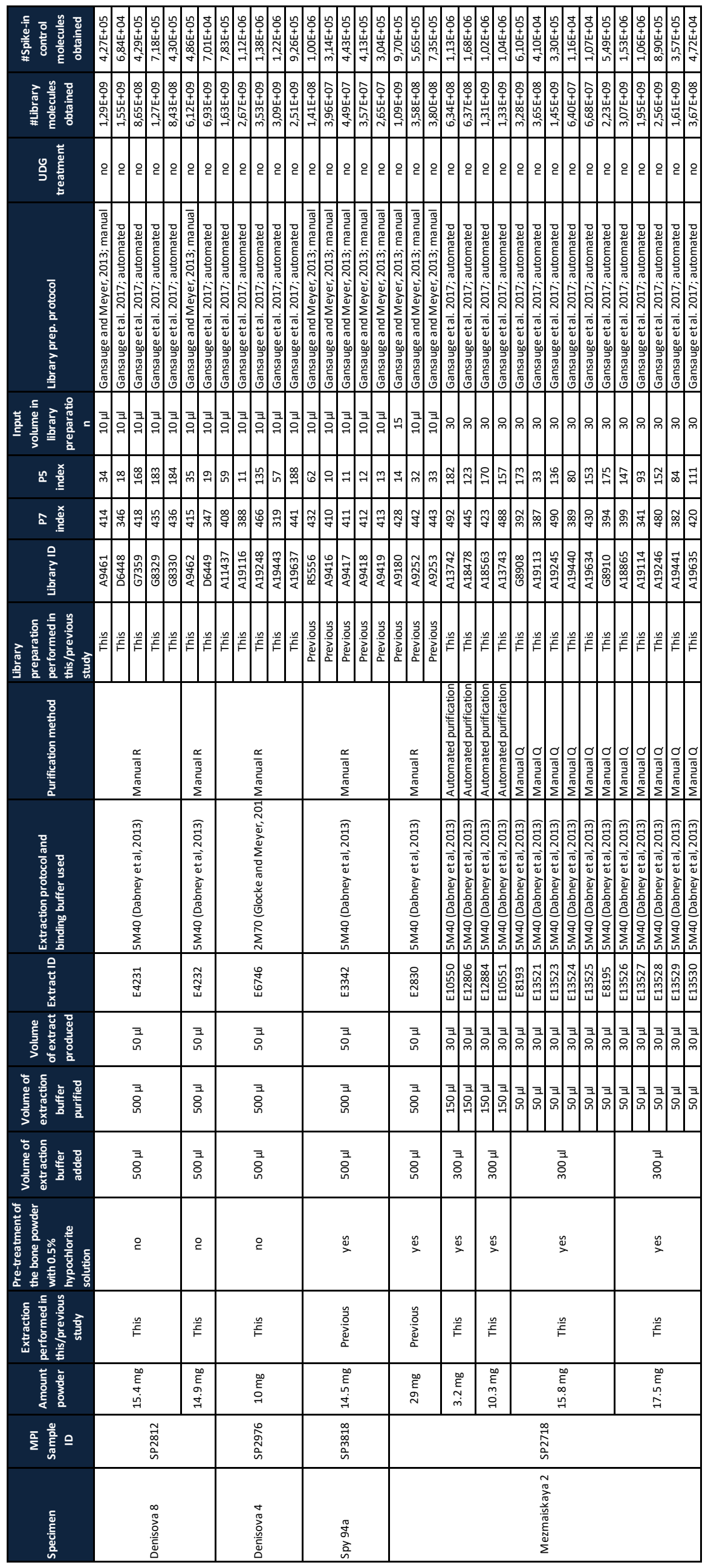

